\begin{abstract}
TADINADA, SASHI KANTH. Consideration of Uncertainties in Seismic Analysis of Non-classically Damped Coupled Systems. (Under the Direction of Dr. Abhinav Gupta and Dr. Vernon Matzen.)

The objective of this study is to investigate the effect of uncertainties in modal properties of uncoupled primary and secondary systems in the seismic analysis of non-classically damped Multi Degree of Freedom (MDOF) primary - MDOF secondary coupled systems by response spectrum method.
\end{abstract}

The design response of the secondary system is evaluated by conducting multiple analyses of coupled system with randomly sampled sets of uncoupled natural frequencies of primary and secondary systems. Generating the random samples of frequencies require knowledge of their probability density functions. When the individual probability density functions overlap, generating sample sets by assuming the frequencies to be independent random variables can lead to incorrect sets of frequencies in the sense that the frequencies do not remain as ordered pairs or ordered sets. The frequencies of any uncoupled primary or secondary system should all be in an ascending order. This necessitates the need for considering correlations between such closely spaced frequencies. However, determination of correlations between the natural frequencies of a structure is either not possible or impractical. So, any sampling scheme should consider the constraint that any randomly sampled set of uncoupled frequencies be an ordered set. Moreover, rejecting the incorrect samples result in individual density functions that are significantly different from the distributions initially assumed for sampling of each natural frequency. A formulation for a joint probability density function for the frequencies is developed using fundamental 
probability approaches. Sampling the frequencies by using the joint density function ensures that each sampled set remains ordered while maintaining the individual density functions.

Development of formulation for the joint density function of frequencies enabled the application of the Square-root-of-mean-of-squares (SRMS) method proposed by Gupta and Choi (2005) to MDOF primary - MDOF secondary systems. Two types of MDOF primary MDOF secondary system configurations considered in this validation and verification study are (i) singly-connected secondary systems and (ii) multiply-connected secondary systems. Various degrees of tuning between primary and secondary systems were considered. The modified SRMS method is validated for both types of configurations. 


\title{
Consideration of Uncertainties in Seismic Analysis of Non-Classically Damped Coupled Systems
}

\author{
by \\ Sashi Kanth Tadinada \\ A thesis submitted to the Graduate Faculty of \\ North Carolina State University \\ in partial fulfillment of the \\ requirements for the Degree of \\ Master of Science
}

Civil Engineering

Raleigh, North Carolina

2009

APPROVED BY:

Dr. Abhinav Gupta

Chair of Advisory Committee
Dr. Vernon C. Matzen

Co-Chair of Advisory Committee

Dr. James M. Nau 


\section{BIOGRAPHY}

Sashi Kanth Tadinada was born on August $26^{\text {th }}, 1983$ in Kakinada, India. His family resides in Rajahmundry, India. He joined the undergraduate program in civil engineering at Indian Institute of Technology, Roorkee in July 2000 and received the Bachelors of Technology (B.Tech) degree in May 2004. Following graduation, he worked for two years at Larsen \& Toubro Ltd., - Engineering Construction and Contracts Division (L\&T-ECCD) as a senior engineer in their Transportation Infrastructure (TI) Business Unit in New Delhi, India. In August 2006, he came to U.S. to join the graduate school at North Carolina State University. He graduated with a Master of Science in Civil Engineering in Fall 2008. He is currently pursuing doctoral program in civil engineering at NCSU. 


\section{ACKNOWLEDGEMENTS}

I would like to thank my advisor, Dr. Abhinav Gupta for giving me the opportunity to pursue my graduate studies and perform research at North Carolina State University. His constant guidance and support throughout my research as well as in the preparation of this manuscript has been invaluable to me. I would like to thank Dr. Vernon Matzen and Dr. James Nau for serving on my committee and for their valuable suggestions.

I especially thank Naatu (Harish), Roopa and Isaac for time and patience to discuss my research and their helpful comments. I would like to express my gratitude to all my friends at NCSU for their wonderful company during my stay here.

Lastly, none of this would have been possible without the unconditional love, support and encouragement from my parents and my brother. I appreciate the sacrifices they made for me to have this education. 


\section{TABLE OF CONTENTS}

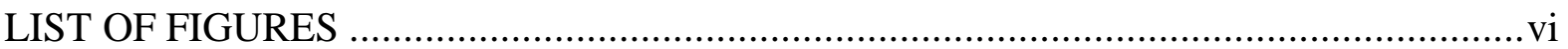

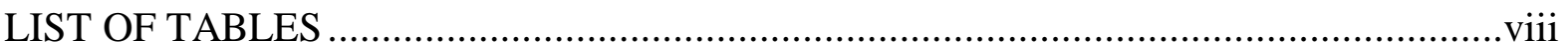

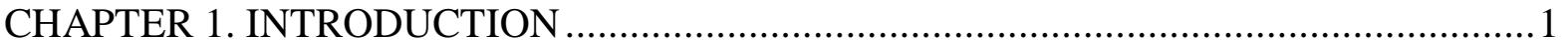

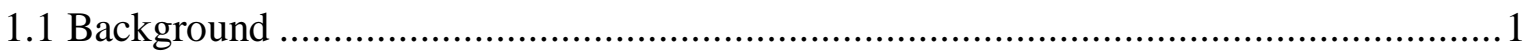

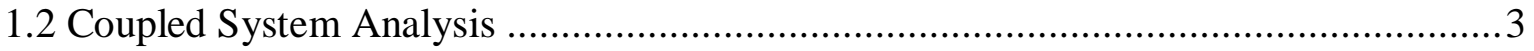

1.3 Incorporation of Uncertainties in Primary-Secondary System Analysis .....................8

1.3.1 Peak Broadening \& Peak Shifting ......................................................... 9

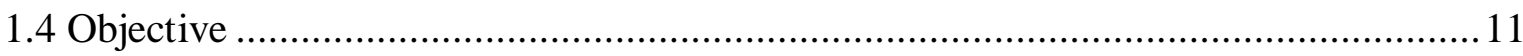

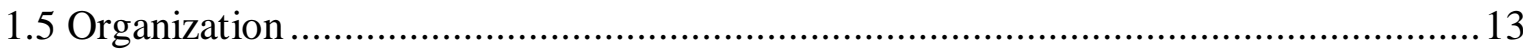

2. MODELING UNCERTAINTY IN MODAL PROPERTIES FOR MDOF SYSTEMS .... 15

2.1 Existing Studies in Evaluation of Eigenproperties' Statistics ...............................20

2.2 Necessary Constraints for Sampling of Natural Frequencies................................26

2.2.1 Problem in Rejection of Disordered Samples ..............................................2 27

\section{JOINT PROBABILITY DENSITY FUNCTION OF NATURAL FREQUENCIES OF A}

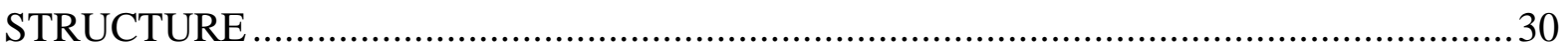

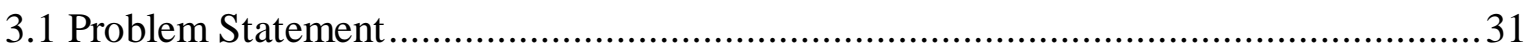

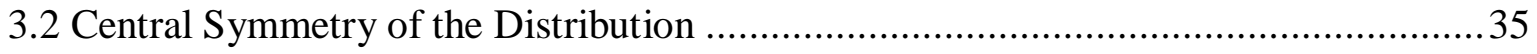

3.3 Derivation of the Bi-variate Joint Probability Density Function .............................38

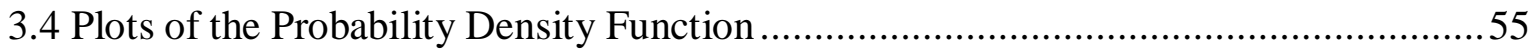

3.5 Sampling of Frequencies from Inverse CDF Method.......................................63 
3.6 Illustrative Example for Sampling of Frequencies

4. SQUARE ROOT OF MEAN OF SQUARES METHOD OF DETERMINING DESIGN

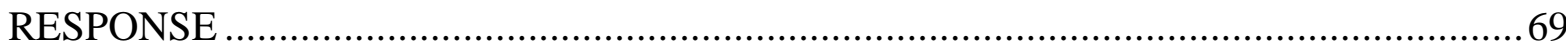

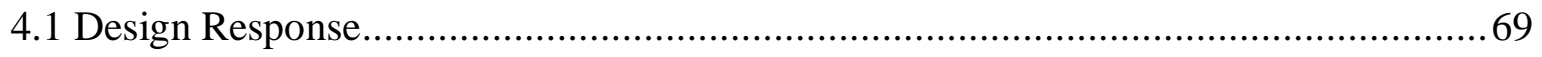

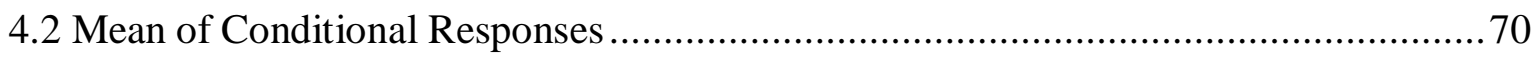

4.3 Square Root of Mean of Squares (SRMS) .............................................................. 71

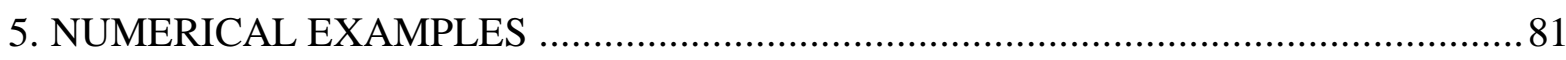

5.1 Relative Significance of Uncertainties in Earthquake Input and Modal Properties .......81

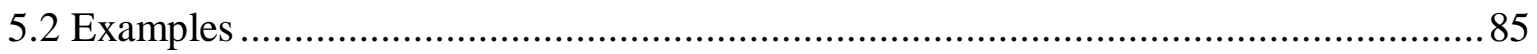

6. SUMMARY, CONCLUSIONS AND FUTURE WORK …………….......................... 102

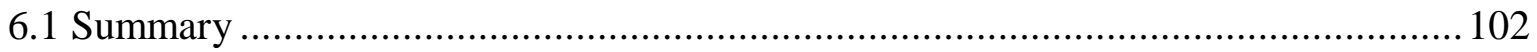

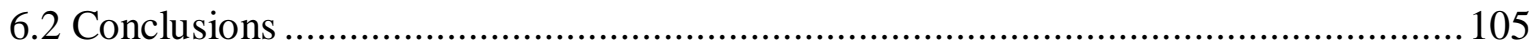

6.3 Limitations and Recommendations for Future Work ……........................................ 107

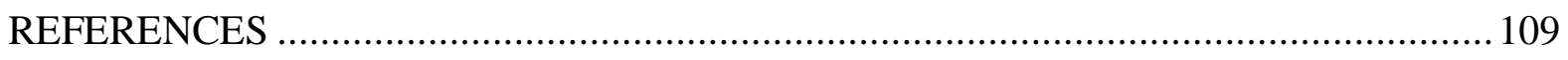

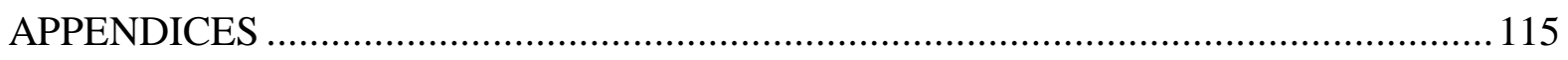

APPENDIX -A: PROBABILITY DENSITY FUNCTIONS OF NATURAL FREQUENCIES OF A SIMPLY SUPPORTED BEAM WITH SHEAR DEFORMATIONS .......................116

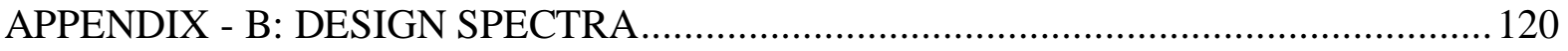




\section{LIST OF FIGURES}

Figure 2.1: Probability density function of first 10 natural frequencies of a beam

Figure 2.2: A 4-DOF system considered to evaluate the correlations between the natural

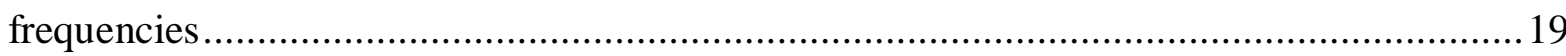

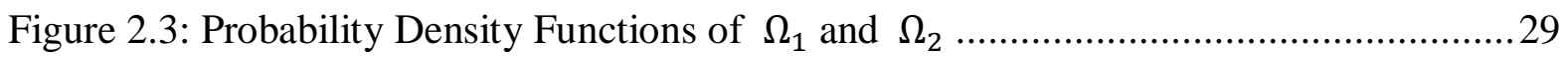

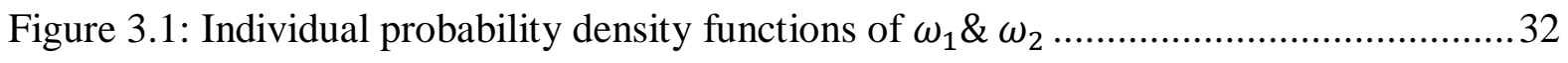

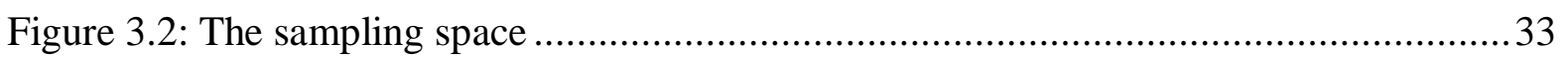

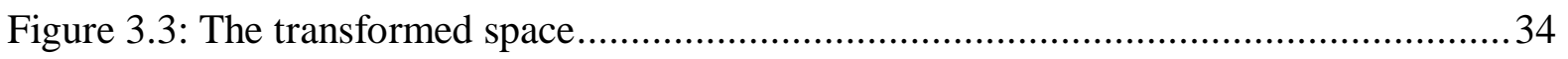

Figure 3.4: Illustration of Central symmetry in the joint density ...................................37

Figure 3.5: Effective sample space due to central symmetry of the density .......................37

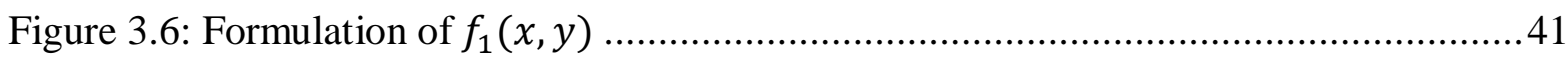

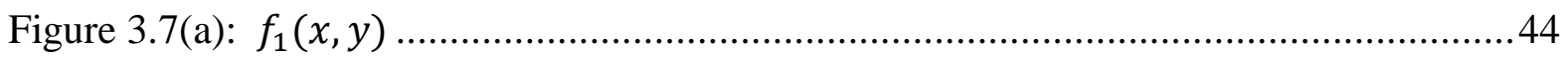

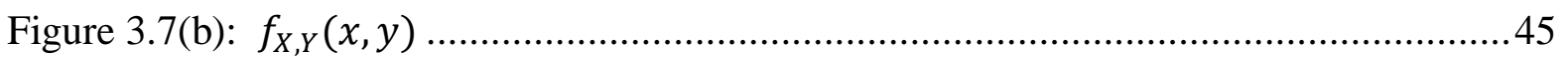

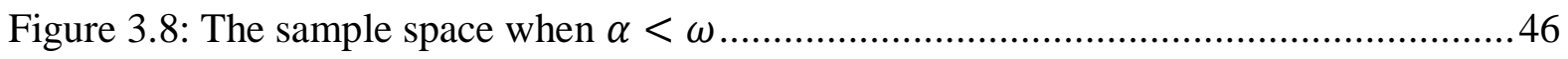

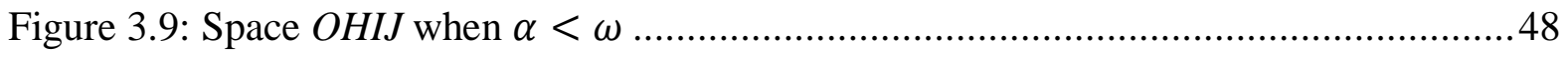

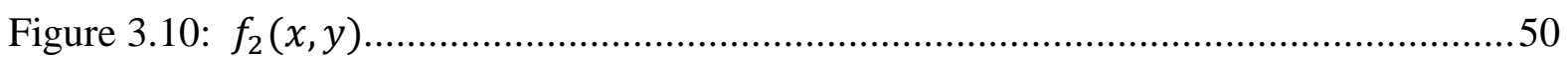

Figure 3.11: $f_{X, Y}(x, y)$ in space $O H I J$ showing various layers when $\alpha<\omega \ldots \ldots \ldots \ldots \ldots \ldots . . .51$

Figure 3.12(a): $f_{\Omega_{1}, \Omega_{2}}\left(\omega_{1}, \omega_{2}\right)$ for case 2 (view of the $\omega_{1}-\omega_{2}$ plane) .........................57

Figure 3.12(b): $f_{\Omega_{1}, \Omega_{2}}\left(\omega_{1}, \omega_{2}\right)$ for case 2 (perspective view) .....................................58

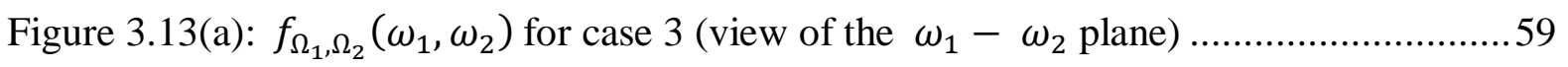

Figure 3.13(b): $f_{\Omega_{1}, \Omega_{2}}\left(\omega_{1}, \omega_{2}\right)$ for case 3 (perspective view) ....................................60 
Figure 3.14(a): $f_{\Omega_{1}, \Omega_{2}}\left(\omega_{1}, \omega_{2}\right)$ for case 4 (view of the $\omega_{1}-\omega_{2}$ plane) .........................61

Figure 3.14(b): $f_{\Omega_{1}, \Omega_{2}}\left(\omega_{1}, \omega_{2}\right)$ for case 4 (perspective view up to 2 layers only) ..............62

Figure 3.15: Probability density function of sampled $\omega_{2}$ (Case 4). 67Figure 3.16: Comparison between Empirical CDF and the expected CDF for $\Omega_{2}$ (Case 4) .....................................68

Figure 5.1: The 2 DOF primary - 2 DOF secondary system model............................... 82

Figure 5.2: Relative significance of uncertainties in earthquake input and modal properties on

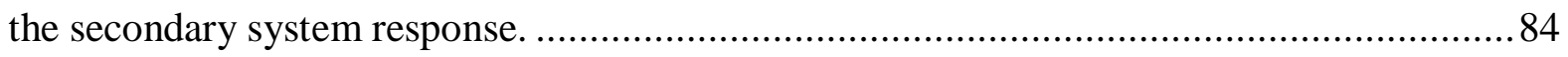

Figure 5.3: Probability density function of sampled values of frequencies of the secondary

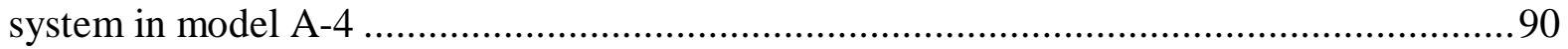

Figure 5.4: Secondary system design responses for model A-1 (Example - A)..................91

Figure 5.5: Secondary system design responses for model A-2 (Example - A)..................91

Figure 5.6: Secondary system design responses for model A-3 (Example - A)..................92

Figure 5.7: Secondary system design responses for model A-4 (Example - A)..................92

Figure 5.8: Secondary system design responses for model A-5 (Example - A)..................93

Figure 5.9: The 8 DOF primary - 3 DOF secondary system structure for model B-1 ..........96

Figure 5.10: The 8 DOF primary - 3 DOF secondary structure for model B-2 .................97

Figure 5.11: Probability density functions of sampled uncoupled frequencies of primary

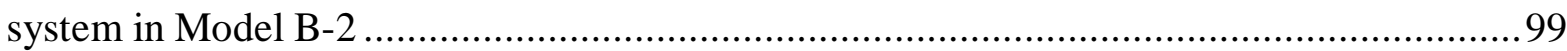

Figure 5.12: Secondary system design responses for model B-1 (Example - B) ............... 100 Figure B.1: Input spectrum corresponding to a non-exceedence probability of 0.84 for 75 real

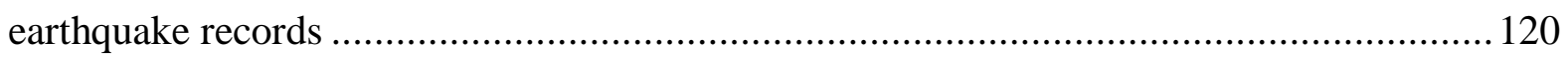




\section{LIST OF TABLES}

Table 3.1: Number of layers in $f_{X, Y}(x, y)$ as a function of mean frequency ratio................53

Table 3.2: Different cases considered for plots of the joint probability density function.......55

Table 5.2: Properties of uncoupled primary and secondary systems in Example - A ...........89

Table 5.3: Mean values of natural frequencies for uncoupled primary and secondary systems

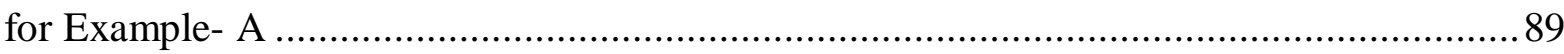

Table 5.4: Comparison between the values of design member forces in the secondary system

obtained from time history input and SRMS method (Example - A) ............................94

Table 5.5: Modal properties of uncoupled primary and secondary systems in Example - B..98

Table B.1: List of real earthquake records 121 


\section{CHAPTER - 1 \\ INTRODUCTION}

\subsection{Background}

The operation of any nuclear power plant relies on a variety of secondary systems such as pipelines, mechanical and electrical equipment which are supported on the primary systems such as the buildings. The importance of secondary systems in seismic performance of a nuclear power plant is now well recognized by researchers and practicing engineers. It is clear that non-performance of the secondary systems during an earthquake can result in major economic losses as well as pose real threat to the safety and integrity of a nuclear plant.

Secondary system response, in addition to its own dynamic properties also depends on the primary system it is supported on. Tuning between primary and secondary system modes can significantly affect the response of a secondary system. Also, the damping characteristics of the primary and secondary systems are generally different, thus making the system non-classically damped. Uncertainty in frequencies can cause the modes of uncoupled

primary and secondary systems to be tuned or detuned. Therefore, incorporation of these uncertainties during the seismic analysis of these structural systems is essential. 
It is non-pragmatic to perform dynamic analysis of the coupled system by treating it as a single large scale model. Large differences in stiffnesses and mass properties between primary and secondary systems can cause numerical instability. As the secondary systems are light, conventional methods commonly assume that they do not interact with their supporting structure - an assumption that the primary and secondary structures can be 'decoupled' for analysis purposes. In these methods, the seismic analysis of primary and secondary systems is done separately. The primary system is analyzed for the input ground motion to obtain the support excitations (floor time histories or spectra) that define the input to the secondary system from which its response is evaluated.

The above approach is reasonable if the secondary structure is very light and the dynamic interaction effect is negligible. The effect of mass interaction and non-classical damping becomes more prominent when the decoupled systems are tuned or nearly tuned (Gupta and Jaw, 1986; Gupta, 1992). Also, multiply-connected secondary systems require consideration of spatial coupling (cross-correlations between support excitations). To account for the effects of mass interaction and non-classical damping, a coupled analysis can be performed by modal synthesis (Gupta, 1992; Igusa and Der Kiureghian, 1992; Gupta and Gupta, 1998). The coupled analysis requires only the input spectrum at the base of the primary system and thus eliminates the need for generating floor spectra at support locations. Also, the responses evaluated from such a coupled analysis are shown to be significantly 
lower than those calculated from a conventional uncoupled analysis (Gupta and Gupta 1995; Gupta and Aradhya, 1996).

\subsection{Coupled System Analysis}

The equation of motion for an $N$-DOF coupled primary - secondary system is given by:

$$
\boldsymbol{M} \ddot{\boldsymbol{U}}+\boldsymbol{C} \dot{\boldsymbol{U}}+\boldsymbol{K} \boldsymbol{U}=-\boldsymbol{M} \cdot \boldsymbol{U}_{\boldsymbol{b}} \cdot \ddot{u}_{g}
$$

where $\boldsymbol{M}, \boldsymbol{C}$ and $\boldsymbol{K}$ are the mass, damping and stiffness matrices of the coupled system; $\boldsymbol{U}, \dot{\boldsymbol{U}}, \ddot{\boldsymbol{U}}$ are the displacement, velocity and acceleration vectors of the coupled system relative to the fixed base of the primary system; $\boldsymbol{U}_{\boldsymbol{b}}$ the static displacement vector of the coupled system and $\ddot{u_{g}}$ is the ground acceleration.

The uncoupled mode shapes $[\Phi]$ are given by

$$
\begin{gathered}
{[\boldsymbol{\Phi}]=\left[\begin{array}{cc}
\boldsymbol{\Phi}_{p} & 0 \\
0 & \boldsymbol{\Phi}_{s}
\end{array}\right]} \\
\boldsymbol{\Phi}_{p}=\left[\boldsymbol{\Phi}_{p 1} \boldsymbol{\Phi}_{p 2} \ldots\right] ; \boldsymbol{\Phi}_{s}=\left[\boldsymbol{\Phi}_{s 1} \boldsymbol{\Phi}_{s 2} \ldots\right]
\end{gathered}
$$

where $\boldsymbol{\Phi}_{p}$ and $\boldsymbol{\Phi}_{s}$ are mass normalized mode shapes of the uncoupled primary and secondary systems respectively. We can write 


$$
\begin{gathered}
\boldsymbol{U}=[\boldsymbol{\Phi}] . \boldsymbol{X}(t) \\
\boldsymbol{X}^{T}=\left[\begin{array}{ll}
\boldsymbol{X}_{\boldsymbol{P}}^{T} & \boldsymbol{X}_{S}^{T}
\end{array}\right] \\
\boldsymbol{X}_{\boldsymbol{P}}^{T}=\left[x_{p 1} x_{p 2} \ldots\right] ; \boldsymbol{X}_{\boldsymbol{s}}^{T}=\left[\begin{array}{lll}
x_{s 1} x_{s 2} \ldots
\end{array}\right]
\end{gathered}
$$

Pre-multiplying Equation (1.1) by $[\boldsymbol{\Phi}]^{T}$ and using Equation (1.3), the Equation (1.1) transforms into

$$
\{\ddot{\boldsymbol{X}}\}+[\overline{\boldsymbol{C}}]\{\dot{\boldsymbol{X}}\}+[\overline{\boldsymbol{K}}]\{\boldsymbol{X}\}=-\{\boldsymbol{\Gamma}\} . \ddot{u}_{g}
$$

$\{\boldsymbol{\Gamma}\}$ is the vector of modal participation vectors for the uncoupled systems defined as

$$
\begin{gathered}
\boldsymbol{\Gamma}^{T}=\left[\begin{array}{ll}
\boldsymbol{\Gamma}_{\mathbf{p}}{ }^{T} & \boldsymbol{\Gamma}_{\mathbf{s}}{ }^{T}
\end{array}\right] \\
\boldsymbol{\Gamma}_{\mathbf{p}}=\left[\begin{array}{lll}
\gamma_{\mathrm{p} 1} & \gamma_{\mathrm{p} 2} & \ldots
\end{array}\right], \quad \boldsymbol{\Gamma}_{\mathbf{s}}=\left[\begin{array}{lll}
\gamma_{\mathrm{s} 1} & \gamma_{\mathrm{s} 2} & \ldots
\end{array}\right]
\end{gathered}
$$

where $\gamma_{\mathrm{p} i}$ and $\gamma_{\mathrm{si}}$ are the modal participation factors of the primary and secondary systems respectively.

The elements of $[\overline{\boldsymbol{C}}]$ and $[\overline{\boldsymbol{K}}]$ matrices can be written in terms of the primary and secondary system frequencies, damping ratios, participation factors, the modal mass ratios $r_{i \alpha}$ and the increment in frequency due to static constraint imposed by the secondary system on the primary system, $\Delta \omega_{p i j}$ (Gupta, 1990). The elements of $[\overline{\boldsymbol{C}}]$ and $[\overline{\boldsymbol{K}}]$ matrices are given in Equations (1.6) - (1.7): 


$$
\begin{aligned}
\bar{K}_{i j} & =\omega_{p i}^{2}+\boldsymbol{\phi}_{c i}{ }^{T} \boldsymbol{K}_{c c}^{s} \boldsymbol{\phi}_{c i}, \quad i=j \\
& =\boldsymbol{\phi}_{c i}{ }^{T} \boldsymbol{K}_{c c}^{s} \boldsymbol{\phi}_{c j}=\Delta \omega_{p i j}^{2}+\sum_{\alpha} \omega_{s \alpha}^{2} \sqrt{r_{i \alpha} \cdot r_{j \alpha}}, \quad i \neq j \\
\bar{C}_{i j} & =2 \omega_{p i} \xi_{p i}+\boldsymbol{\phi}_{c i}{ }^{T} \boldsymbol{C}_{c c}^{s} \boldsymbol{\phi}_{c i}, \quad i=j \\
& =\boldsymbol{\phi}_{c i}{ }^{T} \boldsymbol{C}_{c c}^{s} \boldsymbol{\phi}_{c j}=\sum_{\alpha} 2 \omega_{s \alpha} \xi_{s \alpha} \sqrt{r_{i \alpha} \cdot r_{j \alpha}}, \quad i \neq j \\
\bar{K}_{i \alpha} & =\boldsymbol{\phi}_{c i}{ }^{T} \boldsymbol{K}_{c s} \boldsymbol{\phi}_{s \alpha}=-\omega_{s \alpha}^{2} r_{i \alpha}^{1 / 2} \\
\bar{C}_{i \alpha} & =\boldsymbol{\phi}_{c i}{ }^{T} \boldsymbol{C}_{c s} \boldsymbol{\phi}_{s \alpha}=-2 \omega_{s \alpha} \xi_{s \alpha} r_{i \alpha}^{1 / 2} \\
\bar{K}_{\alpha \beta} & =\omega_{s \alpha}^{2}, \quad \alpha=\beta \\
& =0, \quad \alpha \neq \beta \\
\bar{C}_{\alpha \beta} & =2 \omega_{s \alpha} \xi_{s \alpha}, \quad \alpha=\beta \\
& =0, \quad \alpha \neq \beta
\end{aligned}
$$

where $\omega_{p i}$ and $\xi_{p i}$ are the circular frequency and the damping ratio, respectively, for the $i^{\text {th }}$ uncoupled mode of the primary system; $\omega_{s \alpha}$ and $\xi_{s \alpha}$ are the corresponding values for the $\alpha^{\text {th }}$ secondary system mode. In Equations (1.6) - (1.7), subscript c denotes the primary system DOF which are connected with the secondary system; and subscript s denotes the secondary DOF. The matrices $K_{c c}^{s}$ and $C_{c c}^{s}$ are the stiffness and damping contributions of the secondary systems. The modal mass ratio $r_{i \alpha}$ is given by

$$
r_{i \alpha}^{2}=\phi_{c i}^{T} \gamma_{c \alpha}^{T} \gamma_{c \alpha} \phi_{c i}
$$


In typical building piping systems, the modal ratios are significantly very small (to the order of $10^{-4}$ or less). Also, relatively light secondary systems such as piping do not impart any meaningful static constraint to the primary system i.e. $\Delta \omega_{p i j} \rightarrow 0$.

The free vibration solution of the Equation (1.4) yields complex eigenvalues $\lambda_{i}$, and eigenvectors $\boldsymbol{\psi}_{i}$, which in turn could be used to determine the coupled frequencies $\omega_{i}$, coupled damping ratios $\zeta_{i}$ and mode shapes. The $i^{\text {th }}$ eigenvalue, $\lambda_{i}$ is written as

$$
\lambda_{i}=-\zeta_{i} \omega_{i}+i \omega_{D i}, \quad \omega_{D i}=\omega_{i} \sqrt{1-\zeta_{i}^{2}}
$$

Each complex eigenvector $\lambda_{i}$ and its conjugate $\overline{\lambda_{l}}$ give 2 real modal vectors $\boldsymbol{\Psi}_{\boldsymbol{i}}^{\boldsymbol{d}}$ and $\boldsymbol{\Psi}_{\boldsymbol{i}}^{\boldsymbol{v}}$.

$$
\boldsymbol{\Psi}_{\boldsymbol{i}}^{\boldsymbol{d}}=-2 \operatorname{Re} \overline{\lambda_{l}} F_{i} \boldsymbol{\psi}_{i}, \quad \boldsymbol{\Psi}_{\boldsymbol{i}}^{\boldsymbol{v}}=-2 \operatorname{Re} F_{i} \boldsymbol{\psi}_{i}
$$

where $F_{i}$ are given by

$$
F_{i}=\frac{1}{a_{i}} \boldsymbol{\psi}_{i}^{T} \boldsymbol{M} \boldsymbol{U}_{\boldsymbol{b}}, \quad a_{i}=2 \lambda_{i} \boldsymbol{\psi}_{i}^{T} \boldsymbol{M} \boldsymbol{\psi}_{i}+\boldsymbol{\psi}_{i}{ }^{T} \boldsymbol{C} \boldsymbol{\psi}_{i}
$$

In the time history analysis, the recorded ground acceleration data $\ddot{u_{g}}(t)$ can be used to perform the time step integration of the following SDOF equation of motion corresponding to each coupled mode $i$.

$$
\ddot{x}_{\imath}+2 \zeta_{i} \omega_{i} \dot{x}_{\imath}+\omega_{i}{ }^{2} x_{i}=-\ddot{u}_{g}(t)
$$


Equation (1.12) yields $x_{i}(t), \dot{x}_{l}(t)$ and $\ddot{x}_{l}(t)$ for each coupled mode $i$, after which the displacement vector $\boldsymbol{U}(t)$ is obtained by

$$
\begin{gathered}
\boldsymbol{U}(t)=\sum_{i=1}^{N} \boldsymbol{U}_{\boldsymbol{i}}(t), \quad \boldsymbol{U}_{\boldsymbol{i}}=\boldsymbol{U}_{\boldsymbol{i}}^{\boldsymbol{d}}(t)-\boldsymbol{U}_{\boldsymbol{i}}^{\boldsymbol{v}}(t) \\
\boldsymbol{U}_{\boldsymbol{i}}^{\boldsymbol{d}}(t)=\boldsymbol{\Psi}_{\boldsymbol{i}}^{\boldsymbol{d}} x_{i}(t), \quad \boldsymbol{U}_{\boldsymbol{i}}^{\boldsymbol{v}}(t)=\boldsymbol{\Psi}_{\boldsymbol{i}}^{\boldsymbol{v}} \dot{x}_{l}(t)
\end{gathered}
$$

In the Response Spectrum method, the spectral displacement $S_{D i}^{d}$ and spectral velocity $S_{V i}^{v}$ corresponding to coupled frequency and damping ratio of the $i^{\text {th }}$ coupled mode are defined as

$$
S_{D i}^{d}=\max \left|x_{i}(t)\right|, \quad S_{V i}^{v}=\max \left|\dot{x}_{l}(t)\right|
$$

The $S_{D i}^{d}$ and $S_{V i}^{v}$ are obtained from the input response spectrum. Equations (1.9) and (1.10) give

$$
\boldsymbol{U}_{i \max }^{d}=\boldsymbol{\Psi}_{i}^{d} \cdot S_{D i}^{d} ; \boldsymbol{U}_{i \max }^{v}=\boldsymbol{\Psi}_{i}^{v} \cdot S_{V i}^{v}
$$

For each complex mode $i$, Equation (1.10) gives two displacement vectors $\boldsymbol{U}_{i \max }^{d}$ and $\boldsymbol{U}_{i{ }_{\text {max }}}^{v}$. These vectors can be used to evaluate any response values, $\boldsymbol{R}_{i}^{d}$ and $\boldsymbol{R}_{i}^{v}$ for each mode. The combined responses are obtained by combining $\boldsymbol{R}_{i}^{d}$ and $\boldsymbol{R}_{i}^{v}$ in accordance with the rules for combining modal responses (Gupta et al, 1996). 


\subsection{Incorporation of Uncertainties in Primary-Secondary System Analysis}

The response of the secondary system is sensitive to uncertainties in both structural characteristics as well as loading. Uncertainty in excitation is commonly modeled as a random process by many researchers. Classical random vibration theory can be used to evaluate the response variability due to uncertain excitation (Lin, 1967). The effect of uncertainty in structural properties on the secondary system response has been studied by many researchers. Igusa and Der Kiureghian (1988) studied the reliability of uncertain systems subjected to stochastic excitation by modeling the frequencies and damping ratios as continuous random variables and employed first and second order reliability methods to evaluate the reliability of simple secondary systems. Sensitivity measures of the reliabilities with respect to the uncertain variables are derived as well. Jensen and Iwan (1992) developed a method for the dynamic analysis of linear systems with uncertain parameters to stochastic excitation and applied it to primary-secondary system to illustrate that uncertainty in stiffnesses can have a strong influence on the secondary system response. Huang et al (1994) studied the extent of response amplification and reliability reduction of secondary systems due to primary-structure yielding and tuning of the secondary system frequencies with the fundamental frequency of the primary system. Key (1999) examined the frequency dependence of the relationship between damping and response in single-degree-of-freedom systems and extended it to secondary systems. While the observations made in all the above 
studies are very similar, they cannot be directly used to account for uncertainties in coupled analysis by response spectrum method.

In the conventional analysis, several methods focus on modification of the auto-floor spectra (Liu et al, 1973; Singh, 1980; Igusa and Der Kiureghian, 1985; Chen, 1993; Reed et

al, 1994 etc.) The most widely employed techniques for accounting these uncertainties in conventional analysis are simplified methods referred to as "Peak-Broadening" and "Peakshifting" methods (USNRC, 1978).

\subsubsection{Peak Broadening \& Peak Shifting}

In Peak Broadening (ANSI/ASME BPV-III-I-A 2007, NUREG 1.122 1978), the narrow banded floor response spectra computed by analyzing the primary system, are smoothened and the peaks associated with the structural frequencies broadened. It is recommended that the amount of peak broadening associated with each primary structure frequency $f_{j}$, be determined from the variation in the structural frequency. This variation may be caused by uncertainties in the material properties of the structure and soil. The total variation in the primary structure frequency $\pm \Delta f_{j}$ is then computed by considering the square-root-of-sumof-squares of $0.05 f_{j}$ and the individual variations $\left( \pm \Delta f_{j}\right)_{n}$ caused due to each influencing parameter $n$. A minimum value of $0.1 f_{j}$ is recommended if the actual computed value of $\Delta f_{j}$ is less than $0.1 f_{j}$. If the above procedure is not used, then USNRC (1978) recommends that $\Delta f_{j}$ be taken as $0.15 f_{j}$. 
Peak shifting (ANSI/ASME BPV-III-I-A 2007) is recommended as an alternative to the peak broadening. This method is based on the premise that the actual natural frequency of the primary structure can assume only a single value within the frequency range defined by $f_{j} \pm \Delta f_{j}$. Consequently, for a given value of the primary system frequency, only one of the secondary system modes can receive the peak spectral acceleration associated with the given value. In this method, the secondary system frequencies $\left(f_{e}\right)_{N}$ which lie within the $\pm 15 \%$ range of variation in the primary system frequency $f_{j}$ are selected. If $N$ such secondary system frequencies exist, the secondary system responses are then evaluated by performing $N+3$ separate analysis. These $N+3$ analysis are performed by shifting the unbroadened floor spectrum by factors of $0,-0.15 f_{j},+0.15 f_{j}$ and $\frac{\left(\left(f_{e}\right)_{n}-f_{j}\right)}{f_{j}}$, where $n=1$ to $N$. This procedure is applied separately to floor spectra in three orthogonal directions if needed.

Both methods tend to increase conservatism in conventionally evaluated responses (Aradhya and Gupta, 1999). Moreover, such methods cannot be applied directly in a coupled analysis where the floor spectra are neither generated nor required.

Gupta and Choi (2005) modeled uncertainties in dynamic properties (natural frequencies and damping ratios of primary-secondary system) and ground motion input within a coupled system analysis framework using response spectrum method and evaluated design response of the secondary system statistically. In this study, a limited number of simulations were conducted for coupled systems with uncertain uncoupled frequencies and 
damping ratios using an input design spectrum. A method "Square-Root-of-mean-ofSquares" (SRMS) based on total probability theorem was proposed to obtain the design response $R_{0.84}$ as

$$
R_{0.84} \cong\left[\frac{1}{N} \sum_{i=1}^{N} R_{0.84 / Z_{i}}{ }^{2}\right]^{1 / 2}
$$

where $R_{0.84 / Z_{i}}$ is the conditional response for a given simulated set of modal parameters $Z_{i} \equiv\left(\omega_{i}, \xi_{i}\right)$ and $N$ is the total number of simulated sets of modal parameters. The SRMS method is described in detail in Chapter 4 of this thesis. The results from the SRMS method agreed well with the time history results for simple SDOF primary-SDOF secondary systems. However, the application of the SRMS procedure was not illustrated for multi degree of freedom (MDOF) primary - MDOF secondary systems. This thesis focuses on additional considerations required for implementation of SRMS method to MDOF primary - MDOF secondary systems.

\subsection{Objective}

The objective of this study is to investigate the effect of uncertainties in modal properties of MDOF primary - MDOF secondary systems and ground motion on secondary system response. The thesis focuses on verification and application of the SRMS approach (Gupta and Choi, 2005) to MDOF primary - MDOF secondary systems. Specific tasks needed to achieve the objective of the study are: 
- Task-1: Study the nature of variations in natural frequencies of an uncoupled MDOF system due to uncertainty in structural properties. This task focuses on understanding the nature of the probability density functions of natural frequencies of a structure and to evaluate if the probability density functions of the higher order modes have significant overlap.

- Task-2: Study the validity of the SRMS approach to the cases in which multiple modes of primary system may be tuned or nearly tuned to a secondary system mode. Such a case involves simulating random sets of closely-spaced natural frequencies.

B Sub-Task 2.1: Identify the limitations in application of the SRMS approach proposed by Gupta and Choi (2005) to this case. The purpose of this task is to evaluate if the independently simulated sets of natural frequencies in MDOF systems is correct. In cases where the probability density functions of modes with closely-spaced frequencies have significant overlap, it is anticipated that the independently sampled sets of natural frequencies may not be in correct order.

- Task-3: Use fundamental probability approaches to develop a procedure for simulation of frequencies in correct order. 
- Task-4: Interface the proposed approach with the SRMS method.

- Task-5: Use numerical examples to test the validity of the SRMS method for coupled systems with

B Varying degrees of tuning between the multiple modes of uncoupled primary and secondary systems.

B Multiply connected secondary systems with tuning between single and multiple modes of uncoupled systems.

\subsection{Organization}

Chapter 2 describes Task-1 listed above in Section 1.4 of this thesis. It also discusses the existing studies on eigenproperties' statistics. It is illustrated that when the individual probability density functions of modes with closely-spaced frequencies overlap significantly, independent sampling of the frequencies can result in incorrect order of frequencies in a MDOF system. This chapter establishes the need for developing a joint density function for closely-spaced frequencies (Task-2).

Chapter 3 describes formulation of a joint density function for modes with closelyspaced frequencies. This chapter addresses the Task-3 of the objectives listed in section 1.4 
of this thesis. The joint probability density function developed ensures that sampled natural frequencies always remain as an ordered set.

Chapter 4 presents a detailed overview of the SRMS method as developed by Gupta and Choi (2005).

Chapter 5 considers various numerical examples for verification of the SRMS method using MDOF primary - MDOF secondary systems as described in Task-5 of Section 1.4 of this thesis. The design response values obtained from SRMS method are compared with the design response value obtained from time history analyses.

Lastly, Chapter 6 discusses the conclusions as well as the limitations of this work and provides recommendations for future work. 


\section{CHAPTER - 2}

\section{MODELING UNCERTAINTY IN MODAL PROPERTIES FOR MDOF SYSTEMS}

Implementing the SRMS method comprises of conducting multiple analyses of the coupled system. This involves generation of multiple sets of random natural frequencies for uncoupled primary and secondary systems. In this chapter, we study the various considerations that need to be addressed in generation of these random sets. Let us begin by considering the natural frequencies of a structure obtained from the solution of an eigenvalue problem:

$$
\boldsymbol{K} \phi=\omega^{2} \boldsymbol{M} \phi
$$

where $\boldsymbol{K}$ and $\boldsymbol{M}$ are the stiffness and mass matrices of the structure. The statistics of the frequencies in turn depend on the statistics of the elements of mass and stiffness matrices. Any uncertainty in these matrices induces randomness in all natural frequencies to some extent. Consequently, the frequencies tend to be correlated with each other to some degree. Furthermore, the probability density functions of closely-spaced frequencies may overlap significantly. Overlap of such probability density functions can give incorrect set of frequencies when sampled independently in the sense that the modal frequencies do not remain as ordered pairs or ordered set. The frequencies of any uncoupled primary or 
secondary system should all be in ascending order. So, considering correlations between closely-spaced frequencies in some way is necessary for correct sampling.

In order to evaluate these two aspects, we begin by examining the case of a simply supported beam of length $L$, modulus of elasticity $E$, moment of inertia $I$ and mass per unit length $m$. The $n^{\text {th }}$ order frequency, $\omega_{n}$ of the beam (Humar, 2002) is given by

$$
\omega_{n}=n^{2} \pi^{2} \sqrt{\frac{E I}{m L^{4}}}
$$

Equation (2.2) can be rewritten to describe the relationship between the $n^{\text {th }}$ order frequency, $\omega_{n}$ and flexural rigidity $R$ as

$$
\omega_{n}=D_{n} \sqrt{R} \quad \text { where } D_{n}=\frac{n^{2} \pi^{2}}{\sqrt{m L^{4}}} \text { and } R=E I
$$

For any two random variables $X$ and $Y$ with probability density functions $f_{X}(x)$ and $f_{Y}(y)$ respectively and given that $y=g(x)$, we have

$$
f_{Y}(y)=f_{X}\left(g^{-1}(y)\right) \cdot\left|\frac{d X}{d Y}\right|
$$

From Equation (2.3),

$$
\frac{d R}{d \omega_{n}}=\frac{2 \omega_{n}}{D_{n}^{2}}
$$


If $\Omega_{n}$ and $R$ be the random variables representing the $n^{\text {th }}$ frequency $\omega_{n}$ of the beam and flexural rigidity $E I$ respectively, the Equations (2.3) - (2.5) yield

$$
f_{\Omega_{n}}\left(\omega_{n}\right)=\frac{2 \omega_{n}}{D_{n}^{2}} f_{R}\left(r=\frac{\omega_{n}^{2}}{D_{n}^{2}}\right)
$$

where $f_{\Omega_{n}}\left(\omega_{n}\right)$ is the probability density function of $n^{\text {th }}$ order natural frequency of the beam and $f_{R}(r)$ is the probability density function of variations in flexural rigidity of the beam.

Figure 2.1 plots the probability density functions of the first 10 natural frequencies as given by Equation (2.6) of a 12 meter long simply supported concrete beam with a rectangular cross-section of height $1.1 \mathrm{~m}$ and width $0.25 \mathrm{~m}$. The Young's modulus and density of concrete are taken as $25 \mathrm{GPa}$ and $2400 \mathrm{Kg} \cdot \mathrm{m}^{-3}$ respectively. The rigidity $R$ is assumed to be normally distributed with a coefficient of variation of 0.15 . It can be seen that the density functions of the frequencies for lower order modes are relatively narrow banded and do not overlap in any significant way. However, there is a significant degree of overlap among the density functions for the frequencies of higher modes.

From Equation (2.2), the $i^{\text {th }}$ and $j^{\text {th }}$ frequency are related as

$$
\omega_{j}=\left(\frac{j^{2}}{i^{2}}\right) \omega_{i}
$$

Equation (2.7) indicates a linear relationship between $\omega_{i}$ and $\omega_{j}$. This implies that the correlation coefficient $\rho_{\Omega_{i}, \Omega_{j}}=1.0$. 

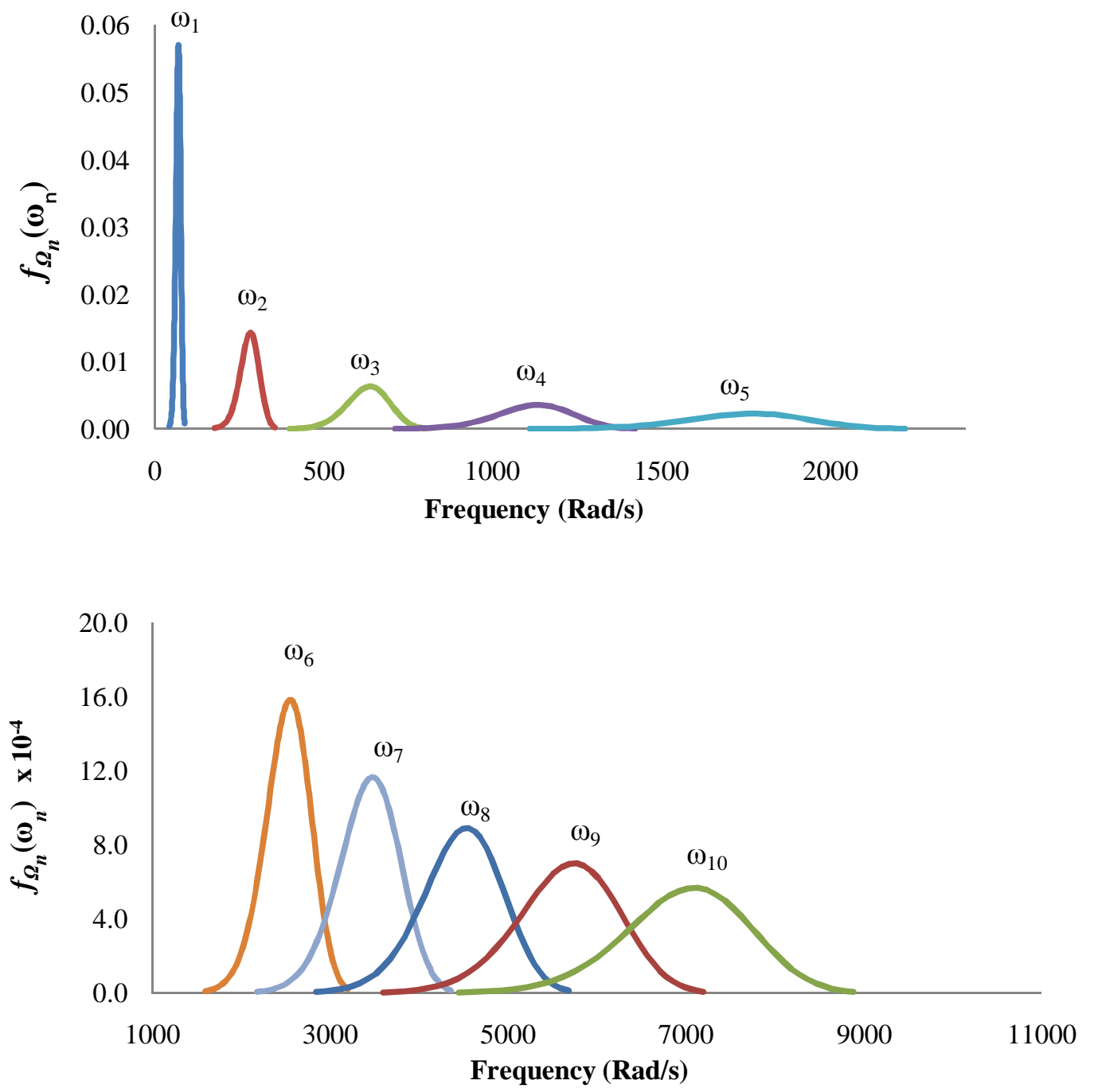

Figure 2.1: Probability density function of first 10 natural frequencies of a beam 
In the above example, a closed-form expression for probability density functions of the natural frequencies was derived based on a variation in a single parameter i.e. flexural rigidity $(E I)$. Similar expressions can be derived for a shear beam considering variations in shear rigidity parameter (see Appendix - A). Though, the probability density functions of natural frequencies can be seen to be over-lapping, the natural frequencies can be independently simulated without any problem because the correlation between any two frequencies is unity. However, the correlation between the frequencies will not always necessarily be unity as illustrated by the following example.

Consider a discretized 4-storey structure modeled as shown in Figure 2.2. $\mathrm{K}_{i}$ and $\mathrm{M}_{i}$ represent the storey stiffness and mass associated with $i^{\text {th }}$ storey respectively.

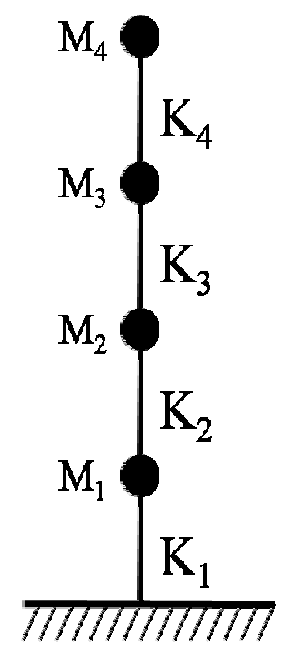

For $i=1,2$

$\mathrm{M}_{i}=100 \mathrm{Kg} ; \mathrm{K}_{i}=100000 \mathrm{~N} \cdot \mathrm{m}^{-1}$

For $i=3,4$

$\mathrm{M}_{i}=25 \mathrm{Kg} ; \mathrm{K}_{i}=25000 \mathrm{~N} \cdot \mathrm{m}^{-1}$

Figure 2.2: A 4-DOF system considered to evaluate the correlations between the natural frequencies 
The natural frequencies of the structure are 2.3, 4.1, 7.9 and $8.6 \mathrm{~Hz}$ respectively. All $\mathrm{K}_{i}$ are modeled as independent random variables varying uniformly within $\pm 15 \%$ of their respective means. A Monte Carlo simulation with 5000 sample sets of random stiffnesses is conducted to evaluate the natural frequencies of the system each time, and the correlation matrix $\rho$ is presented in Equation (2.8).

$$
\rho=\left[\begin{array}{cccc}
1.0 & 0.7857 & 0.6756 & 0.7288 \\
0.7857 & 1.0 & 0.6091 & 0.6967 \\
0.6756 & 0.6091 & 1.0 & 0.8071 \\
0.7288 & 0.6967 & 0.8071 & 1.0
\end{array}\right]
$$

where $\rho_{i j}$ is the correlation coefficient between $i^{\text {th }}$ and $j^{\text {th }}$ natural frequencies of the system.

It can be seen that if an uncertainty exists in multiple structural parameters, the correlation between the frequencies are not always unity though they are strongly correlated. However, obtaining the correlation coefficients between frequencies in this manner for a large scale structure is highly impractical. The next section presents a summary of existing studies in evaluation of statistics of natural frequencies of a structure.

\subsection{Existing Studies in Evaluation of Eigenproperties' Statistics}

Early work in obtaining the statistics of natural frequencies with variations in structural parameters employed perturbation methods. In a typical perturbation method, the mass and stiffness matrices are considered to be non-linear functions of some random vector $\mathbf{x} . \mathbf{x} \in \mathbb{R}^{m}$ 
can denote all the physical, material and geometrical parameters of the structure. The mass and stiffness matrices are represented as $\boldsymbol{M}(\mathbf{x})$ and $\boldsymbol{K}(\mathbf{x})$.

If the mean of $\mathbf{x}$ is $\boldsymbol{\mu} \in \mathbb{R}^{m}$ and considering

$$
\boldsymbol{M}(\boldsymbol{\mu})=\overline{\boldsymbol{M}} \text { and } \boldsymbol{K}(\boldsymbol{\mu})=\overline{\boldsymbol{K}}
$$

where $\overline{\boldsymbol{M}}$ and $\overline{\boldsymbol{K}}$ are the 'deterministic parts' of the mass and stiffness matrices of the structure. The deterministic part of the eigenvalues $\bar{\omega}_{i}=\omega_{i}(\boldsymbol{\mu})$ is obtained from solution of the eigenvalue problem:

$$
\overline{\boldsymbol{K}} \bar{\phi}_{\iota}=\bar{\omega}_{i}^{2} \overline{\boldsymbol{M}} \bar{\phi}_{\imath}
$$

It is assumed that $\omega_{i}(\mathbf{x})$ is a continuous, differentiable function of the random parameter vector $\mathbf{x}$. In the mean-centered perturbation approach, $\omega_{i}(\mathbf{x})$ is expanded by its Taylor series about the point $\mathbf{x}=\boldsymbol{\mu}$ :

$$
\begin{aligned}
\omega_{i}(\mathbf{x})=\bar{\omega}_{i}+ & \mathbf{d}_{\omega_{i}}^{\mathrm{T}}(\boldsymbol{\mu})(\mathbf{x}-\boldsymbol{\mu})+\frac{1}{2 !}(\mathbf{x}-\boldsymbol{\mu})^{\mathrm{T}} \mathbf{D}_{\omega_{i}}(\boldsymbol{\mu})(\mathbf{x}-\boldsymbol{\mu}) \\
& +\cdots
\end{aligned}
$$

where

$$
\left\{\mathbf{d}_{\omega_{i}}(\boldsymbol{\mu})\right\}_{k}=\left.\frac{\partial \omega_{i}(\mathbf{x})}{\partial x_{k}}\right|_{\mathbf{x}=\boldsymbol{\mu}}
$$




$$
\left\{\mathbf{D}_{\omega_{i}}(\boldsymbol{\mu})\right\}_{k l}=\left.\frac{\partial^{2} \omega_{i}(\mathbf{x})}{\partial x_{k} \partial x_{l}}\right|_{\mathbf{x}=\boldsymbol{\mu}}
$$

The matrices $\mathbf{d}_{\omega_{i}}(\boldsymbol{\mu}) \in \mathbb{R}^{m}$ and $\mathbf{D}_{\omega_{i}}(\boldsymbol{\mu}) \in \mathbb{R}^{m \times m}$ represent the sensitivities of the natural frequency $\omega_{i}(\mathbf{x})$ with respect to changes (or "perturbations") in elements of $\mathbf{x}$. The series expansion can be truncated after one (first-order perturbation) or two terms (secondorder perturbation) by neglecting the higher order terms for small perturbations. One may refer to (Kozin and Klosner, 1989; Lin and Cai, 2004) for a detailed discussion on perturbation methods.

Many earlier studies employed this methodology to obtain the statistics of the natural frequencies of a structure. Soong and Bogdanoff (1963) studied discretized shear beams with random stiffness and masses and obtained individual probability density functions of natural frequencies. Collins and Thomson (1969) employed the first order perturbation expansion (linear) to derive the second moment statistics of the frequencies and mode shapes of MDOF systems establishing that if the random variables in $\mathbf{x}$ are assumed to be Gaussian, the resulting natural frequencies results in a joint Gaussian distribution of natural frequencies. Similar approach had been employed by Shinozuka and Astill (1972); Hasselman and Hart (1972); Hart (1973); Song et al (1995). However, the mean-centered perturbation approaches yield agreeable results only when the perturbations are assumed to be small.

Adhikari and Friswell (2007) addressed this "small-randomness" assumption associated with mean-centered methods and proposed a new method based on perturbation 
expansion about an optimal point other than the mean. The same paper along with Adhikari (2007) illustrates a method of deriving joint probability density function of natural frequencies based on asymptotic approximation of multidimensional integrals to obtain a closed-form solution of a generalized $r^{\text {th }}$ moment of the eigenvalues. These methods are able to predict the higher moment statistics of natural frequencies satisfactorily but the methods do not give accurate results for structures with closely-spaced frequencies.

Given the practical difficulties of characterizing the probabilistic information (density functions, correlations between different variables etc.) for uncertain structural parameters linked with the above cited studies, some researchers (Gao, 2006; Modares et al, 2006; Sim et al, 2007) even employed 'possibilistic' approaches to treatment of uncertainty where the properties are assumed to lie within certain ranges without specifying any probability distributions, and the bounds of natural frequencies are computed by interval calculus methods. Estimation of such bounds for each physical parameter in a large-scale structural model is impractical. Consequently, some researchers have developed non-parametric methods of determining joint distributions.

The Principle of Maximum Entropy has been used in wide areas of structural engineering research to determine probability density functions when only minimal data (average values of some moments of the variables) is available. The entropy $H$ of a probability distribution function is the measure of uncertainty associated with the random variable. For a one-dimensional probability density function $f_{X}(x)$, the entropy is defined as 


$$
H=-\int_{-\infty}^{+\infty} f_{X}(x) \cdot \ln \left[f_{X}(x)\right] d x
$$

Given a "state-of-knowledge" about the distribution, it is possible to find a probability distribution function whose entropy is a maximum for the given information. Such a distribution is generally regarded as the most unbiased approximate density function befitting the "state-of-knowledge".

Sobczyk and Trebicki (1990) demonstrated that the principle of maximum entropy is a formidable tool for solving stochastic differential equations and derived the probability distribution of the response of a harmonic oscillator subjected to random non-Gaussian excitation. Soize (2001) presented an approach of modeling random uncertainties of a mechanical system by a nonparametric model using entropy optimization principle to predict transient responses subjected to impulse loading in the context of linear structural dynamics. Livingston and Shuang (2005) used maximum entropy method to estimate the probability density function of nonlinear or chaotic behavior in structural health monitoring data. Adhikari and Friswell (2006) used the Maximum Entropy Method to obtain the joint distribution of natural frequencies having determined the moments of eigenvalues. Mignolet and Soize (2008) formulated a non-parametric probabilistic model based on maximization of entropy under a set of constraints representing standard deviations of eigenvalues. While the principle of maximum entropy can be used to determine the joint distribution of natural frequencies given the information about their moments, the procedure is computationally 
intensive for even simple systems and can be quite impractical to implement in real life complex structures.

All the above studies concentrate on the variability of natural frequencies considering uncertainty in structural parameters. Another factor that can cause a large variation in natural frequencies of the primary system is the soil-structure interaction (SSI). Hamilton and Hadjian (1976) studied the variation statistics of natural frequencies due to variations in material properties for fixed-base structures and as well as structures with soil-structure interaction. A study on real-time structural monitoring of a building by Clinton et al (2006) established that factors like moderate local seismic activity, saturation of the soil, extreme temperature conditions and winds can significantly, over time, shift the natural frequencies of buildings.

It is a complex exercise to consider all possible uncertainties in physical and geometrical parameters of a full scale structural model to obtain a joint distribution function or correlations between the natural frequencies that can be used to sample the random sets. Also, knowing or estimating the correlations between the natural frequencies of a structure does not solve the sampling problem completely. To illustrate this, let us assume that we arrive at a correlation matrix as given by Equation (2.8) for the 4-storey structure in Figure 2.2 earlier in this chapter. Assuming all the four natural frequencies to be Gaussian with a coefficient of variation of $0.15,5000$ sets of random natural frequencies are generated using the correlation matrix. It is found that $17 \%$ of the samples are still not in order (while only 
$65.6 \%$ of the samples are in order if the frequencies are independently sampled). Thus, explicit consideration of correlation coefficients between the natural frequencies may not ensure correct sampling unless the frequencies are perfectly correlated which is not always the case. The subsequent discussion suggests a method for sampling natural frequencies of an uncoupled system that avoids considering unordered sets.

\subsection{Necessary Constraints for Sampling of Natural Frequencies}

USNRC (1978) recommends a $\pm 15 \%$ uncertainty in the frequencies of the primary system in order to account the effect of uncertainties on secondary system response. It is reasonable to consider a uniform distribution of these frequencies within the prescribed frequency region of interest. Furthermore, it may be observed that a uniform distribution is the maximum entropy distribution of a random variable in a bounded interval among all continuous distributions supported in that interval. The SRMS method proposed by Gupta and Choi (2005) samples the frequencies of the uncoupled systems by treating each frequency as an independent random variable. This sampling is acceptable when the frequencies are far apart. For two modes with closely spaced frequencies, if the higher limit of the lower order mode's frequency range is greater than the lower limit of the higher order mode's frequency range,

there is a chance of that sampled frequencies do not remain in order. If $\mu_{1}$ represents the 
mean frequency of the lower order mode and $\mu_{2}$ represents the mean frequency of the subsequent higher order mode, this condition can be represented as

$$
1.15 \mu_{1}>0.85 \mu_{2}
$$

which implies $\mu_{2}<1.353 \mu_{1}$ i.e. if the mean frequencies are closer by $35.3 \%$ or less, the chance of incorrect sampling increases significantly with increasing closeness of the frequencies. Consequently, any sampling scheme should consider a definite constraint that $P\left(\Omega_{2}<\Omega_{1}\right)=0$ where $\Omega_{1}$ and $\Omega_{2}$ denote the random variables representing two closely-spaced frequencies respectively.

\subsubsection{Problem in Rejection of Disordered Samples}

A simple consideration of the above constraint in the simulation process does not give correct results. While a simulation can begin with the assumption that random variables $\Omega_{1} \& \Omega_{2}$ representing the frequencies of two modes are uniformly distributed between $\left(0.85 \mu_{1}\right.$, $\left.1.15 \mu_{1}\right)$ and $\left(0.85 \mu_{2}, 1.15 \mu_{2}\right)$ respectively, an explicit constraint $P\left(\Omega_{2}<\Omega_{1}\right)=0$ considering both frequencies to be independent gives the sample sets for both $\Omega_{1} \& \Omega_{2}$ that do not result in uniformly distributed density functions as illustrated by the following example.

Let us consider $\mu_{1}=10 \mathrm{rad} / \mathrm{s}$ and $\mu_{2}=11 \mathrm{rad} / \mathrm{s}$. A total of 5000 samples are simulated for $\Omega_{1}$ and $\Omega_{2}$ are by assuming uniform distribution within $\pm 15 \%$ of $\mu_{1}$ and $\mu_{2}$ respectively.

Since, $P\left(\Omega_{2}<\Omega_{1}\right)=0$, only those samples $\left(\omega_{1}, \omega_{2}\right)$ where $\omega_{2}>\omega_{1}$ are considered. 
These simulated values are used to plot the probability density functions for both $\Omega_{1}$ and $\Omega_{2}$ in Figure 2.3. As seen in this figure, the individual distributions are not uniform.

Therefore, the implementation of such a constraint would require a joint density function for $\Omega_{1}$ and $\Omega_{2}$. In the next chapter, a formulation of a joint probability density function $f_{\Omega_{1}, \Omega_{2}}\left(\omega_{1}, \omega_{2}\right)$ is derived such that the constraint $P\left(\omega_{2}<\omega_{1}\right)=0$ always holds good. 

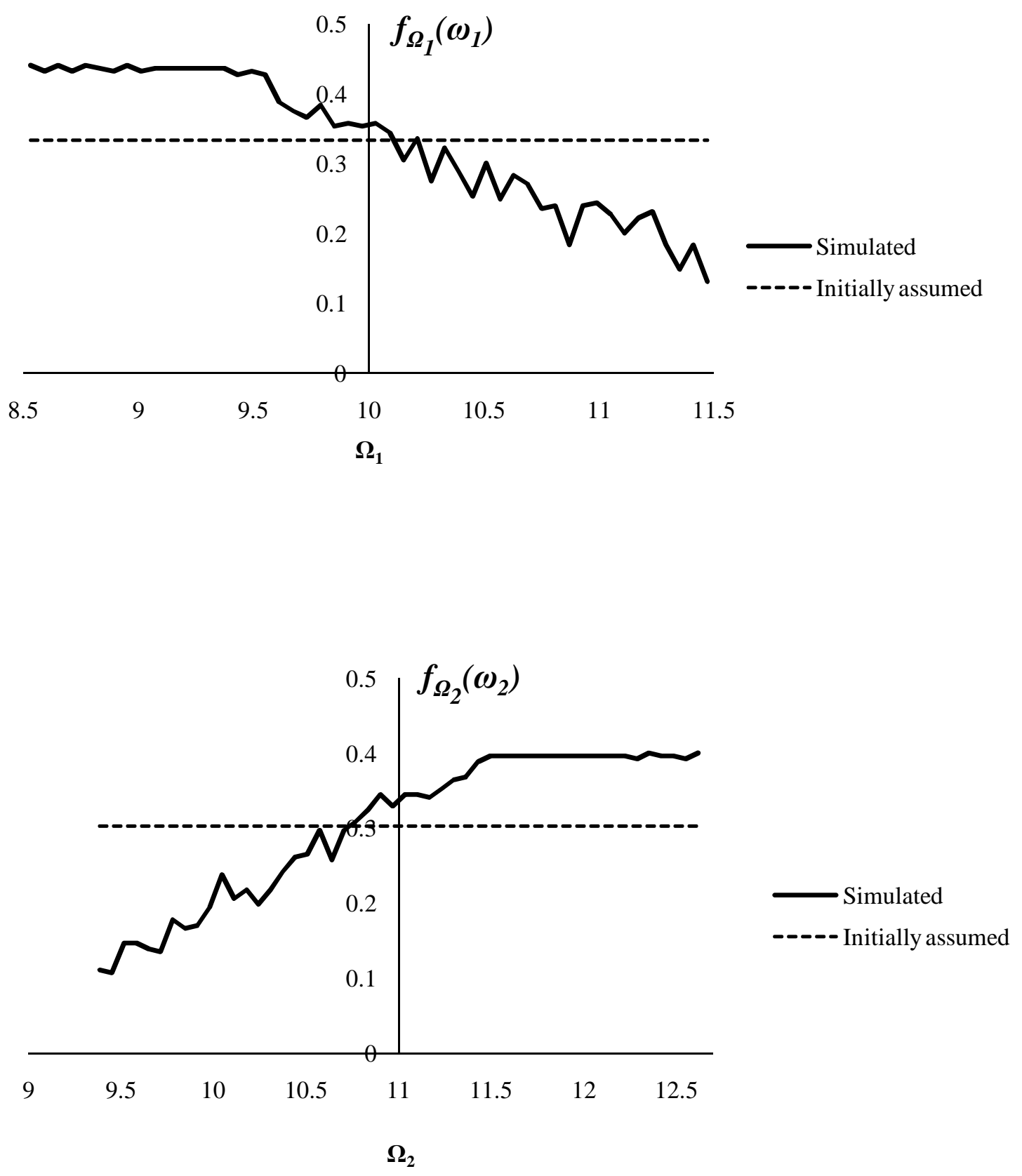

Figure 2.3: Probability Density Functions of $\Omega_{1}$ and $\Omega_{2}$ 


\section{CHAPTER - 3 \\ JOINT PROBABILITY DENSITY FUNCTION OF NATURAL \\ FREQUENCIES OF A STRUCTURE}

In the previous chapter, it was illustrated that considering the natural frequencies of a structure to be independent random variables can result in incorrect sampling when the frequencies are closely-spaced and their density functions overlap. Moreover, rejecting the incorrect samples result in individual density functions that are significantly different from the intended distribution for each natural frequency. Hence, this necessitates the need for a joint distribution that

- Produces the random samples of frequencies as an ordered set

- Maintains the individual density functions of all natural frequencies to be uniformly varying between $\pm 15 \%$ about their respective means.

In this chapter, we present a formulation for the joint probability density function that overcomes the problems encountered in the simulation of closely-spaced frequencies. Let $\left(\Omega_{1}, \Omega_{2} \ldots \Omega_{n}\right)$ represent a random sample set of natural frequencies of a structure. The formulation describes a joint probability density function between any two successive natural frequencies $\left(\Omega_{k-1}, \Omega_{k}\right)$ in a structure. 


\subsection{Problem Statement}

Let $\Omega_{1} \& \Omega_{2}$ represent the random variables for the two successive frequencies such that they are uniformly distributed within $\pm 15 \%$ of their respective mean values $\mu_{1}$ and $\mu_{2}$. A joint density function $f_{\Omega_{1}, \Omega_{2}}\left(\omega_{1}, \omega_{2}\right)$ is to be determined such that the density functions $f_{\Omega_{1}}\left(\omega_{1}\right)$ and $f_{\Omega_{2}}\left(\omega_{2}\right)$ obtained from the simulated values are uniform. The constraint $P\left(\omega_{2}<\right.$ $\left.\omega_{1}\right)=0$ can be rewritten as $f_{\Omega_{1}, \Omega_{2}}\left(\omega_{1}, \omega_{2}\right)=0$ when $\omega_{2}<\omega_{1}$. We can write

$$
\begin{aligned}
& \int_{0.85 \mu_{1}}^{1.15 \mu_{1}} f_{\Omega_{1}, \Omega_{2}}\left(\omega_{1}, \omega_{2}\right) d \omega_{1}=f_{\Omega_{2}}\left(\omega_{2}\right)=\frac{1}{B_{2}} \\
& \int_{0.85 \mu_{2}}^{1.15 \mu_{2}} f_{\Omega_{1}, \Omega_{2}}\left(\omega_{1}, \omega_{2}\right) d \omega_{2}=f_{\Omega_{1}}\left(\omega_{1}\right)=\frac{1}{B_{1}}
\end{aligned}
$$

where $B_{1}=1.15 \mu_{1}-0.85 \mu_{1}=0.3 \mu_{1}$ and $B_{2}=0.3 \mu_{2}$

Also,

$$
\mathrm{f}_{\Omega_{1}, \Omega_{2}}\left(\omega_{1}, \omega_{2}\right)=0 \forall \omega_{2}<\omega_{1}
$$

The individual distributions for $\Omega_{1} \& \Omega_{2}$ are shown in Figure 3.1 where the width of the overlap region $\bar{\omega}$ is defined as

$$
\bar{\omega}=1.15 \mu_{1}-0.85 \mu_{2}
$$




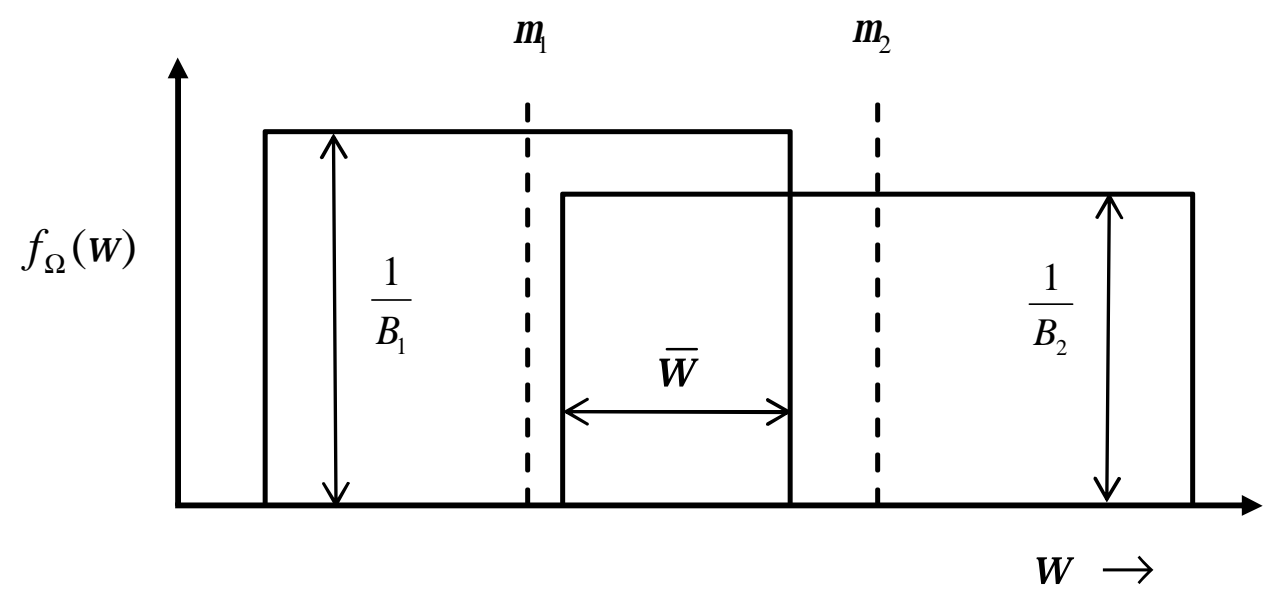

Figure 3.1: Individual probability density functions of $\omega_{1} \& \omega_{2}$

The possible sample space is represented in Figure 3.2. As we can see, any sampled point $\left(\omega_{1}, \omega_{2}\right)$ can lie only in the region $O D E B C$.

To determine the joint density function, it would be convenient to work with transformed random variables $X$ and $Y$ representing $\Omega_{1} \& \Omega_{2}$ respectively:

$$
\begin{aligned}
& X=\Omega_{1}-0.85 \mu_{1} \\
& Y=\Omega_{2}-0.85 \mu_{2}
\end{aligned}
$$

Note that $X$ is uniformly distributed between 0 and $B_{l}$ and $Y$ is uniformly distributed between 0 and $B_{2}$. Defining $\alpha$ and $\beta$ as 


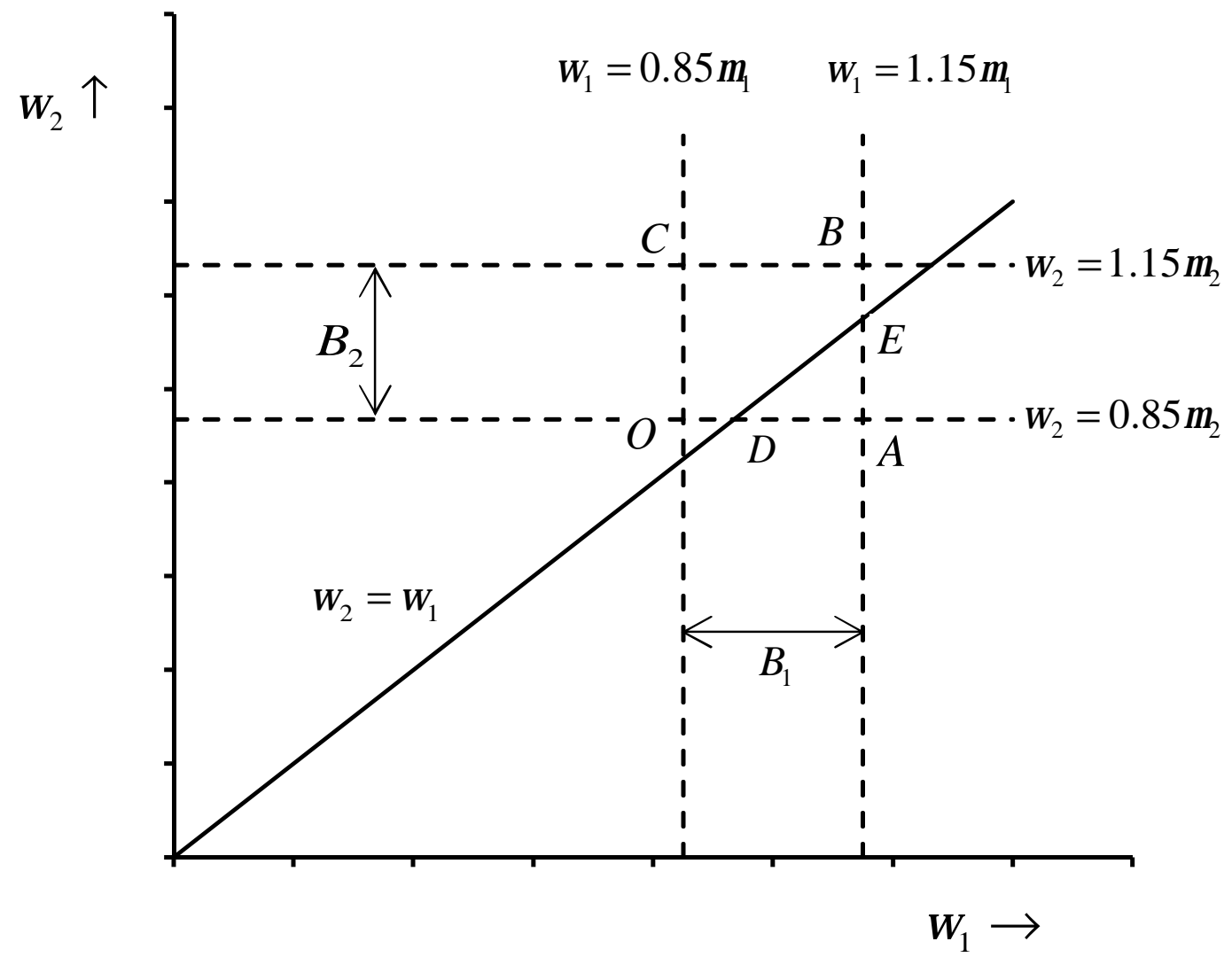

Figure 3.2: The sampling space

$$
\begin{aligned}
& \alpha=B_{1}-\bar{\omega} \\
& \beta=B_{2}-\bar{\omega}
\end{aligned}
$$

The problem space can be now represented in the transformed coordinates as shown in Figure 3.3. The physical dimensions for $\alpha$ and $\beta$ are shown in the figure. 


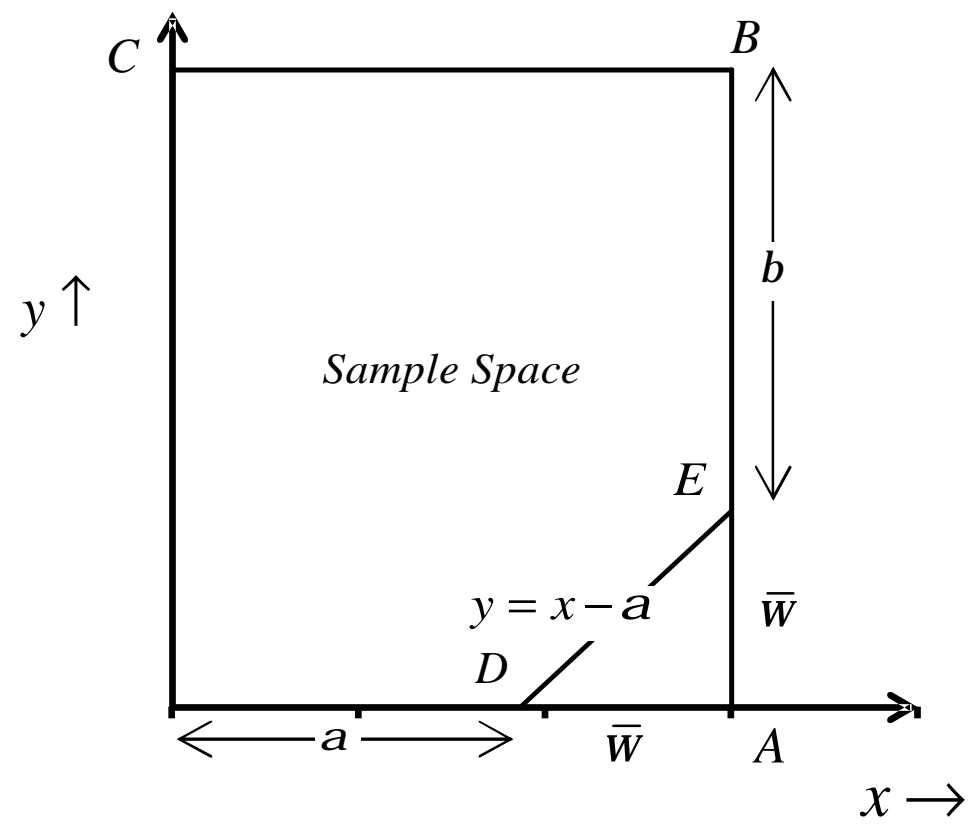

Figure 3.3: The transformed space

If the joint probability density function between $X$ and $Y$ is denoted by $f_{X, Y}(x, y)$, then we can write

$$
f_{\Omega_{1}, \Omega_{2}}\left(\omega_{1}, \omega_{2}\right)=f_{X, Y}\left(\omega_{1}-0.85 \mu_{1}, \omega_{2}-0.85 \mu_{2}\right)
$$

Notice that the line $\omega_{2}=\omega_{1}$ becomes $y=x-\alpha$ upon transformation. We now proceed to find $f_{X, Y}(x, y)$ such that

$$
\int_{0}^{B_{1}} f_{X, Y}(x, y) d x=f_{Y}(y)=\frac{1}{B_{2}}
$$




$$
\int_{0}^{B_{2}} f_{X, Y}(x, y) d y=f_{X}(x)=\frac{1}{B_{1}}
$$

Subject to the constraint that

$$
f_{X, Y}(x, y)=0 \forall y<x-\alpha
$$

\subsection{Central Symmetry of the Distribution}

In order to develop a formulation for $f_{X, Y}(x, y)$, it is important to understand the property of centrally symmetric distributions. The distribution of a random vector $\boldsymbol{X}$ is centrally symmetric (or "reflectively" or "diagonally" or "simply" or "antipodally" symmetric) about $\boldsymbol{\theta}$ if

$$
\boldsymbol{X}-\boldsymbol{\theta} \equiv \boldsymbol{\theta}-\boldsymbol{X}
$$

The density, if it exists, satisfies $f(\boldsymbol{\theta}-\boldsymbol{x})=f(\boldsymbol{x}-\boldsymbol{\theta})$, so that the Equation (3.13) represents the most direct nonparametric extension of uni-variate symmetry (Serfling, 1999).

Bi-variate uniform random variables are independent if and only is their joint probability density function is constant (Kimeldorf and Sampson, 1975). The joint density in this case cannot be a constant because of a constraint. On the other hand, the evaluation of joint density function would be simplified if the joint density function is assumed to be centrally-symmetric. In the case of two modes with closely spaced frequencies, the 
assumption of central symmetry appears reasonable because the frequencies tend to be strongly and positively correlated as observed in the previous chapter. The assumption, however, would not hold when the two frequencies are negatively correlated.

Assuming $f_{X, Y}(x, y)$ to be centrally symmetric, we can write

$$
f_{X, Y}(x, y)=f_{X, Y}\left(B_{1}-x, B_{2}-y\right)
$$

The location of points $(x, y)$ and $\left(B_{1}-x, B_{2}-y\right)$ in a centrally-symmetric sample space is illustrated in Figure 3.4.

Let us now consider the constraint $y=x-\alpha$ in the sample space of $f_{X, Y}(x, y)$. If the volume below $y=x-\alpha$ is non-existent then the volume above $y=x+\beta$ would also be zero. So, the effective sampling space is confined between the two parallel lines $D E$ and $F G$ as shown in Figure 3.5. The effective sampling space is denoted by the region $O D E B G F$ in Figure 3.5.

The constraints can be thus summarized as

$$
\begin{aligned}
& f_{X, Y}(x, y)=0 \text { if } y<x-\alpha \\
& f_{X, Y}(x, y)=0 \text { if } y>x+\beta
\end{aligned}
$$




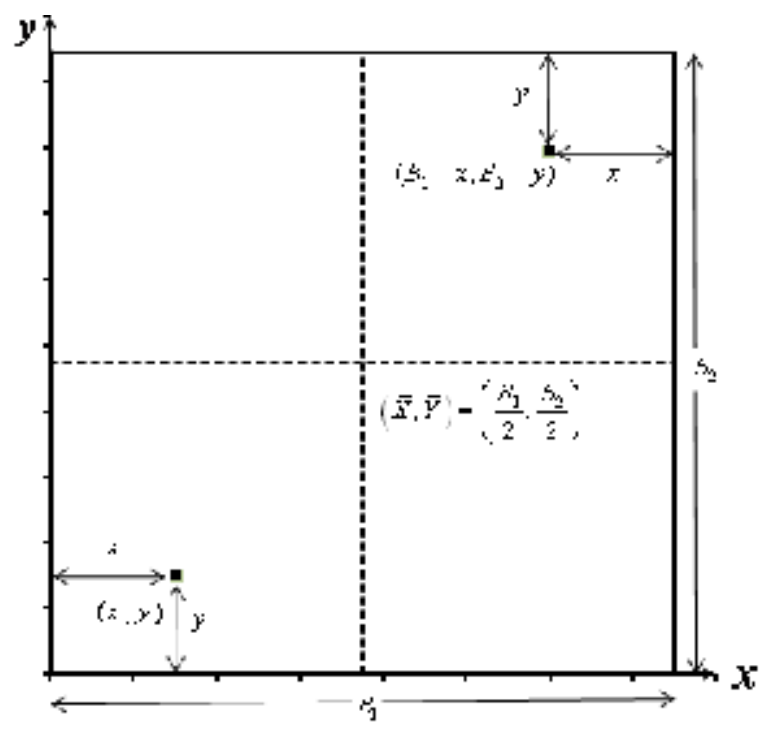

Figure 3.4: Illustration of Central symmetry in the joint density

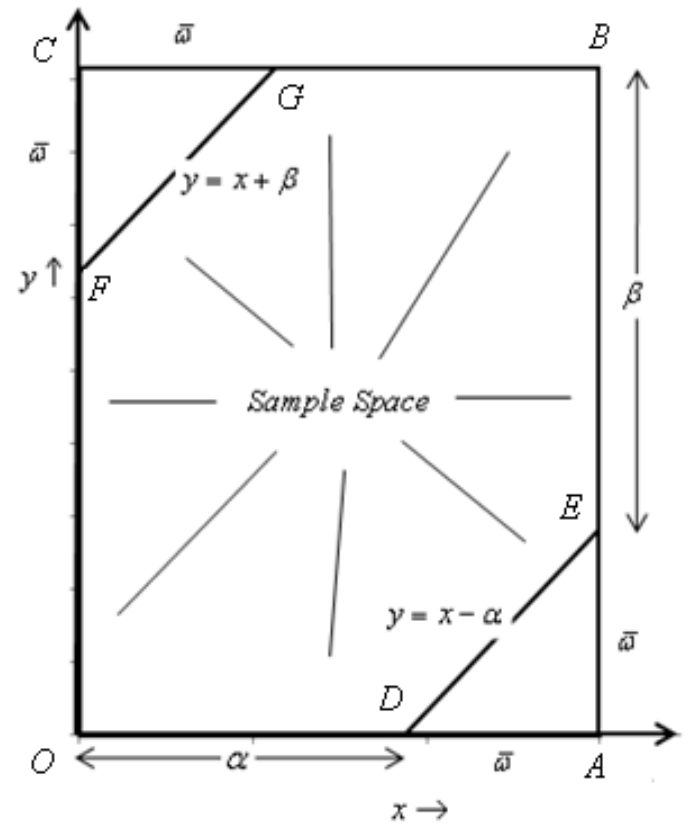

Figure 3.5: Effective sample space due to central symmetry of the density 


\subsection{Derivation of the Bi-variate Joint Probability Density Function}

Let us start by considering the simplest case in which $\Omega_{1}, \Omega_{2}$ (or $X, Y$ ) are simulated as independent variables. For independent variables with no constraint, the joint density function would simply be:

$$
f_{0}(x, y)=\frac{1}{B_{1}} \cdot \frac{1}{B_{2}}
$$

The total volume under this surface is 1.0 as should be the case for any probability density function. When the constraints $y=x-\alpha$ and $y=x+\beta$ are imposed, the volume under the surface of the sample space shown in Figure 3.5 will no longer remain unity. Since $f_{0}(x, y)$ is a constant surface, the volume lost due to the constraints can be given by the area bounded by the constraints multiplied by the value of $f_{0}(x, y)$. We can see from the Figure 3.5 that the area bounded by each of the constraints described in Equations (3.15) and (3.16) is $\frac{1}{2} \bar{\omega}^{2}$. Therefore, the volume lost by each constraint is $\frac{1}{2} \bar{\omega}^{2} f_{0}(x, y)$. From Equation (3.17), the total volume lost due to both the constraints is $\frac{\bar{\omega}^{2}}{B_{1} \cdot B_{2}}$. The volume under $f_{0}(x, y)$ is $\left(1-\frac{\bar{\omega}^{2}}{B_{1} \cdot B_{2}}\right)$.

Therefore, the definition for the joint density function $f_{X, Y}(x, y)$ would require addition of this lost volume. To do so, an additional layer $f_{1}(x, y)$ is superimposed onto $f_{0}(x, y)$ within the constraint space: 


$$
f_{X, Y}(x, y)=f_{0}(x, y)+f_{1}(x, y)
$$

Where,

$$
\int_{\substack{\text { Constrained } \\ \text { Space }}} f_{1}(x, y) d x d y=\frac{\bar{\omega}^{2}}{B_{1} \cdot B_{2}}
$$

Since $f_{X, Y}(x, y)$ is assumed to be centrally symmetric, $f_{1}(x, y)$ should be centrally symmetric as well.

In order to achieve uniform density for $\Omega_{1}$ and $\Omega_{2}$, the integrated volume as shown in Figure 3.6 would be

$$
\begin{aligned}
& \int_{0}^{y+\alpha} f(x, y) d x=\int_{0}^{B_{1}} f_{0}(x, y) d x=\frac{1}{B_{2}} \text { for any } y<\bar{\omega} \\
& \int_{0}^{x+\beta} f(x, y) d y=\int_{0}^{B_{2}} f_{0}(x, y) d y=\frac{1}{B_{1}} \text { for any } x<\bar{\omega}
\end{aligned}
$$

From Equation (3.19), we can rewrite Equation (3.21) as

$$
\begin{aligned}
& \int_{0}^{y+\alpha} f_{0}(x, y) d x+\int_{0}^{y+\alpha} f_{1}(x, y) d x=\frac{1}{B_{2}} \\
& \text { i.e. } \int_{0}^{y+\alpha} f_{1}(x, y) d x=\frac{1}{B_{2}}-\int_{0}^{y+\alpha} \frac{1}{B_{1} \cdot B_{2}} d x
\end{aligned}
$$

which gives 


$$
\int_{0}^{y+\alpha} f_{1}(x, y) d x=\frac{\bar{\omega}-y}{B_{1} \cdot B_{2}} \text { for } 0<y<\bar{\omega}
$$

It must be noted that the condition in Equation (3.24) on $f_{1}(x, y)$ holds for any $y \in(0, \bar{\omega})$. This region is illustrated as $O D E J$ in Figure 3.6.

Similar integration along the $y$ direction from Figure 3.6 yields,

$$
\int_{0}^{x+\beta} f_{1}(x, y) d y=\frac{\bar{\omega}-x}{B_{1} \cdot B_{2}} \text { for } 0<x<\bar{\omega}
$$

Again, it can be noted that condition on $f_{1}(x, y)$ in Equation (3.25) holds for any $x \in(0, \bar{\omega})$. This region is illustrated as $O H G F$ in Figure 3.6.

Ideally, $f_{1}(x, y)$ should be defined in the region that is common to $O D E J$ and $O H G F$ i.e. over the region denoted by $O H I J$ in Figure 3.6. Furthermore, Equations (3.24) and (3.25) imply that $f_{1}(x, y)$ would take the following form over $0<x, y<\bar{\omega}$

$$
f_{1}(x, y)=C_{1} \cdot(\bar{\omega}-x) \cdot(\bar{\omega}-y)
$$

The constant $C_{1}$ can be evaluated as

$$
\frac{\bar{\omega}-y}{B_{1} \cdot B_{2}}=\int_{0}^{\bar{\omega}} C_{1} \cdot(\bar{\omega}-x) \cdot(\bar{\omega}-y) d x=C_{1} \cdot(\bar{\omega}-y) \cdot \frac{\bar{\omega}^{2}}{2}
$$

which gives 


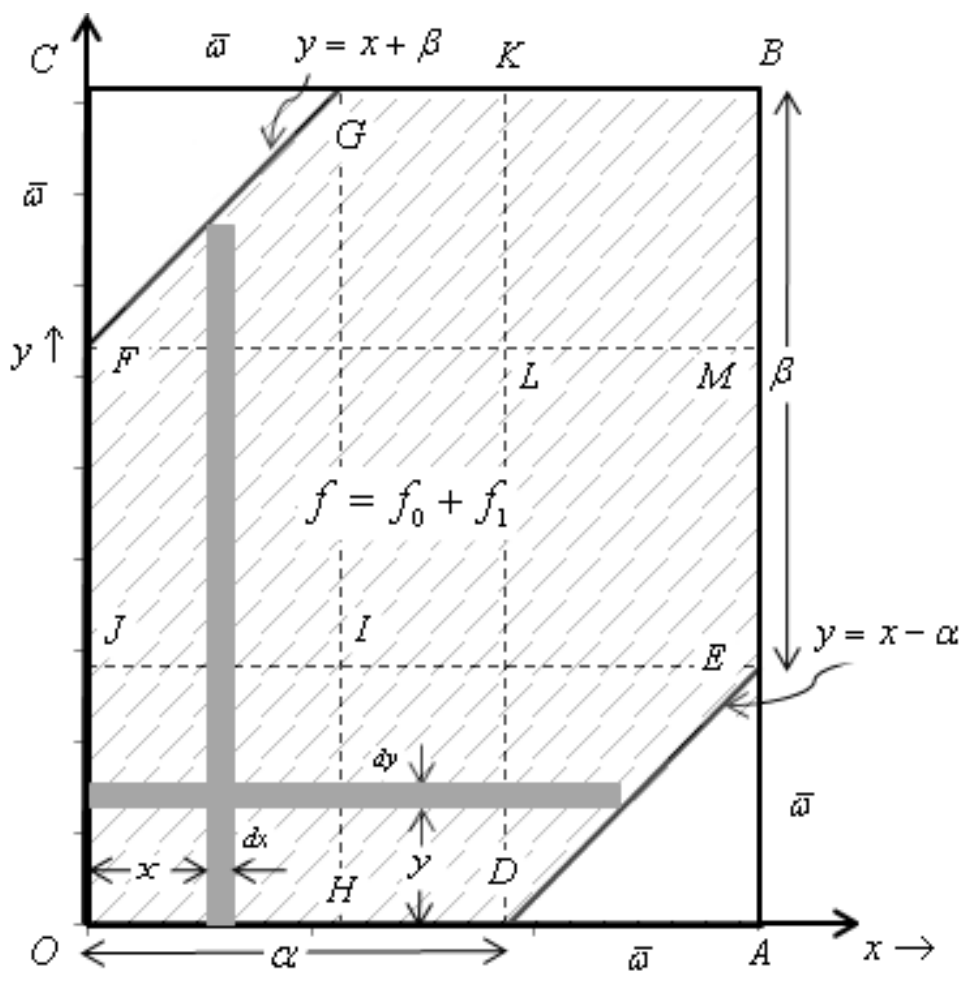

Figure 3.6: Formulation of $f_{1}(x, y)$

$$
C_{1}=\frac{1}{B_{1} \cdot B_{2}} \cdot \frac{2}{\bar{\omega}^{2}}
$$

Equation (3.26) can be rewritten as

$$
f_{1}(x, y)=\frac{1}{B_{1} \cdot B_{2}} \cdot\left(\frac{2 \cdot(\bar{\omega}-x) \cdot(\bar{\omega}-y)}{\bar{\omega}^{2}}\right) \text { for } 0<x, y<\bar{\omega}
$$

The volume under the surface $f_{1}(x, y)$ over $O H I J$ is then given by 


$$
\int_{0}^{\bar{\omega}} \int_{0}^{\bar{\omega}} f_{1}(x, y) d y d x=\frac{\bar{\omega}^{2}}{2 B_{1} \cdot B_{2}}
$$

Notice that this is the half of the additional volume that was intended to be provided by $f_{1}(x, y)$ according to the Equation (3.19). This $f_{1}(x, y)$ within the limits $0<x, y<\bar{\omega}$ makes up for a volume of $\frac{\bar{\omega}^{2}}{2 \cdot B_{1} \cdot B_{2}}$ which is exactly the volume lost due to the constraint $y=x-\alpha$ in the space $D A E$ when integrating with respect to $x$. It is also equal to the volume lost due to the constraint $y=x+\beta$ in the space $F G C$ when integrating with respect to $y$.

By inference and also by central symmetry, we can say that a similar symmetric surface should exist between $x \in\left(\mathrm{B}_{1}-\bar{\omega}, \mathrm{B}_{1}\right)$ and $y \in\left(\mathrm{B}_{2}-\bar{\omega}, \mathrm{B}_{2}\right)$ i.e. region $L M B K$ of Figure 3.6.

Since $f_{1}(x, y)$ is centrally symmetric, we have

$$
f_{1}(x, y)=f_{1}\left(B_{1}-x, B_{2}-y\right)
$$

We can write the expressions for $f_{1}(x, y)$ when $(x, y) \in L M B K$ space using Equation (3.31) as

$$
\begin{aligned}
f_{1}(x, y)=f_{1}\left(B_{1}-x, B_{2}-y\right) & \\
= & \frac{1}{\mathrm{~B}_{1} \cdot \mathrm{B}_{2}} \cdot\left(\frac{2 \cdot\left(\bar{\omega}-\left(B_{1}-x\right)\right) \cdot\left(\bar{\omega}-\left(B_{2}-y\right)\right)}{\bar{\omega}^{2}}\right)
\end{aligned}
$$


Noting that the region $L M B K$ can also be denoted as $\alpha<x<\mathrm{B}_{1}$ and $\beta<y<\mathrm{B}_{2}$, Equation (3.32) can be simplified to rewrite $f_{1}(x, y)$ in this space as

$$
f_{1}(x, y)=\frac{1}{\mathrm{~B}_{1} \cdot \mathrm{B}_{2}} \cdot\left(\frac{2 \cdot(x-\alpha) \cdot(y-\beta)}{\bar{\omega}^{2}}\right) \text { for } \alpha<x<\mathrm{B}_{1} \text { and } \beta<y<\mathrm{B}_{2}
$$

Again,

$$
\int_{\alpha}^{\mathrm{B}_{1}} \int_{\beta}^{\mathrm{B}_{2}} f_{1}(x, y) d y d x=\frac{\bar{\omega}^{2}}{2 \cdot \mathrm{B}_{1} \cdot \mathrm{B}_{2}}
$$

As before, this is exactly the volume lost due to the constraints $y=x-\alpha$ when integrating with respect to $y$. It is also equal to the volume lost due to constraint $y=x+\beta$ when integrating with respect to $x$.

So, the function $f_{1}(x, y)$ can now be defined completely as:

$$
\begin{aligned}
& f_{1}(x, y) \\
& =\left\{\begin{array}{c}
\frac{1}{\mathrm{~B}_{1} \cdot \mathrm{B}_{2}} \cdot\left(\frac{2 \cdot(\bar{\omega}-x) \cdot(\bar{\omega}-y)}{\bar{\omega}^{2}}\right), \quad 0<x, y<\bar{\omega} \\
\frac{1}{\mathrm{~B}_{1} \cdot \mathrm{B}_{2}} \cdot\left(\frac{2 \cdot(x-\alpha) \cdot(y-\beta)}{\bar{\omega}^{2}}\right), \quad \alpha<x<\mathrm{B}_{1} \text { and } \beta<y<\mathrm{B}_{2} \\
0, \text { elsewhere }
\end{array}\right.
\end{aligned}
$$

Figure 3.7(b) shows $f(x, y)$ and the contributions of $f_{0}(x, y)$ and $f_{1}(x, y)$ in various regions. The dotted region in the Figure 3.7(a) represents $f_{1}(x, y)$ as formulated above. Notice, that 
$f_{1}(x, y)$ has non-zero values only in the dotted regions. Elsewhere, it is zero because there is no volume 'lost' due to the applied constraints.

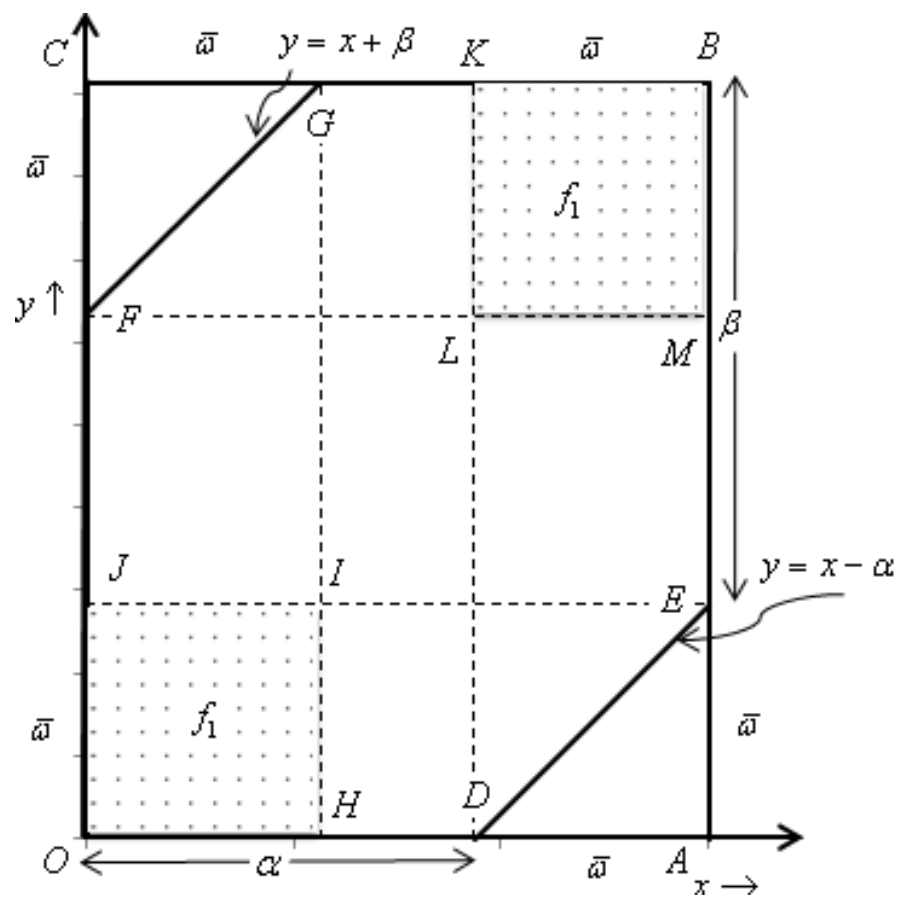

Figure 3.7(a): $f_{1}(x, y)$ 


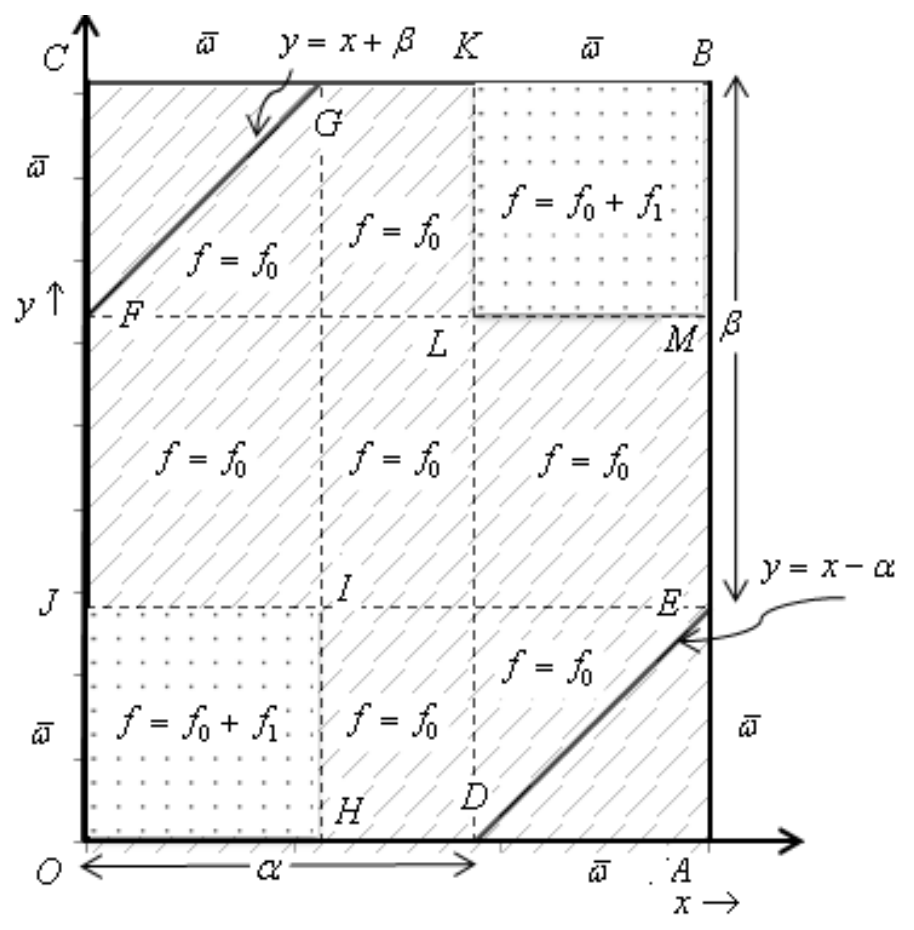

Figure 3.7(b): $f_{X, Y}(x, y)$

So, the joint probability density function $f(x, y)$ can be interpreted as being composed of 2 layers: layer- 0 representing $f_{0}(x, y)$ and layer- 1 representing $f_{1}(x, y)$. The former layer has a density as if the two random variables are independent, and the latter layer 'makes up' for the lost volume in the former layer.

It must also be noted that surface $f_{1}(x, y)$ needs an area of $\bar{\omega} \mathrm{x} \bar{\omega}=\bar{\omega}^{2}$ in the space $O H I J$ and $L M B K$. Many times this may not be possible. We can easily see from the Figure 
3.7 (b) that the above formulation is valid only when $\alpha \geq \bar{\omega}$. The above formulation is NOT valid if $\alpha<\bar{\omega}$. This condition can be written as

$$
\begin{gathered}
B_{1}-\bar{\omega}<\bar{\omega} \\
B_{1}<2 \bar{\omega} \text { i.e. } \mu_{2}<1.176 \mu_{1}
\end{gathered}
$$

which implies that if $\mu_{2}$ is $17.6 \%$ or closer to $\mu_{1}$, the formulation of the joint probability density would not be accurate.

A schematic representation of the sampling space when $\alpha<\bar{\omega}$ can be visualized as shown in Figure 3.8.

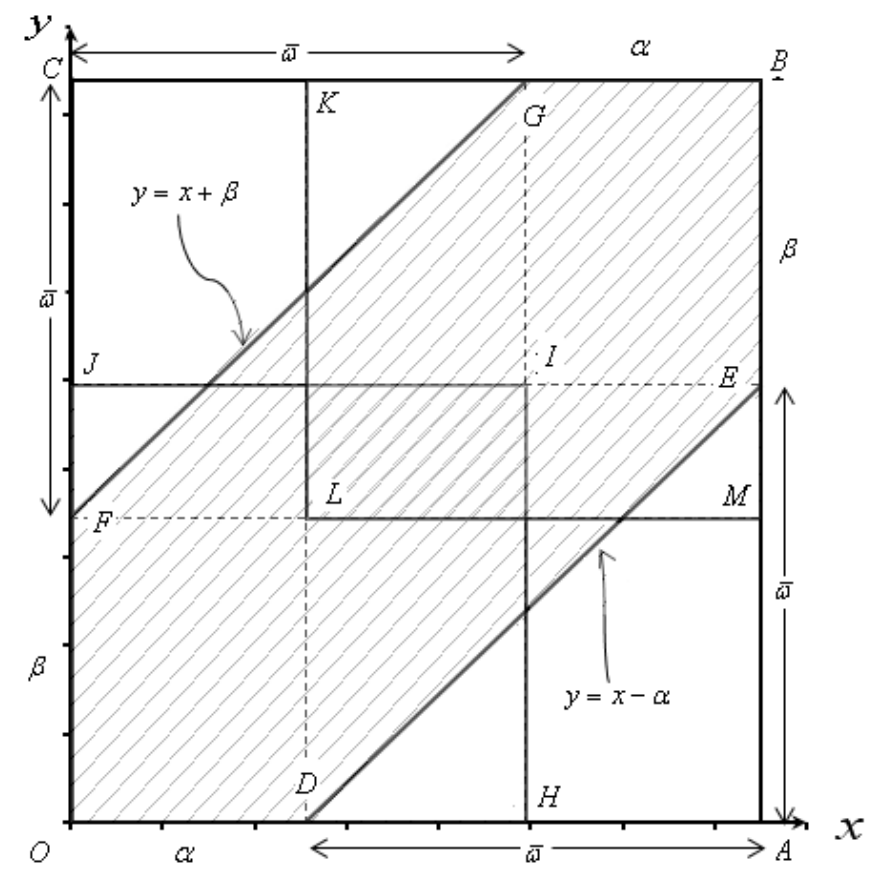

Figure 3.8: The sample space when $\alpha<\bar{\omega}$ 
We can see from the Figure 3.8 that the constraint $y=x-\alpha$ can cut into the space OHIJ (of the dimensions $\bar{\omega} \times \bar{\omega}$ ) needed to define $f_{1}(x, y)$, implying that the volume under the surface would be less than $\frac{\bar{\omega}^{2}}{2 \cdot \mathrm{B}_{1} \cdot \mathrm{B}_{2}}$. Consequently, we need to again 'make up' for the 'lost' volume of $f_{1}(x, y)$ by trying to add another layer - 'Layer-2', in the same manner as we added 'Layer-1' to make up for the lost volume of 'Layer-0'.

Once again, we invoke central symmetry for the between regions $O H I J$ and $K L M B$. To facilitate the illustration for adding Layer-2, the region $O H I J$ is shown by itself in Figure 3.9. Let the Layer-2 be represented by $f_{2}(x, y)$.

Noting the similarity between Figure 3.9 and Figure 3.6, we impose another constraint $y=x+\alpha$ (line $Q R$ ) to achieve symmetry. Notice that the imposed constraint $y=x+\alpha$ exists only for $f_{1}(x, y)$ implying that for this case $-f_{1}(x, y)=0$ if $y>x+\alpha$ as well.

We can then write,

$$
\begin{aligned}
\int_{y+\alpha}^{\bar{\omega}} f_{1}(x, y) d x & =\int_{y+\alpha}^{\bar{\omega}} \frac{1}{\mathrm{~B}_{1} \cdot \mathrm{B}_{2}} \cdot\left(\frac{2 \cdot(\bar{\omega}-x) \cdot(\bar{\omega}-y)}{\bar{\omega}^{2}}\right) d x \\
& =\frac{1}{\mathrm{~B}_{1} \cdot \mathrm{B}_{2}} \cdot \frac{(\bar{\omega}-y) \cdot(\bar{\omega}-y-\alpha)^{2}}{\bar{\omega}^{2}} \\
& =\int_{0}^{y+\alpha} f_{2}(x, y) d x \text { for } 0<y<\bar{\omega}-\alpha
\end{aligned}
$$




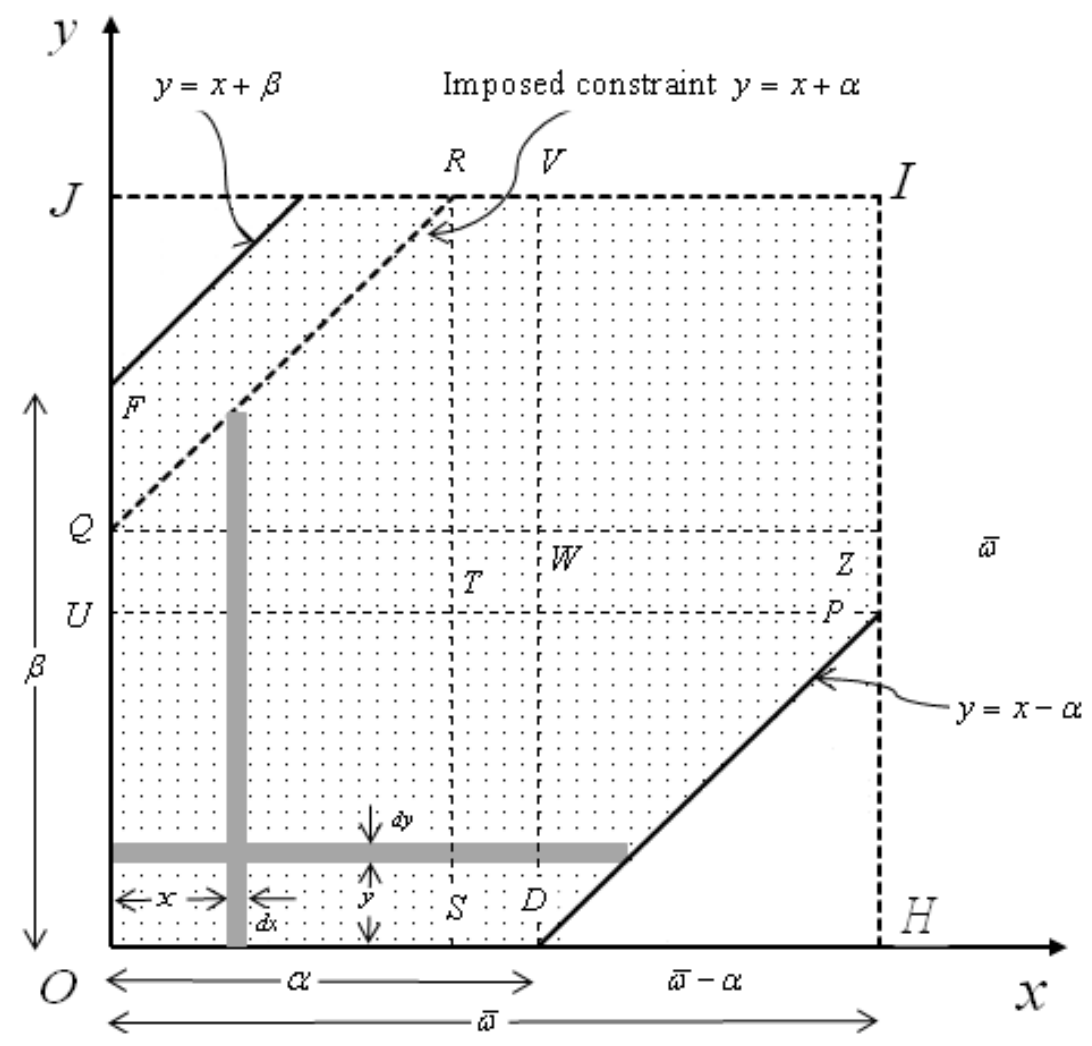

Figure 3.9: Space $O H I J$ when $\alpha<\bar{\omega}$

Similarly, we can get the integration volume lost while integrating in the y-direction as 


$$
\begin{aligned}
\int_{x+\alpha}^{\bar{\omega}} f_{1}(x, y) d y & =\int_{x+\alpha}^{\bar{\omega}} \frac{1}{\mathrm{~B}_{1} \cdot \mathrm{B}_{2}} \cdot\left(\frac{2 \cdot(\bar{\omega}-x) \cdot(\bar{\omega}-y)}{\bar{\omega}^{2}}\right) d y \\
& =\frac{1}{\mathrm{~B}_{1} \cdot \mathrm{B}_{2}} \cdot \frac{(\bar{\omega}-x) \cdot(\bar{\omega}-x-\alpha)^{2}}{\bar{\omega}^{2}} \\
& =\int_{0}^{x+\alpha} f_{2}(x, y) d y \text { for } 0<x<\bar{\omega}-\alpha
\end{aligned}
$$

From Equations (3.37) and (3.38), $f_{2}(x, y)$ for $0<x, y<\bar{\omega}-\alpha$ takes the form:

$$
f_{2}(x, y)=C_{2} \cdot(\bar{\omega}-x) \cdot(\bar{\omega}-y) \cdot(\bar{\omega}-x-\alpha)^{2} \cdot(\bar{\omega}-y-\alpha)^{2}
$$

It can be shown that the constant $C_{2}$ can be evaluated in the same way the constant $C_{1}$ was evaluated in Equation (3.27) i.e.

$$
C_{2}=\frac{1}{\mathrm{~B}_{1} \cdot \mathrm{B}_{2}} \cdot\left(\frac{12}{\bar{\omega}^{2} \cdot(\bar{\omega}-\alpha)^{3} \cdot(3 \bar{\omega}+\alpha)}\right)
$$

This gives,

$$
\begin{gathered}
f_{2}(x, y)=\frac{1}{\mathrm{~B}_{1} \cdot \mathrm{B}_{2}} \cdot\left(\frac{12}{\bar{\omega}^{2} \cdot(\bar{\omega}-\alpha)^{3} \cdot(3 \bar{\omega}+\alpha)}\right) \cdot(\bar{\omega}-x) \cdot(\bar{\omega}-y) \cdot(\bar{\omega}-x-\alpha)^{2} \cdot(\bar{\omega}- \\
y-\alpha)^{2} \text { for } 0<x, y<\bar{\omega}-\alpha
\end{gathered}
$$

Next, symmetry is used to define $f_{2}(x, y)$ for the region $\alpha<x, y<\bar{\omega}$. The Figure 3.10 illustrates both the regions of $f_{2}(x, y)$. Figure 3.10 is identical to Figure 3.6. Figure 3.11 illustrates the contributions of various different layers over the region OHIJ. When $\alpha<$ 
$\bar{\omega}$, the region between the lines $y=x+\alpha$ and $y=x+\beta$ would just have $f_{0}(x, y)$ due to the imposed constraint, whereas OSTU and VWZI contain all the 3 layers.

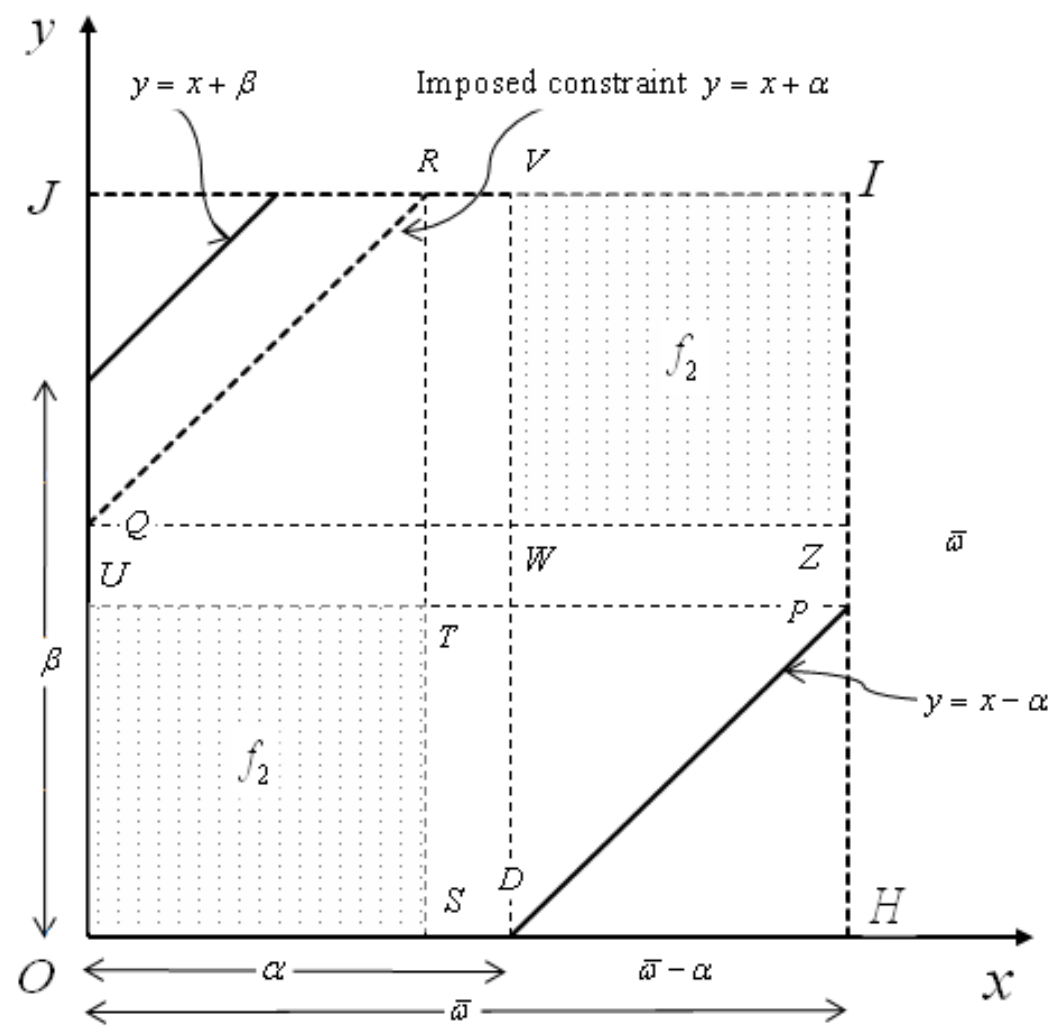

Figure 3.10: $f_{2}(x, y)$ 


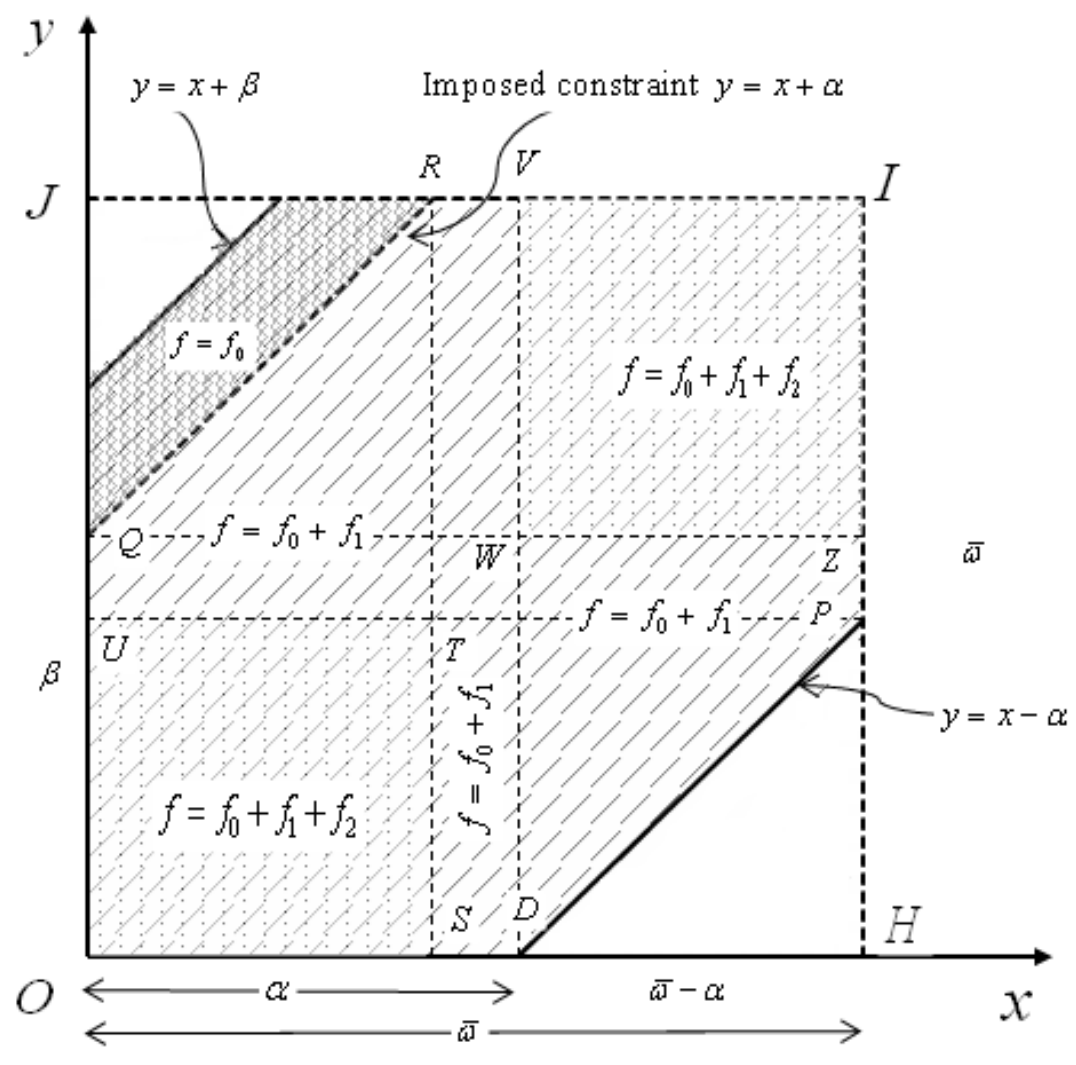

Figure 3.11: $f_{X, Y}(x, y)$ in space $O H I J$ showing various layers when $\alpha<\bar{\omega}$

Now, let us generalize the proposed formulation. To do so, observe the limitation with the formulation i.e. the surface $f_{2}(x, y)$ requires an area of size $(\bar{\omega}-\alpha)^{2}$ (region OSTU of Figure 3.11). This is possible only as long as $\bar{\omega}-\alpha$ is less than $\alpha$ i.e. $\bar{\omega}<2 \alpha$. If $\bar{\omega}>2 \alpha$, another layer $f_{3}(x, y)$ has to be added to make up for the loss in $f_{2}(x, y)$ due to the constraint $y>x-\alpha$. The formulation of $f_{3}(x, y)$ can be developed in a manner similar to that used for $f_{2}(x, y)$ and $f_{1}(x, y)$. 
To generalize, the joint density $f(x, y)$ would have ' $N$ ' such layers over $f_{0}(x, y)$, where ' $N$ ' is the minimum integer such that $\bar{\omega}<N \alpha$. Also, each layer intends to make up for the volume loss of the volume incurred by the previous layer due to the basic constraint $y>$ $x-\alpha$.

$$
\begin{gathered}
\bar{\omega}<N \alpha \\
\text { i.e. } \bar{\omega}<N\left(B_{1}-\bar{\omega}\right) \\
(N+1) \bar{\omega}<N B_{1}
\end{gathered}
$$

Expanding the expressions of $\bar{\omega}$ and $B_{1}$, it can be shown that $N$ layers over $f_{0}(x, y)$, are required if

$$
\mu_{2}<\left[\frac{\frac{1.15}{0.85}-1+N}{N}\right] \mu_{1}
$$

The joint density surface may be completely described as

$$
f_{X, Y}(x, y)=f_{0}(x, y)+\sum_{k=1}^{N} f_{k}(x, y)
$$

Table 3.1 lists the number of layers required to be formulated for $f_{X, Y}(x, y)$ as a function of closeness of $\omega_{1}$ and $\omega_{2}$.

A generic $k^{\text {th }}$ layer shall require an area of size $(\bar{\omega}-(k-1) \alpha)^{2}$ at the origin. The generalized expressions of the $k^{\text {th }}$ layer for $0<x, y<\bar{\omega}-(k-1) \alpha$ can be obtained as 


$$
f_{k}(x, y)=C_{k} \int_{y+\alpha}^{\bar{\omega}-(k-2) \alpha} f_{k-1}(x, y) d x \int_{x+\alpha}^{\bar{\omega}-(k-2) \alpha} f_{k-1}(x, y) d y
$$

where $C_{k}$ is given by

$$
C_{k}=\frac{1}{\int_{0}^{\bar{\omega}-(k-1) \alpha} \int_{x+\alpha}^{\bar{\omega}-(k-2) \alpha} f_{k-1}(x, y) d y d x}
$$

Table 3.1: Number of layers in $f_{X, Y}(x, y)$ as a function of mean frequency ratio

\begin{tabular}{|c|c|}
\hline$\frac{\mu_{2}}{\mu_{1}}<$ & $N$ Layers required \\
\hline 1.353 & 1 \\
\hline 1.176 & 2 \\
\hline 1.118 & 3 \\
\hline 1.088 & 4 \\
\hline 1.070 & 5 \\
\hline 1.059 & 6 \\
\hline
\end{tabular}

It must be duly noted that the necessary conditions of central symmetry within the layer as well as in the entire sample space should be applied to build the entire surface of the $k^{\text {th }}$ layer. The symmetry conditions in $k^{\text {th }}$ layer are summarized in Equation (3.47) below. 


$$
\begin{gathered}
f_{k}(x, y)=f_{k}(\bar{\omega}-(k-2) \alpha-x, \bar{\omega}-(k-2) \alpha-y) \text { for } 0<x, y \\
<\bar{\omega}-(k-2) \alpha \\
f_{k}(x, y)=f_{k}\left(B_{1}-x, B_{2}-y\right)
\end{gathered}
$$

We can see that from the Table 3.1 that the numbers of layers in the complete formulation increase with increasing closeness of the frequencies. For example, if the frequencies are 2\% apart, we can estimate using Equation (3.43) that as many as 17 layers would be required to describe the complete formulation. Formulation of so many layers is not only tedious but also proves to be superfluous. It has been observed that the function $f(x, y)$ described up to a maximum of 2 layers over $f_{0}(x, y)$ and normalizing thereof to obtain the total volume under the surface of $f(x, y)$ to be equal to 1.0 is found to be sufficiently accurate enough to sample the frequencies. The sampling procedure is discussed in detail in section 3.5.

Finally, we can obtain the joint probability density function between the frequencies from:

$$
f_{\Omega_{1}, \Omega_{2}}\left(\omega_{1}, \omega_{2}\right)=f_{X, Y}\left(\omega_{1}-0.85 \mu_{1}, \omega_{2}-0.85 \mu_{2}\right)
$$




\subsection{Plots of the Probability Density Function}

To illustrate the shape of the joint density function $f_{\Omega_{1}, \Omega_{2}}\left(\omega_{1}, \omega_{2}\right)$, four different cases of $\mu_{1}, \mu_{2}$ are considered whose details are given in Table 3.2.

Case 1 is the trivial case with the means of frequencies far apart such that the joint density function can just be described by $f_{0}(x, y)$. Case 2 is selected such that it requires one layer above $f_{0}(x, y)$ while Case 3 is selected such that two layers above $f_{0}(x, y)$ describe the complete distribution function. Case 4 discusses a case where the frequencies are extremely close (2\% apart) where 17 layers are required.

Table 3.2: Different cases considered for plots of the joint probability density function

\begin{tabular}{|c|c|c|c|c|c|}
\hline Case & $\mu_{1}(\mathrm{~Hz})$ & $\mu_{2}(\mathrm{~Hz})$ & $\mu_{2} / \mu_{1}$ & $\bar{\omega}$ & $N$ \\
\hline 1 & 10 & 14 & 1.4 & - & 0 \\
\hline 2 & 10 & 12 & 1.2 & 1.3 & 1 \\
\hline 3 & 10 & 11.4 & 1.14 & 1.81 & 2 \\
\hline 4 & 10 & 10.2 & 1.02 & 2.83 & 17 \\
\hline
\end{tabular}

The Figures 3.12, 3.13 and 3.14 each plot two views of the probability density function $f_{\Omega_{1}, \Omega_{2}}\left(\omega_{1}, \omega_{2}\right)$ for cases 2,3 and 4 respectively. One can see that in cases 2,3 and 4, the red region represents the region of symmetric constraints given by Equations (3.15) and (3.16) indicating $f_{\Omega_{1}, \Omega_{2}}\left(\omega_{1}, \omega_{2}\right)=0$. As the mean of the second frequency $\left(\mu_{2}\right)$ draws 
closer to the mean of the first frequency $\left(\mu_{1}\right)$, the actual sampling space is constrained to a very small region about the diagonal (as for Case 4 in Figure 3.14).

The perspective views of the joint density functions in Figures 3.12, 3.13 and 3.14 show additional layers as they rise from $f_{0}(x, y)$ for cases 2,3 and 4 respectively. The plot of the joint density function in Figure 3.12(b) for Case 2 has two crests rising at the bottom left and top right corners indicating the layer 1 i.e. $f_{1}(x, y)$. Similar crests representing layer 1 can be observed in the perspective views of the density functions for cases 3 and 4 as well. The two small crests rising in the middle of the surface in the plot of the joint density function for Case 3 in Figure 3.13(b) indicate the layer 2. The Figure 3.14(b) of case 4 shows perspective view only up to 2 layers. In this case, the mean frequencies are only $2 \%$ apart. From Table 3.2, 17 layers are required to describe the whole function. From Figure 3.14(a), we can see that the sample space is constrained within a small region about the diagonal. So, instead of computing all the 17 layers (which involves considerable computational effort), it is reasonable to normalize the surface after computation of the first 2 layers. The normalization can be done by simply scaling the surface up to 2 layers such that the total volume under the surface is 1.0 . 


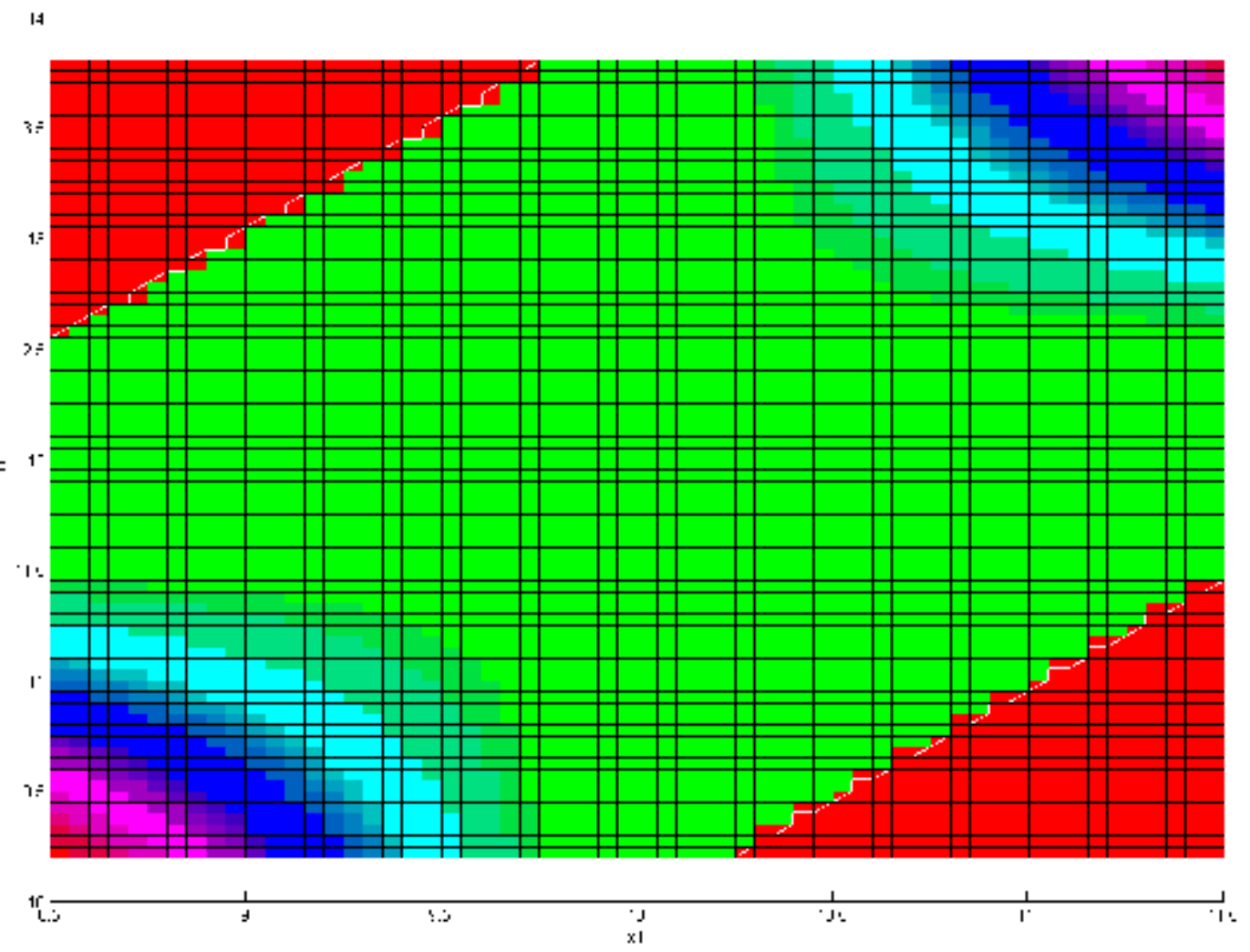

Figure 3.12(a): $f_{\Omega_{1}, \Omega_{2}}\left(\omega_{1}, \omega_{2}\right)$ for case 2 (view of the $\omega_{1}-\omega_{2}$ plane) 


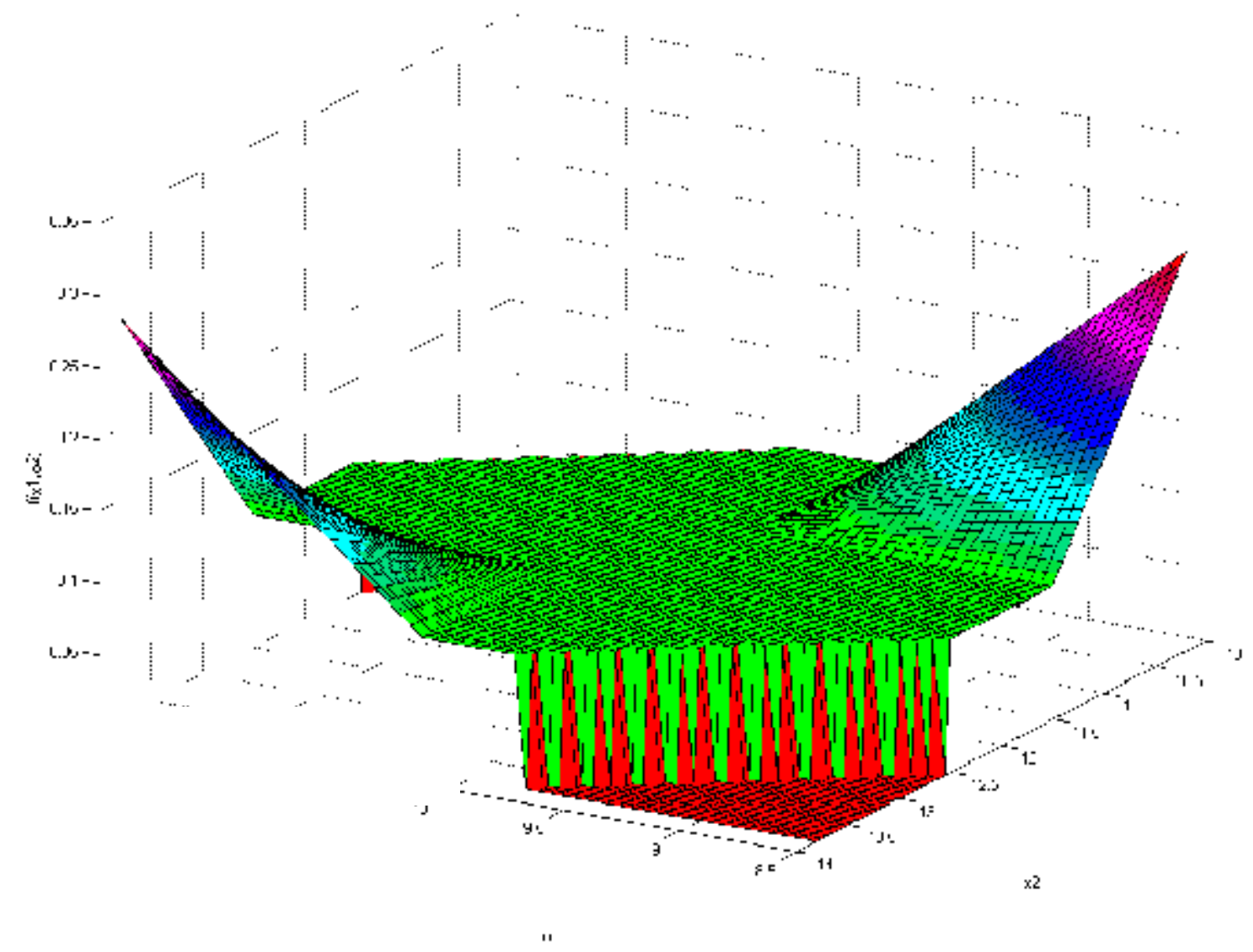

Figure 3.12(b): $f_{\Omega_{1}, \Omega_{2}}\left(\omega_{1}, \omega_{2}\right)$ for case 2 (perspective view) 


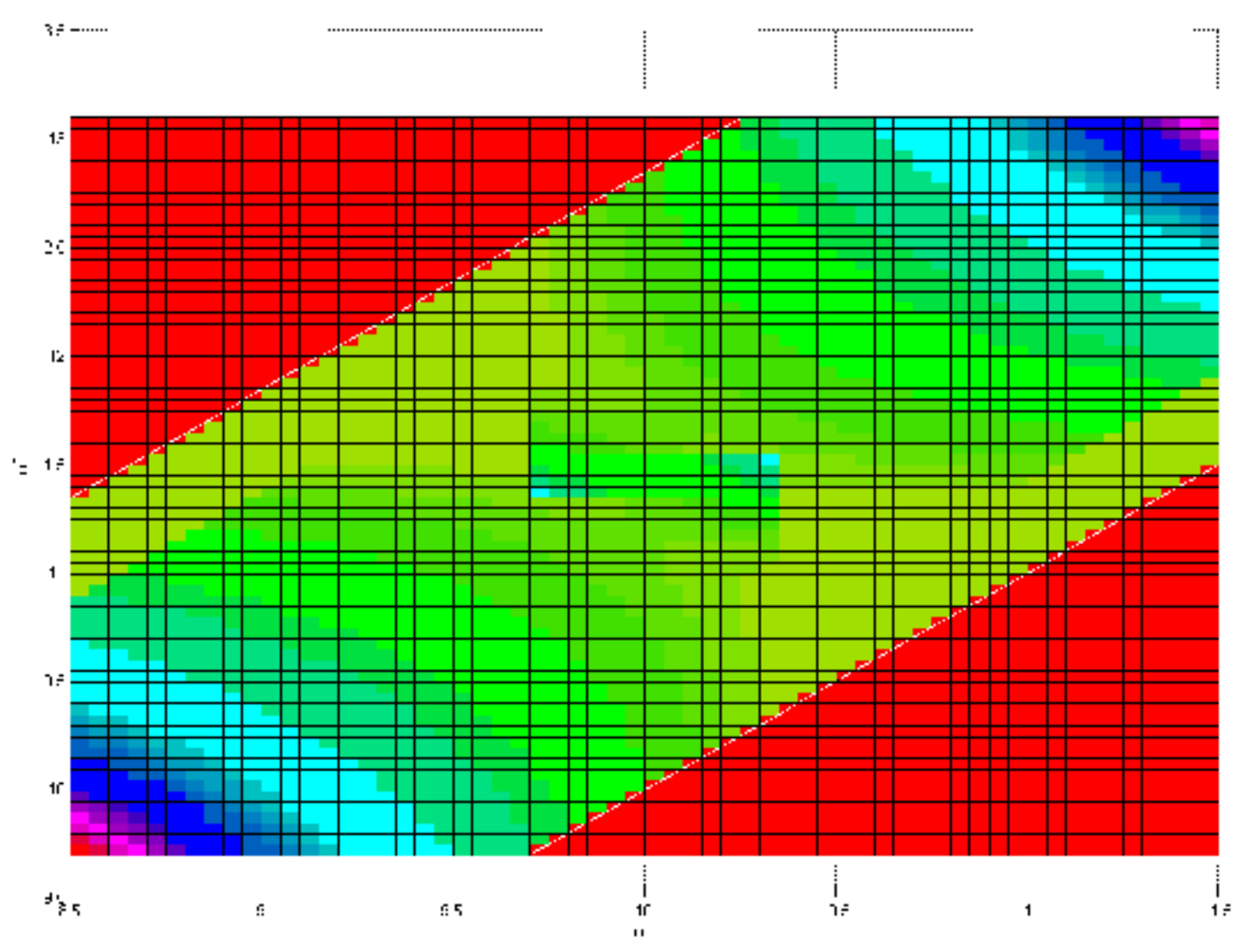

Figure 3.13(a): $f_{\Omega_{1}, \Omega_{2}}\left(\omega_{1}, \omega_{2}\right)$ for case 3 (view of the $\omega_{1}-\omega_{2}$ plane) 


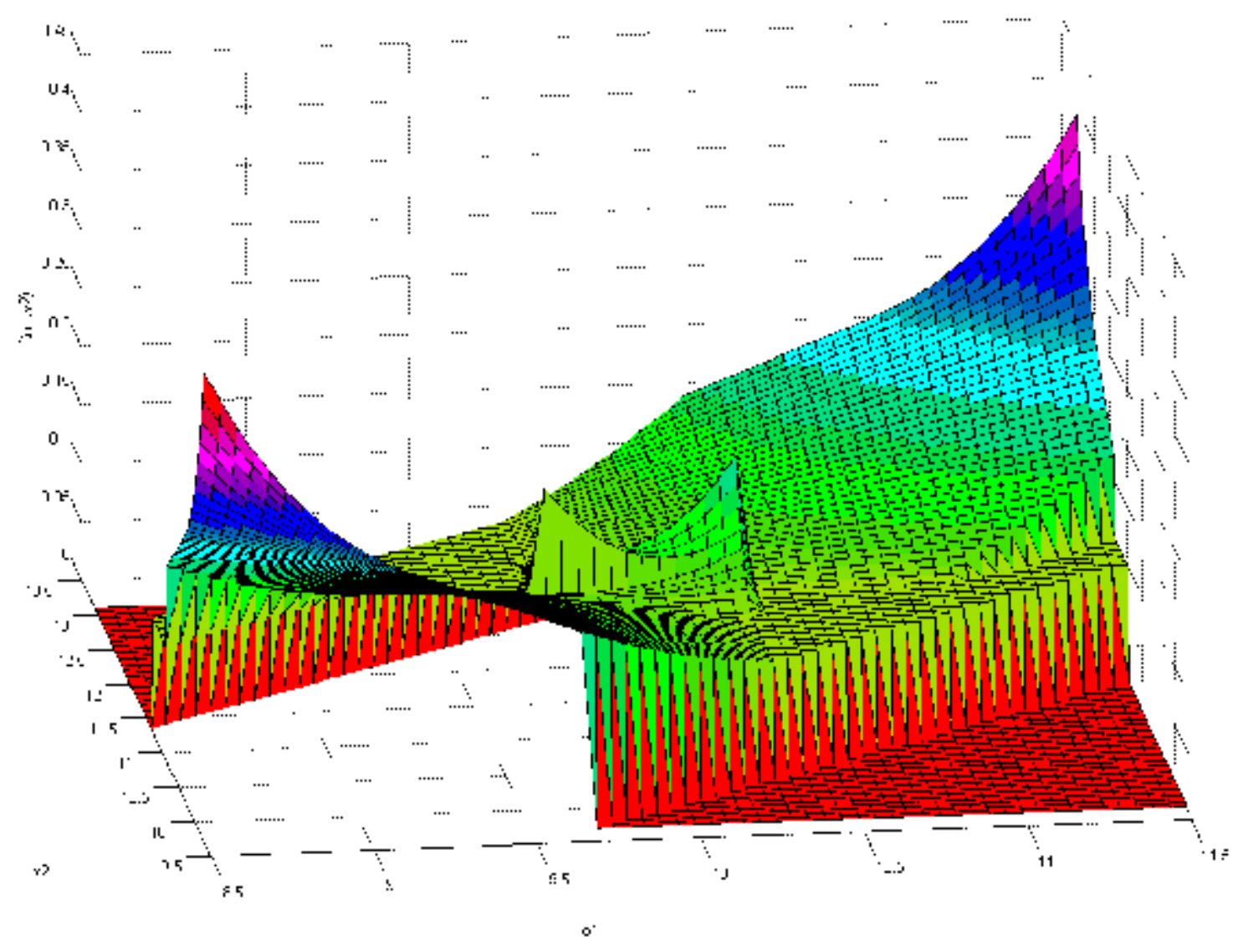

Figure 3.13(b): $f_{\Omega_{1}, \Omega_{2}}\left(\omega_{1}, \omega_{2}\right)$ for case 3 (perspective view) 


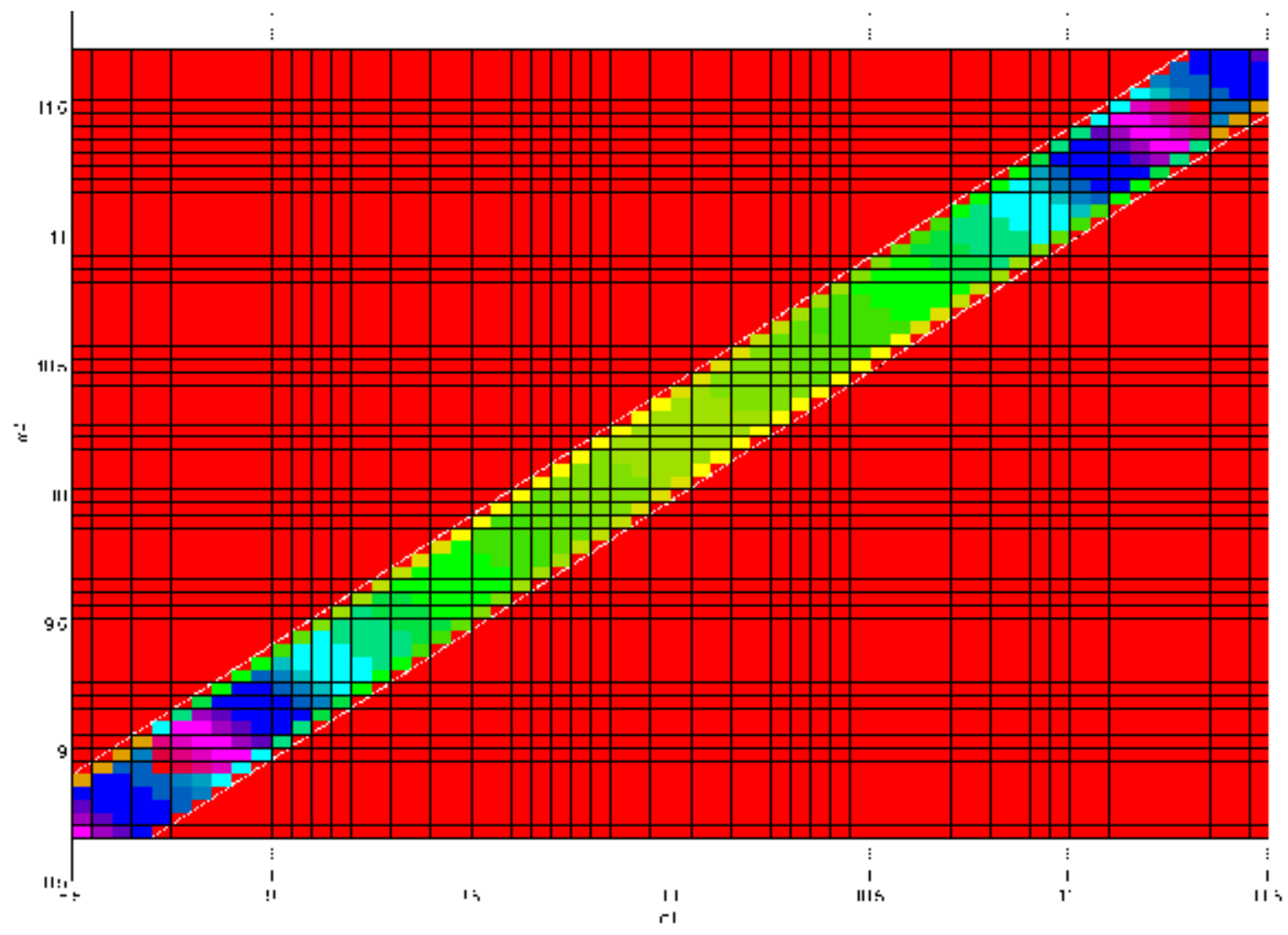

Figure 3.14(a): $f_{\Omega_{1}, \Omega_{2}}\left(\omega_{1}, \omega_{2}\right)$ for case 4 (view of the $\omega_{1}-\omega_{2}$ plane) 


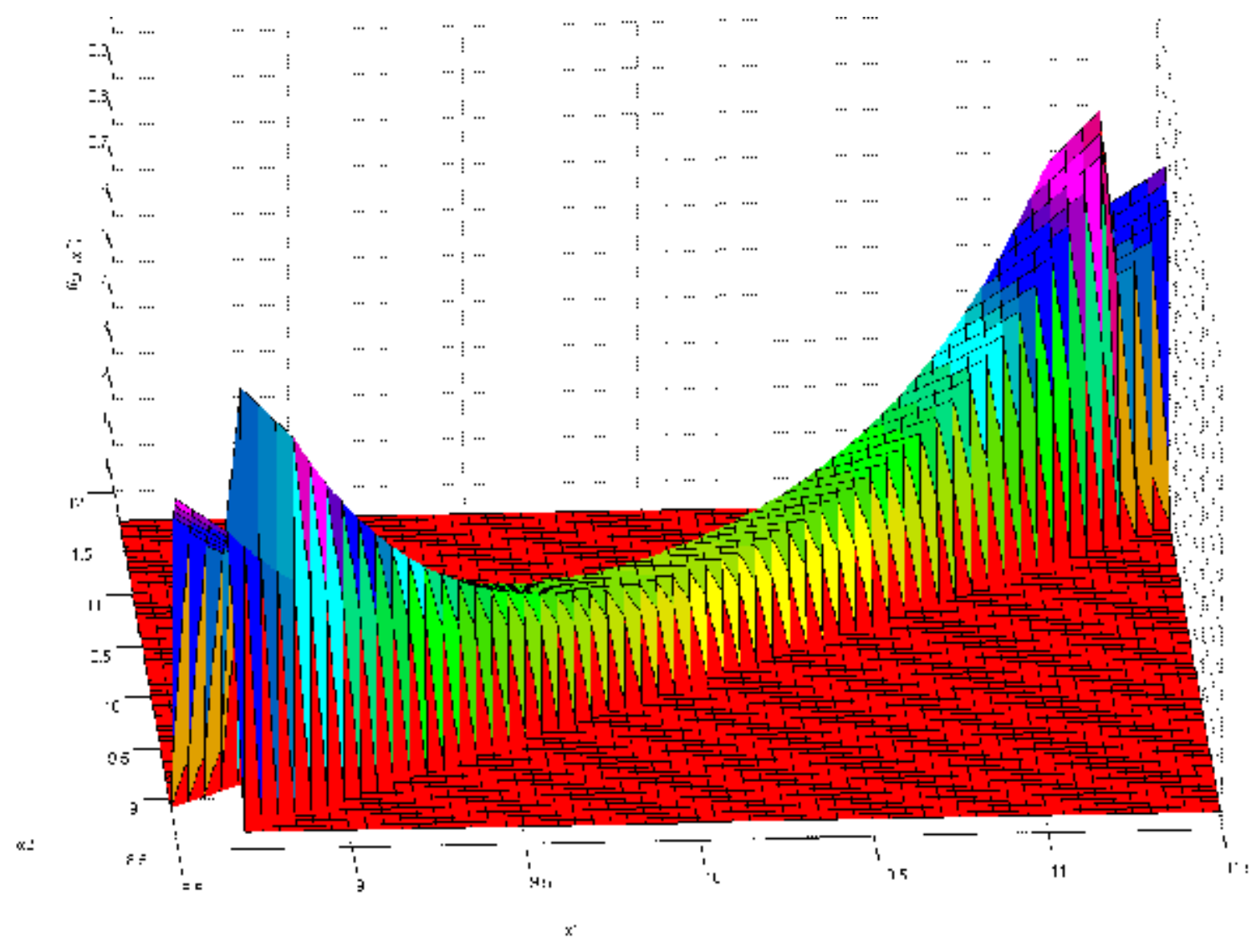

Figure 3.14(b): $f_{\Omega_{1}, \Omega_{2}}\left(\omega_{1}, \omega_{2}\right)$ for case 4 (perspective view up to 2 layers only) 


\subsection{Sampling of Frequencies from Inverse CDF Method}

For a uni-variate random variable $X$ with a continuous cumulative distribution function $(\mathrm{CDF})-F_{X}(X)$ then the random variable $U$ has a uniform distribution between 0 and $1 . U$ can be written as

$$
U=F_{X}(X)
$$

This means that we can generate samples for $X$ given $U$ by

$$
X=F_{X}{ }^{-1}(U)
$$

This technique is sometimes called the inverse CDF method.

The principle can be extended to multivariate distributions (Gentle James, 2005) where if the $\mathrm{CDF}$ of the multivariate random variable $\left(X_{1}, X_{2} \ldots X_{d}\right)$ is decomposed as

$$
\begin{aligned}
& F_{X_{1}, X_{2} \ldots X_{d}}\left(x_{1}, x_{2} \ldots x_{d}\right) \\
& =F_{X_{1}}\left(x_{1}\right) F_{X_{2} \mid X_{1}}\left(x_{2} \mid x_{1}\right) \ldots F_{X_{d} \mid X_{1}, X_{2} \ldots X_{d-1}}\left(x_{d} \mid x_{1}, x_{2} \ldots x_{d-1}\right)
\end{aligned}
$$

And if the functions are invertible, the inverse CDF method is applied sequentially using the independent realizations of $U(0,1)$ random variables $u_{1}, u_{2} \ldots u_{d}$ : 


$$
\begin{gathered}
x_{1}=F_{X_{1}}^{-1}\left(u_{1}\right), \\
x_{2}=F_{X_{2} \mid X_{1}}^{-1}\left(u_{2}\right), \\
\ldots \\
x_{d}=F_{X_{d} \mid X_{1}, X_{2} \ldots X_{d-1}}^{-1}\left(u_{d}\right)
\end{gathered}
$$

The same procedure is used to sample the frequencies from the joint density function formulated in the previous section. We can find the cumulative distribution functions from $f_{\Omega_{1}, \Omega_{2}}\left(\omega_{1}, \omega_{2}\right)$ as

$$
\begin{gathered}
f_{\Omega_{1}}\left(\omega_{1}\right)=\int_{0.85 \mu_{2}}^{1.15 \mu_{2}} f_{\Omega_{1}, \Omega_{2}}\left(\omega_{1}, \omega_{2}\right) d \omega_{2}=\frac{1}{B_{1}} \\
f_{\Omega_{1}, \Omega_{2}}\left(\omega_{1}, \omega_{2}\right)=f_{\Omega_{2} \mid \Omega_{1}}\left(\omega_{2} \mid \omega_{1}\right) \cdot f_{\Omega_{1}}\left(\omega_{1}\right) \\
f_{\Omega_{2} \mid \Omega_{1}}\left(\omega_{2} \mid \omega_{1}\right)=\frac{f_{\Omega_{1}, \Omega_{2}}\left(\omega_{1}, \omega_{2}\right)}{f_{\Omega_{1}}\left(\omega_{1}\right)} \\
F_{\Omega_{1}}\left(\omega_{1}\right)=\int_{0.85 \mu_{1}}^{\omega_{1}} f_{\Omega_{1}}\left(\omega_{1}\right) d \omega_{1} \\
F_{\Omega_{2} \mid \Omega_{1}}\left(\omega_{2} \mid \omega_{1}\right)=\int_{0.85 \mu_{2}}^{\omega_{2}} f_{\Omega_{2} \mid \Omega_{1}}\left(\omega_{2} \mid \omega_{1}\right) d \omega_{2}
\end{gathered}
$$

The functions $f_{\Omega_{2} \mid \Omega_{1}}\left(\omega_{2} \mid \omega_{1}\right)$ and $F_{\Omega_{2} \mid \Omega_{1}}\left(\omega_{2} \mid \omega_{1}\right)$ indicate respectively the conditional probability density function and conditional cumulative distribution function for $\Omega_{2}$ given that $\Omega_{1}=\omega_{1}$. Steps for sampling $\omega_{2}$ from $\omega_{1}$ : 
1. Generate $n$ uniformly distributed random numbers $U_{1}=\left\{u_{11}, \ldots u_{1 i}, \ldots u_{1 n}\right\}$, where $u_{1 i}$ represents the $i^{\text {th }}$ random number.

2. Generate $n$ samples of $\Omega_{1}=\left\{\Omega_{11}, \ldots \Omega_{1 i}, \ldots \Omega_{1 n}\right\}$ by

$$
\Omega_{1 i}=F_{\omega_{1}}^{-1}\left(u_{1 i}\right)
$$

where $\Omega_{1 i}$ is the $i^{\text {th }}$ sample of $\Omega_{1}$.

3. Generate $n$ uniformly distributed random numbers $U_{2}=\left\{u_{21}, \ldots u_{2 i}, \ldots u_{2 n}\right\}$, where $u_{2 i}$ represents the $i^{\text {th }}$ random number of the set $U_{2}$.

4. The $n$ samples of $\Omega_{2}=\left\{\Omega_{21}, \ldots \Omega_{2 i}, \ldots \Omega_{2 n}\right\}$ can be generated from

$$
\Omega_{2 i}=F_{\omega_{2} \mid \omega_{1 i}}^{-1}\left(u_{2 i}\right)
$$

where $\Omega_{2 i}$ is the $i^{\text {th }}$ sample of $\Omega_{2}$.

So, if a structure has $m$ frequencies, the frequencies are generated sequentially. Firstly, $\Omega_{2}$ is sampled using the above detailed steps from the joint density function $f_{\Omega_{1}, \Omega_{2}}\left(\omega_{1}, \omega_{2}\right)$. Having obtained samples for $\Omega_{2}, \Omega_{3}$ is sampled similarly by constructing the distribution $f_{\Omega_{2}, \Omega_{3}}\left(\omega_{2}, \omega_{3}\right)$ and so on. 


\subsection{Illustrative Example for Sampling of Frequencies}

Section 3.5 describes the method for sampling the natural frequencies of a structure by inverse CDF method. This method is used to obtain random sets of natural frequencies in a structure based on the joint distribution function developed in this chapter.

Here, an example of sampling is illustrated for the case 4 described in Table 3.2. A total of 4000 random sample sets were generated. The samples for $\Omega_{1}$ are generated using Latin Hypercube Sampling method. Samples for $\Omega_{2}$ are generated as per the detailed steps given in Section 3.5. The cumulative distributions and their inverses required are computed numerically using a MATLAB code.

Since the constraints are implicitly defined in the formulation of the joint density function, it is always ensured that the random sample sets of frequencies are ordered sets. The sample set of $\Omega_{2}$ passes the Kolmogorov-Smirnov test with confidence limits of 0.03 for uniform distribution between $\pm 15 \%$ of the mean value. Figure 3.15 compares the probability density functions of $\Omega_{2}$ as obtained from sampling with the expected uniform distribution. Figure 3.16 plots the cumulative distribution function of sampled values of $\Omega_{2}$. It can be seen that the obtained probability distribution functions are close to the expected distribution curves.

Thus, the described procedure presents a method to randomly sample the natural frequencies of a structure such that any randomly sampled set is always an ordered set while 
maintaining their individual density functions. Since the joint density functions formulated in this chapter depend only on the mean values of the natural frequencies of a system, the method can be employed for any given set of means of natural frequencies.

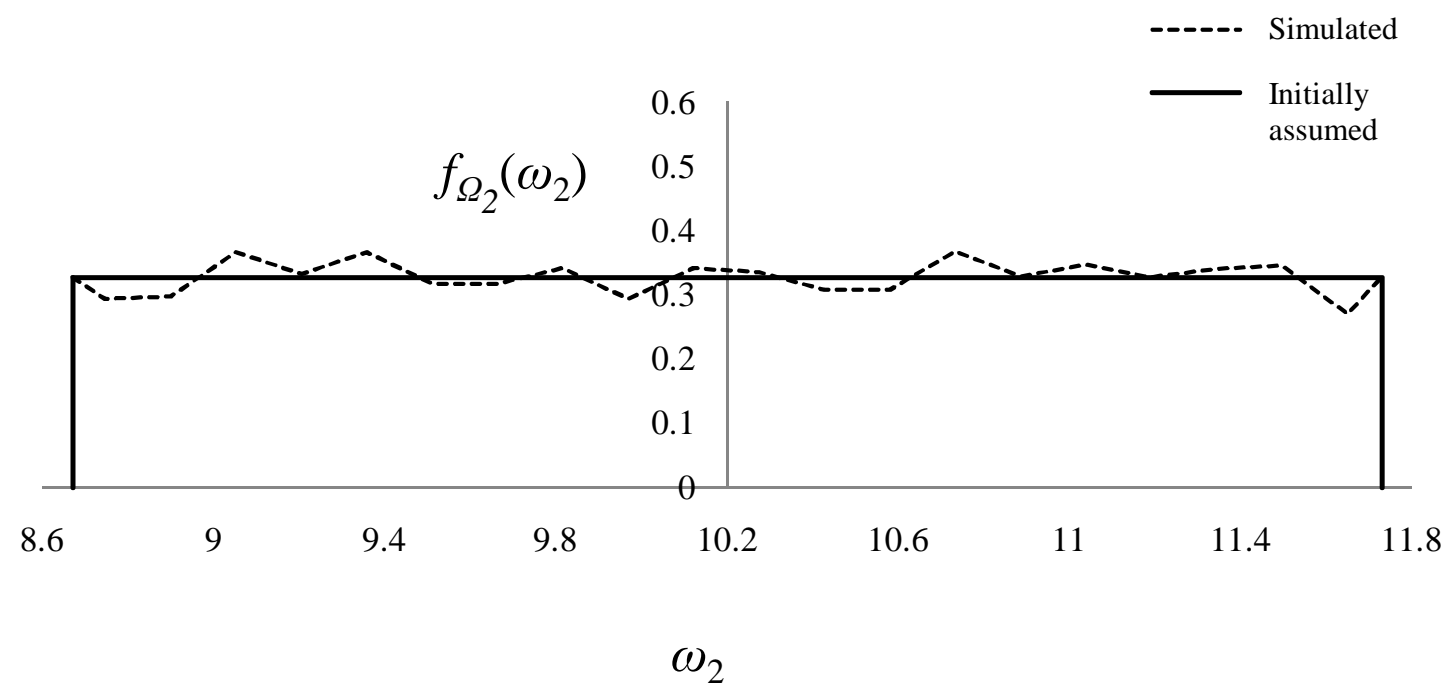

Figure 3.15: Probability density function of sampled $\omega_{2}$ (Case 4) 


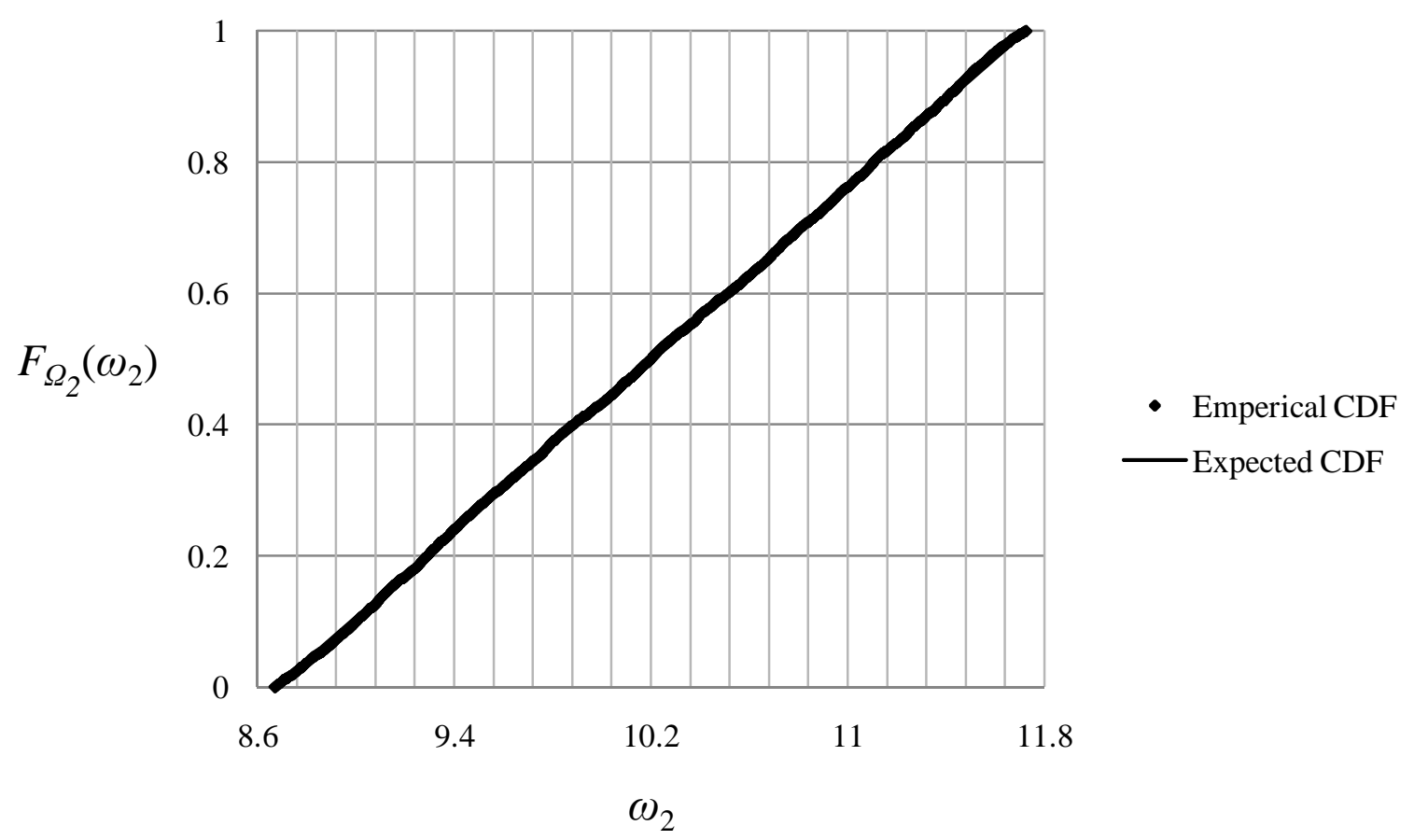

Figure 3.16: Comparison between Empirical CDF and the expected CDF for $\Omega_{2}$ (Case 4) 


\section{CHAPTER - 4 \\ SQUARE ROOT OF MEAN OF SQUARES METHOD OF \\ DETERMINING DESIGN RESPONSE}

\subsection{Design Response}

Gupta and Choi (2005) proposed the Square-Root-of-Mean-of-Squares (SRMS) method to determine the 'design response' of the secondary system. The design response, $R_{0.84}$, is defined as the response corresponding to a non-exceedence probability (NEP) of 0.84 over the set of all possible responses i.e.

$$
P\left[R \leq R_{0.84}\right]=0.84
$$

The design response, $R_{0.84}$, can also be statistically determined as the ' $84^{\text {th }}$ percentile' value (or "mean plus one standard deviation" value for a generic non-Gaussian distribution) from the responses obtained from a Monte Carlo simulation with multiple time history analyses with different sets of sampled natural frequencies, damping ratios of the uncoupled systems and input excitations normalized to the same value of peak ground accelerations (PGA).

Estimating the design response by multiple time history analyses is computationally intensive. Alternatively, the design response $R_{0.84}$ can also be determined by conducting a Monte Carlo simulation with response spectrum. The design spectrum at a nuclear facility is typically defined at a non-exceedence probability of 0.84 over multiple earthquake time 
histories all normalized to a unit PGA to account for the uncertainties in ground motion input. Specifying the design response at a non-exceedence probability of 0.84 over the responses obtained from multiple response spectrum analyses yields values that are excessively conservative as the input spectrum is itself specified at a NEP of 0.84 , and selecting a value corresponding to NEP of 0.84 in response spectrum method is likely to be much higher than the corresponding value from multiple time history analyses.

For a Monte Carlo simulation with response spectrum analysis, we need to generate sets of modal parameters consisting of uncoupled natural frequencies and damping ratios, $z_{i} \equiv\left(\omega_{i}, \xi_{i}\right)$ to account for the uncertainty in modal properties of the system. The individual sets of $z_{i}$ are generated according to the assumed probability criteria for the uncoupled modal parameters of both the primary and secondary systems. For any given $i^{\text {th }}$ set of modal properties $z_{i}$, the response value from a response spectrum analysis using the design spectra is called the "conditional design response" and is denoted by $R_{0.84 / Z_{i}}$. Gupta and Choi (2005) proposed the following two methods based on the conditional responses of the system given a particular set of modal properties.

\subsection{Mean of Conditional Responses}

The design response $R_{0.84}$ according to the mean of conditional responses is given by: 


$$
R_{0.84}=\frac{1}{N} \sum_{i=1}^{N} R_{0.84 / Z_{i}} \Theta_{i}, \quad \Theta_{i}=\frac{1+v_{R}}{1+v_{R / Z_{i}}}
$$

where $N$ is the number of simulated sets of modal properties, $z_{i}, R_{0.84 / z_{i}}$ is the conditional response and $\Theta_{i}$ is a factor dependent upon the coefficient of variation $v_{R}$ for the response $R$ and $v_{R / Z_{i}}$ for the conditional response $R_{0.84 / Z_{i}}$.

If $v_{R} \approx v_{R / Z_{i}}, \Theta_{i}=1$ then we have

$$
R_{0.84} \approx \frac{1}{N} \sum_{i=1}^{N} R_{0.84 / Z_{i}}
$$

\subsection{Square Root of Mean of Squares (SRMS)}

This section presents the detailed formulation for the SRMS method of determining the design response of $R_{0.84}$.

Let us begin by expressing the variance of response $R$

$$
\sigma_{R}^{2}=\frac{\sum_{i=1}^{\mathrm{NT}}\left(R_{i}-\bar{R}\right)^{2}}{N T}
$$


where $\sigma_{R}{ }^{2}$ is the variance of design response; $\bar{R}$ the mean of design response; $R_{i}$ the conditional response for ith set of earthquake and modal properties; NT the total number of responses over all earthquake inputs and modal property sets.

According to the law of conditional expectations,

$$
E[R]=E\left[E\left[R / Z=z_{i}\right]\right]
$$

Equations (4.4) and (4.5) give

$$
\sigma_{R}^{2}=\frac{1}{N T} \sum_{i=1}^{N T}\left[R_{i}-\frac{1}{N S} \sum_{i=1}^{N S} \bar{R}_{Z_{j}}\right]^{2}
$$

where $\bar{R}_{Z_{j}}$ is the mean of conditional response using earthquake inputs for a given $j^{\text {th }}$ set of modal properties; $Z_{j}$ is the $j^{\text {th }}$ set of modal properties and $N S$ is the total number of sets for modal properties.

Next, we can simplify the following expression 


$$
\begin{gathered}
{\left[R_{i}-\frac{1}{N S} \sum_{i=1}^{N S} \bar{R}_{Z_{j}}\right]^{2}=R_{i}{ }^{2}-\frac{2}{N S} \sum_{i=1}^{N S} \bar{R}_{Z_{j}}+\frac{1}{N S^{2}}\left(\sum_{i=1}^{N S} \bar{R}_{Z_{j}}\right)^{2}} \\
=R_{i}{ }^{2}-2 R_{i} \sum_{j=1}^{N S} \bar{R}_{Z_{j}}+\sum_{j=1}^{N S} \bar{R}_{Z_{j}}^{2}+2 R_{i} \sum_{j=1}^{N S} \bar{R}_{Z_{j}}-\sum_{j=1}^{N S} \bar{R}_{Z_{j}}^{2} \\
-\frac{2}{N S} \sum_{i=1}^{N S} \bar{R}_{Z_{j}}+\frac{1}{N S^{2}}\left(\sum_{i=1}^{N S} \bar{R}_{Z_{j}}\right)^{2}=A_{i}+B_{i}
\end{gathered}
$$

where $A_{i}$ and $B_{i}$ are given as

$$
\begin{gathered}
A_{i}=R_{i}^{2}-2 R_{i} \sum_{j=1}^{N S} \bar{R}_{Z_{j}}+\sum_{j=1}^{N S} \bar{R}_{Z_{j}}^{2} \\
B_{i}=2 R_{i} \sum_{j=1}^{N S} \bar{R}_{Z_{j}}-\sum_{j=1}^{N S} \bar{R}_{Z_{j}}^{2}-\frac{2}{N S} \sum_{i=1}^{N S} \bar{R}_{Z_{j}}+\frac{1}{N S^{2}}\left(\sum_{i=1}^{N S} \bar{R}_{Z_{j}}\right)^{2}
\end{gathered}
$$

Let us subdivide $N T$ into $N S$ intervals such that $1<N K_{1}<\cdots N K_{i}<\cdots<N K_{N S}$. We can then write 


$$
\begin{aligned}
\frac{1}{N T} \sum_{i=1}^{N T} A_{i}=\frac{1}{N T} \sum_{i=1}^{N T}\left[R_{i}{ }^{2}-2 R_{i} \sum_{j=1}^{N S} \bar{R}_{Z_{j}}+\sum_{j=1}^{N S} \bar{R}_{Z_{j}}^{2}\right] \\
=\frac{1}{N T} \sum_{i=1}^{N K_{1}}\left[R_{i}{ }^{2}-2 R_{i} \sum_{j=1}^{N S} \bar{R}_{Z_{j}}+\sum_{j=1}^{N S} \bar{R}_{Z_{j}}^{2}\right] \\
+\frac{1}{N T} \sum_{i=N K_{1}+1}\left[R_{i}{ }^{2}-2 R_{i} \sum_{j=1}^{N S} \bar{R}_{Z_{j}}+\sum_{j=1}^{N S} \bar{R}_{Z_{j}}^{2}\right]+\cdots \\
+\frac{1}{N T} \sum_{i=N K_{l}+1}^{N K_{l+1}}\left[R_{i}{ }^{2}-2 R_{i} \sum_{j=1}^{N S} \bar{R}_{Z_{j}}+\sum_{j=1}^{N S} \bar{R}_{Z_{j}}^{2}\right]+\cdots+\cdots \\
+\frac{1}{N T} \sum_{i=N K_{N S-2}+1}^{N K_{N S-1}}\left[R_{i}^{2}-2 R_{i} \sum_{j=1}^{N S} \bar{R}_{Z_{j}}+\sum_{j=1}^{N S} \bar{R}_{Z_{j}}^{2}\right] \\
\left.+R_{i}{ }^{2}-2 R_{i} \sum_{j=1}^{N S} \bar{R}_{Z_{j}}+\sum_{j=1}^{N S} \bar{R}_{Z_{j}}^{2}\right]
\end{aligned}
$$

Note that $N K_{N S}=N T$.

Equation (4.9) can be rearranged as 


$$
\begin{aligned}
\frac{1}{N T} \sum_{i=1}^{N T} A_{i}=\frac{1}{N T} \sum_{i=1}^{N K_{1}}\left[\left(R_{i}-\bar{R}_{Z_{1}}\right)^{2}-2 R_{i} \sum_{j=2}^{N S} \bar{R}_{Z_{j}}+\sum_{j=2}^{N S} \bar{R}_{Z_{j}}^{2}\right]+\cdots \\
+\frac{1}{N T} \sum_{i=N K_{N S-1}+1}^{N K_{N S}}\left[\left(R_{i}-\bar{R}_{Z_{N S}}\right)^{2}-2 R_{i} \sum_{j=1}^{N S-1} \bar{R}_{Z_{j}}+\sum_{j=1}^{N S-1} \bar{R}_{Z_{j}}^{2}\right] \\
=\frac{1}{N T}\left\{\sum_{i=1}^{N K_{1}}\left(R_{i}-\bar{R}_{Z_{1}}\right)^{2}-2 \sum_{i=1}^{N K_{1}} R_{i} \sum_{j=2}^{N S} \bar{R}_{Z_{j}}+\sum_{i=1}^{N K_{1}} \sum_{j=2}^{N S} \bar{R}_{Z_{j}}^{2}+\cdots\right. \\
+\sum_{i=N K_{N S-1}+1}^{N K_{N S}}\left(R_{i}-\bar{R}_{Z_{N S}}\right)^{2} \\
\left.-2 \sum_{i=N K_{N S-1}+1}^{N K_{N S}} R_{i}^{N S \sum_{j=2}^{N}} \bar{R}_{Z_{j}}+\sum_{i=N K_{N S-1}+1}^{N K_{N S}} \sum_{j=2}^{N S-1} \bar{R}_{Z_{j}}^{2}\right\}
\end{aligned}
$$

For simplicity, let each interval be equal i.e. $N K_{1}=N K_{2}=\cdots N K_{i}=\cdots=N K_{N S}=K$. Note that $N T=(K)(N S)$. Equation (4.10) can then be simplified as

$$
\begin{gathered}
\frac{1}{N T} \sum_{i=1}^{N T} A_{i}=\frac{1}{N T}\left\{K \sigma_{Z_{1}}^{2}-2 K \bar{R}_{Z_{1}} \sum_{j=2}^{N S} \bar{R}_{Z_{j}}+K \sum_{j=2}^{N S} \bar{R}_{Z_{j}}^{2}+\cdots+K \sigma_{Z_{N S}}^{2}\right. \\
\left.-2 K \bar{R}_{Z_{N S}} \sum_{j=2}^{N S-1} \bar{R}_{Z_{j}}+\sum_{j=2}^{N S-1} \bar{R}_{Z_{j}}^{2}\right\}= \\
\frac{1}{N S}\left\{\sum_{i=1}^{N S} \sigma_{Z_{i}}^{2}-2 \sum_{i=1}^{N S}\left[\bar{R}_{Z_{i}} \sum_{j=1}^{N S} \bar{R}_{Z_{j}}\right]+(N S-1) \sum_{i=1}^{N S} \bar{R}_{Z_{i}}^{2}\right\}
\end{gathered}
$$

Next, we simplify $B_{i}$ in Equation (4.8) following the same procedure as above 


$$
\frac{1}{N T} \sum_{i=1}^{N T} B_{i}=\frac{1}{N S}\left\{\left(2-\frac{1}{N S}\right)\left(\sum_{j=1}^{N S} \bar{R}_{Z_{j}}\right)^{2}-N S \sum_{j=1}^{N S} \bar{R}_{Z_{j}}^{2}\right\}
$$

Substituting Equations (4.7), (4.11) and (4.12) into Equation (4.6), variance $\sigma_{R}^{2}$ can be expressed as

$$
\begin{aligned}
\sigma_{R}^{2}=\frac{1}{N S}\left\{\sum_{i=1}^{N S} \sigma_{Z_{i}}^{2}-2 \sum_{i=1}^{N S}\left[\bar{R}_{Z_{i}} \sum_{j=1}^{N S} \bar{R}_{Z_{j}}\right]+(N S-1) \sum_{i=1}^{N S} \bar{R}_{Z_{i}}^{2}\right\} \\
+\frac{1}{N S}\left\{\left(2-\frac{1}{N S}\right)\left(\sum_{i=1}^{N S} \bar{R}_{Z_{i}}\right)^{2}-N S \sum_{i=1}^{N S} \bar{R}_{Z_{i}}^{2}\right\} \\
=\frac{1}{N S}\left\{\sum_{i=1}^{N S} \sigma_{Z_{i}}^{2}-2 \sum_{i=1}^{N S}\left[\bar{R}_{Z_{i}} \sum_{j=1}^{N S} \bar{R}_{Z_{j}}\right]-\sum_{i=1}^{N S} \bar{R}_{Z_{i}}^{2}\right. \\
\left.+\left(2-\frac{1}{N S}\right)\left(\sum_{i=1}^{N S} \bar{R}_{Z_{i}}\right)^{2}\right\} \\
=\frac{1}{N S}\left\{\sum_{i=1}^{N S} \sigma_{Z_{i}}^{2}+\sum_{i=1}^{N S} \bar{R}_{Z_{j}}^{2}-\frac{1}{N S}\left(\sum_{i=1}^{N S} \bar{R}_{Z_{i}}\right)^{2}\right\}
\end{aligned}
$$

or 


$$
\begin{gathered}
N S \sigma_{R}^{2}=\sum_{i=1}^{N S} \sigma_{Z_{i}}^{2}+\sum_{i=1}^{N S} \bar{R}_{Z_{j}}^{2}-\frac{1}{N S}\left(\sum_{j=1}^{N S} \bar{R}_{Z_{j}}\right)^{2} \\
=\sum_{i=1}^{N S} \sigma_{Z_{i}}^{2}+\sum_{i=1}^{N S} \bar{R}_{Z_{j}}^{2}-N S \bar{R}^{2} \\
=\sum_{i=1}^{N S}\left(\sigma_{Z_{i}}^{2}+2 \sigma_{Z_{i}} \bar{R}_{Z_{i}}+\bar{R}_{Z_{j}}^{2}\right)-2 \sum_{i=1}^{N S} \sigma_{Z_{i}} \bar{R}_{Z_{i}}-N S \bar{R}^{2} \\
N S\left(\sigma_{R}^{2}+\bar{R}^{2}\right)=\sum_{i=1}^{N S} S_{Z_{i}}^{2}-2 \sum_{i=1}^{N S} \sigma_{Z_{i}} \bar{R}_{Z_{i}}
\end{gathered}
$$

where $S_{Z_{i}}$ is the mean plus one standard deviation for $i^{\text {th }}$ set of modal properties.

Let us add $N S\left(2 \sigma_{R} \bar{R}\right)$ to both sides of Equation (4.15) and rearrange it.

$$
\begin{aligned}
& N S\left(\sigma_{R}^{2}+\bar{R}^{2}+2 \sigma_{R} \bar{R}\right)=\sum_{i=1}^{N S} S_{Z_{i}}^{2}-2 \sum_{i=1}^{N S} \sigma_{Z_{i}} \bar{R}_{Z_{i}}+2 N S \sigma_{R} \bar{R} \\
& S^{2}=\left(\sigma_{R}+\bar{R}\right)^{2}=\frac{1}{N S}\left(\sum_{i=1}^{N S} S_{Z_{i}}^{2}-2 \sum_{i=1}^{N S} \sigma_{Z_{i}} \bar{R}_{Z_{i}}+2 N S \sigma_{R} \bar{R}\right)
\end{aligned}
$$

Further if $v_{R}$ is the coefficient of variation for $R, \sigma_{R} \bar{R}$ can be expressed by

$$
\sigma_{R} \bar{R}=\left[\frac{1}{\beta}\left(\sigma_{R}+\bar{R}\right)\right]^{2} ; v_{R}=\frac{\sigma_{R}}{\bar{R}}
$$




$$
\begin{gathered}
\bar{R}^{2}=\frac{\bar{R}^{2}}{\beta^{2}}\left(1+v_{R}\right)^{2} \\
\frac{1}{\beta^{2}}=\frac{v_{R}}{\left(1+v_{R}\right)^{2}}
\end{gathered}
$$

where $\beta$ is a constant. Substituting Equation (4.20) into (4.18), we can write

$$
\begin{gathered}
\sigma_{R} \bar{R}=\left[\frac{\sqrt{v_{R}}}{\left(1+v_{R}\right)}\left(\sigma_{R}+\bar{R}\right)\right]^{2}=\frac{v_{R}}{\left(1+v_{R}\right)^{2}}\left(\sigma_{R}+\bar{R}\right)^{2} \\
=\frac{v_{R}}{\left(1+v_{R}\right)^{2}} S^{2}
\end{gathered}
$$

Similarly, $\sigma_{Z_{i}} \bar{R}_{Z_{i}}$ can be written as

$$
\sigma_{Z_{i}} \bar{R}_{Z_{i}}=\frac{v_{Z_{i}}}{\left(1+v_{Z_{i}}\right)^{2}} S_{Z_{i}}^{2}, \quad v_{Z_{i}}=\frac{\sigma_{Z_{i}}}{\bar{R}_{Z_{i}}}
$$

Equations (4.17), (4.21) and (4.22) give

$$
S^{2}=\frac{1}{N S}\left(\sum_{i=1}^{N S} S_{Z_{i}}^{2}-2 \sum_{i=1}^{N S} \frac{v_{Z_{i}}}{\left(1+v_{Z_{i}}\right)^{2}} S_{Z_{i}}^{2}+2 N S \frac{v_{R}}{\left(1+v_{R}\right)^{2}} S^{2}\right)
$$

Equation (4.23) upon simplification gives 


$$
S^{2}=\frac{1}{N S} \sum_{i=1}^{N S} S_{Z_{i}}^{2} \frac{\left(1-{ }^{2 v_{Z_{i}}} /\left(1+v_{Z_{i}}\right)^{2}\right)}{\left(1-{ }^{2 v_{R}} /\left(1+v_{R}\right)^{2}\right)}
$$

Finally, the response corresponding to mean plus one standard deviation can be obtained by

$$
S=\left[\frac{1}{N S} \sum_{i=1}^{N S} S_{Z_{i}}^{2} \theta_{Z_{i}}\right]^{1 / 2}, \theta_{Z_{i}}=\frac{\left(1-{ }^{2 v_{Z_{i}}} /\left(1+v_{Z_{i}}\right)^{2}\right)}{\left(1-2 v_{R} /\left(1+v_{R}\right)^{2}\right)}
$$

The expression for $R_{0.84}$ is given by

$$
\begin{aligned}
& R_{0.84}=\left[\frac{1}{N} \sum_{i=1}^{N} R_{0.84 / Z_{i}}^{2} \Theta_{i}\right]^{1 / 2} \\
& \Theta_{i}=\left(\frac{1-{ }^{2 v_{R / Z_{i}}} /\left(1+v_{R / Z_{i}}\right)^{2}}{1-{ }^{2 v_{R}} /\left(1+v_{R}\right)^{2}}\right)
\end{aligned}
$$

where $N$ is the number of simulated sets of modal properties, $z_{i}, R_{0.84 / Z_{i}}$ is the conditional response obtained as the response value from the analysis using the $i^{\text {th }}$ modal property set and $\Theta_{i}$ is a factor dependent upon the coefficient of variation $v_{R}$ for the response $R$ and coefficient of variation $v_{R / Z_{i}}$ for the conditional response $R_{0.84 / Z_{i}}$. 
Again, with the assumption that $\Theta_{i}=1$, Equation (4.26) simplifies as

$$
R_{0.84} \approx\left[\frac{1}{N} \sum_{i=1}^{N} R_{0.84 / Z_{i}}{ }^{2}\right]^{1 / 2}
$$

In Gupta and Choi (2005), four SDOF primary - SDOF secondary systems with various degrees of tuning between them were considered. The natural frequencies and damping ratios of both primary and secondary systems were assumed to be independent Gaussian random variables with a coefficient of variation of 0.15 . For each coupled system, a total of 7500 secondary system responses using time history analysis are evaluated considering combinations of 75 real earthquakes records normalized to the same value of peak ground acceleration (PGA) and 100 sets of randomly sampled frequencies and modal damping ratios.

The design response from multiple response spectrum analyses was calculated according to the two methods described above. These values are compared with the design response values obtained from the time history analyses as the $84^{\text {th }}$ percentile value over the total of 7500 responses. The mean of conditional responses method gave design responses that were consistently a little less than the $84^{\text {th }}$ percentile value from the time history analyses. The SRMS method yielded results that were close to the corresponding results from the time history analysis. 


\section{CHAPTER - 5 \\ NUMERICAL EXAMPLES}

This chapter describes the Task-5 listed under Section 1.4 of this thesis. As mentioned before, the objective of the thesis is focused on verification and application of the SRMS method to MDOF primary - MDOF secondary systems. Numerical examples are presented here to illustrate the same.

\subsection{Relative Significance of Uncertainties in Earthquake Input and Modal Properties}

Gupta and Choi (2005) used SDOF primary - SDOF secondary system to illustrate that uncertainties in frequencies and modal damping ratios can result in significant variation of secondary system responses if the frequencies of the uncoupled systems are tuned or nearly tuned. In this section, a numerical study is conducted to examine the relative importance of uncertainties in ground motion input and uncoupled modal properties in a MDOF primary MDOF secondary system. A simple 4-DOF coupled system is considered as shown in Figure 5.1. This is representative of a simple model of a two-storey building and base-supported equipment. The primary and secondary structures each consist of two massless shear beam elements and two lumped masses. The base of the secondary system is coupled to the lower storey of the primary system. Two different coupled systems of this type are considered for the study. One of these coupled systems comprises of detuned primary and secondary systems while the other coupled system considers the case where the fundamental mode of 
the primary system is tuned with the fundamental mode of the secondary system. Table 5.1 gives the characteristics of two uncoupled systems in each of the two 4-DOF coupled systems.

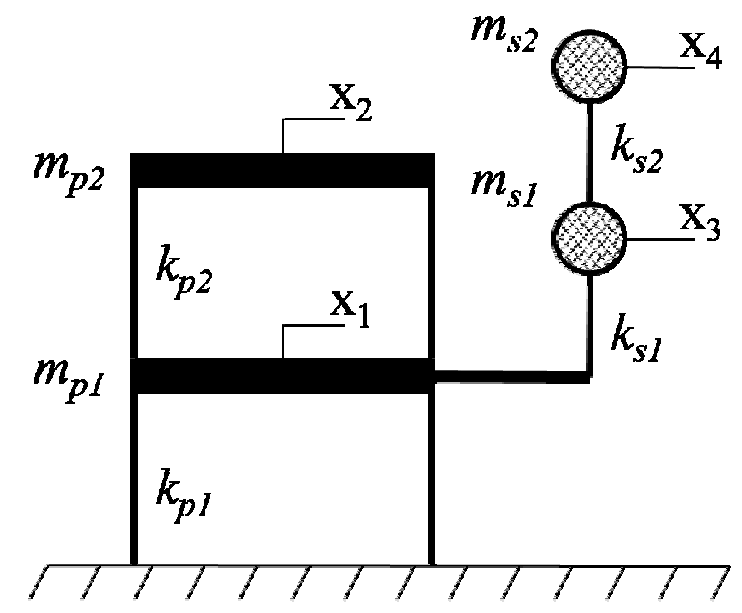

Figure 5.1: The 2 DOF primary - 2 DOF secondary system model

Table 5.1: Modal properties of uncoupled primary and secondary systems

\begin{tabular}{|c|c|c|c|c|c|c|c|}
\hline \multirow{3}{*}{$\begin{array}{c}\text { Model } \\
\#\end{array}$} & \multirow{3}{*}{$\begin{array}{c}\text { Model } \\
\text { Description }\end{array}$} & \multicolumn{3}{|c|}{ PRIMARY SYSTEM } & \multicolumn{3}{|c|}{ SECONDARY SYSTEM } \\
\hline & & \multicolumn{2}{|c|}{$\begin{array}{l}\text { Natural Frequencies } \\
\qquad(\mathrm{Hz})\end{array}$} & \multirow{2}{*}{$\begin{array}{l}\text { Damping } \\
\text { Ratio }\left(\xi_{p}\right)\end{array}$} & \multicolumn{2}{|c|}{$\begin{array}{c}\text { Natural Frequencies } \\
(\mathrm{Hz})\end{array}$} & \multirow{2}{*}{$\begin{array}{c}\text { Damping } \\
\text { Ratio } \\
\left(\xi_{s}\right)\end{array}$} \\
\hline & & $\omega_{p 1}$ & $\omega_{p 2}$ & & $\omega_{s 1}$ & $\omega_{s 2}$ & \\
\hline 1 & Detuned & 0.5 & 1.0 & $6 \%$ & 2.5 & 6.0 & $2 \%$ \\
\hline 2 & $\begin{array}{c}\text { Tuned } \\
\left(\omega_{p 1} \approx \omega_{s 1)}\right.\end{array}$ & 2.4 & 4.8 & $6 \%$ & 2.5 & 6.0 & $2 \%$ \\
\hline
\end{tabular}


The relative effect of uncertainties in earthquake input, frequencies and modal damping ratios of the uncoupled primary and secondary system is evaluated by conducting multiple time history analyses for each of the two coupled systems. To model uncertainty in earthquake input, 75 real earthquake records normalized to the same value of Peak Ground Acceleration (PGA) have been considered (see Appendix - B for characteristics of earthquake records). Each earthquake time history yields one set of secondary system responses. Next, the responses are calculated using only a single normalized earthquake record but considering variations in uncoupled natural frequencies of primary and secondary systems. Finally, the secondary system responses are evaluated by considering a variation in only modal damping ratios of the uncoupled systems. The natural frequencies and damping ratios are assumed to be uniform random variables varying within $\pm 15 \%$ of their respective mean values. Figure 5.2 shows the relative values of displacements of the lower mass $\left(m_{s 1}\right)$ of the secondary system for each of the three types of analyses described above. In the figure, the displacements are normalized with respect to the maximum displacement evaluated in a particular type of analysis. 


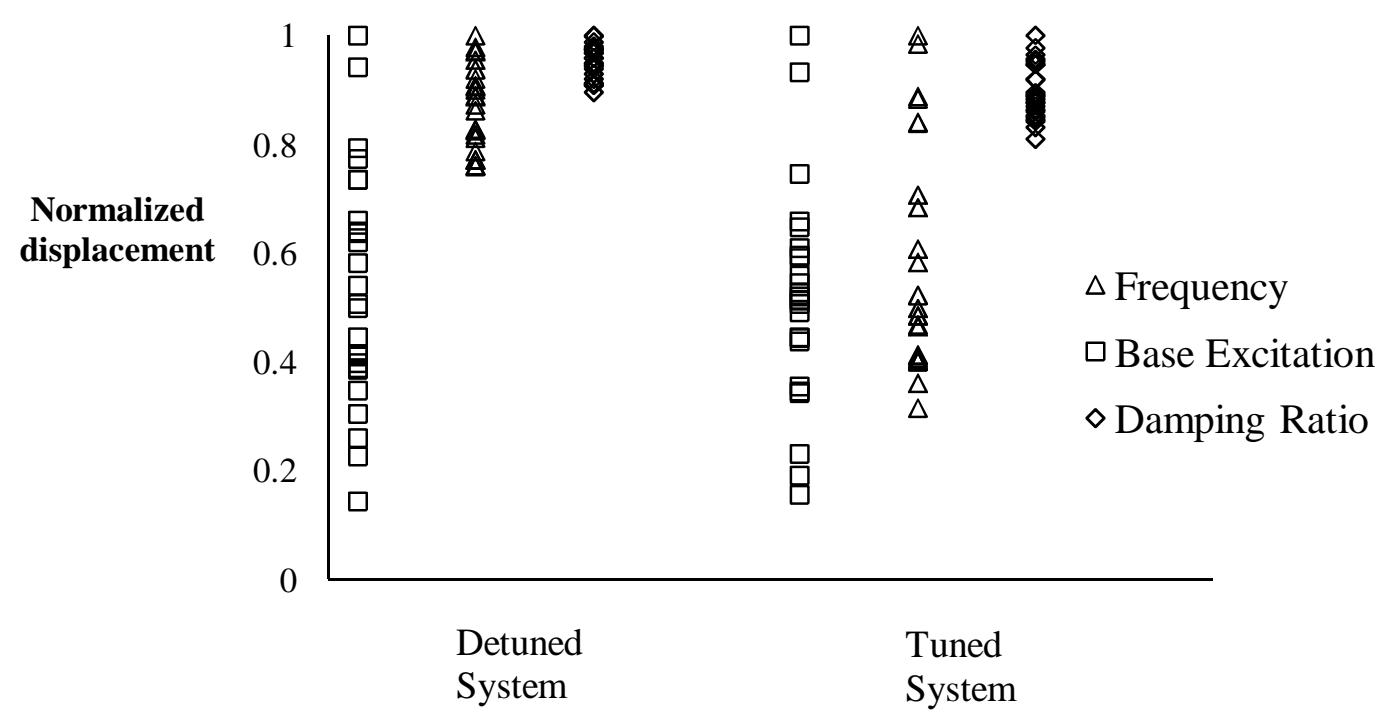

Figure 5.2: Relative significance of uncertainties in earthquake input and modal properties on the secondary system response.

As evident from Figure 5.2, the effect of uncertainty in base excitation has relatively greater significance in the case of detuned systems. However in tuned systems, the effect of uncertainty in uncoupled frequencies is similar to that due to the uncertainty in base excitation. Hence, possibility of tuning between various modes of primary and secondary systems tends to affect the response of secondary systems appreciably. It can also be seen that in the tuned systems, the uncertainties in base excitation and frequencies have relatively greater influence compared to that of damping ratios alone. 


\subsection{Examples}

As seen in Section 5.1, uncertainties in uncoupled frequencies and modal damping ratios can play a vital role in influencing the secondary system response of a tuned system. Hence, it is necessary to consider variations in modal properties of tuned systems along with uncertainties in base excitation in order to calculate the design response of the secondary system. Chapter 4 introduced the SRMS method of determining the design response as proposed by Gupta and Choi (2005). In this section, the application and verification of the SRMS method of determining the secondary system design response for multi-degree of freedom systems is illustrated using two different coupled system configurations. Different variations of these configurations are considered to evaluate the effect of tuning between modes of primary and secondary systems on the response of the secondary system. For each coupled system, the evaluation of the design response using SRMS method is illustrated using the following steps:

1. To model the uncertainties associated with modal properties of uncoupled systems, the natural frequencies and damping ratios are assumed to be uniform random variables varying within $\pm 15 \%$ of their respective mean values. 100 sets of randomly sampled uncoupled frequencies and modal damping ratios for primary and secondary systems are generated for each coupled system using the formulation developed in Chapter -3. 
2. The variation in input excitation is considered through 75 real earthquake records all normalized to a unit PGA. The details of the earthquake records are given in Table B.1 of Appendix - B. For estimation of design responses using response spectrum method, the design spectrum is specified at a non-exceedence probability of 0.84 over the 75 earthquakes (see Appendix - B). This design spectrum forms the input at the base of the primary system for the response spectrum analyses.

3. Using the design spectrum developed in Step 2, the conditional responses of the secondary system are obtained from multiple response spectrum analyses. The 100 responses obtained from response spectrum analysis using each of the 100 sets of randomly generated modal properties are used to determine the SRMS value of the design response, $R_{0.84}$ using Equation (4.27).

4. To validate and compare the design responses from the SRMS method, the design response value is directly obtained from conducting multiple time history analyses. A total of 7500 responses are evaluated by considering combinations of 75 earthquakes and 100 sets of randomly sampled frequencies and modal damping ratios. The secondary system design response $R_{0.84}$ is statistically determined by Equation (4.1) over these 7500 responses.

5. Finally, the values of SRMS responses from Step 3 are compared with the design responses evaluated from multiple time history analyses in Step 4 for each system. 
Example-A: 2-DOF primary-2-DOF secondary coupled system (singly-connected)

In this example, five different variations (A-1 to A-5) of the model shown in Figure 5.1 are considered to illustrate the application of SRMS method to singly-connected MDOF primary - MDOF secondary systems. The models are designed as described below in order to study the effect of different kinds of tuning between primary and secondary system modes:

- Model A-1 considers a case where the fundamental mode of the primary system is tuned with the fundamental mode of the secondary system.

- Model A-2 is the case where the fundamental mode of the primary system is tuned with the second mode of the secondary system.

- Model A-3 is the case where the first mode of the primary system is tuned with the first mode of the secondary system and the second mode of the primary system is tuned with the second mode of the secondary system.

- Model A-4 is the case where the fundamental mode of the primary system is tuned with the first as well as second mode of the secondary system. The two modes of the secondary system are closely-spaced.

- Model A-5 considers a case where the fundamental mode of the secondary system can be tuned with either of the modes of the primary system. Both the modes of the primary system are closely-spaced. 
The details of models are presented in Tables 5.2 and 5.3. The mean values of modal damping ratios for the uncoupled primary system and secondary system are considered to be $6 \%$ and $2 \%$ respectively.

In models A-1 to A-3, the uncoupled natural frequencies of both primary and secondary systems are far apart. Therefore, the frequencies can be treated as independent random variables for generating the random sample sets required in Step 1. However, the secondary system modes of model A-4 $(3.81 \mathrm{~Hz} \& 4.21 \mathrm{~Hz})$ and primary system modes of model A-5 $(3.81 \mathrm{~Hz} \& 4.21 \mathrm{~Hz})$ are closely-spaced with overlapping probability density functions. This means that the frequencies cannot be sampled independently. To avoid incorrect sample sets, a joint probability density function between the closely-spaced frequencies based on the formulation developed in Chapter - 3 has been used for generating the random sample sets. Figure 5.3 plots the probability density functions of the sampled frequencies of the secondary system of model A-4. 
Table 5.2: Properties of uncoupled primary and secondary systems in Example - A

\begin{tabular}{|c|c|c|c|c|c|c|c|c|}
\hline \multirow{2}{*}{$\begin{array}{c}\text { Model } \\
\#\end{array}$} & \multicolumn{2}{|c|}{$\begin{array}{c}\text { Mass - Primary } \\
\text { System }(\mathrm{Kg})\end{array}$} & \multicolumn{2}{|c|}{$\begin{array}{c}\text { Stiffness - Primary } \\
\text { System }\left(\mathrm{Nm}^{-1}\right)\end{array}$} & \multicolumn{2}{c|}{$\begin{array}{c}\text { Mass - } \\
\text { Secondary } \\
\text { System }(\mathrm{Kg})\end{array}$} & \multicolumn{2}{c|}{$\begin{array}{c}\text { Stiffness - Secondary } \\
\text { System }\left(\mathrm{Nm}^{-1}\right)\end{array}$} \\
\cline { 2 - 9 } & $m_{p 1}$ & $m_{p 2}$ & $k_{p 1}$ & $k_{p 2}$ & $m_{s 1}$ & $m_{s 2}$ & $k_{s 1}$ & $k_{s 2}$ \\
\hline A-1 & $3 \times 10^{5}$ & $2 \times 10^{5}$ & $5 \times 10^{8}$ & $5 \times 10^{8}$ & 500 & 300 & $7.97 \times 10^{5}$ & $5.80 \times 10^{5}$ \\
\hline A-2 & $3 \times 10^{5}$ & $2 \times 10^{5}$ & $7.5 \times 10^{7}$ & $7.5 \times 10^{7}$ & 500 & 300 & $7.7 \times 10^{5}$ & $5.6 \times 10^{5}$ \\
\hline A-3 & $3 \times 10^{5}$ & $2 \times 10^{5}$ & $5 \times 10^{8}$ & $5 \times 10^{8}$ & 500 & 300 & $7.98 \times 10^{5}$ & $7.98 \times 10^{5}$ \\
\hline A-4 & $5 \times 10^{5}$ & $1 \times 10^{5}$ & $3.91 \times 10^{8}$ & $3.55 \times 10^{8}$ & 3000 & 30 & $1.9 \times 10^{6}$ & $1.9 \times 10^{4}$ \\
\hline A-5 & $3 \times 10^{6}$ & $3 \times 10^{4}$ & $1.9 \times 10^{9}$ & $1.9 \times 10^{7}$ & 500 & 100 & $3.9 \times 10^{5}$ & $3.5 \times 10^{5}$ \\
\hline
\end{tabular}

Table 5.3: Mean values of natural frequencies for uncoupled primary and secondary systems for Example- A

\begin{tabular}{|c|c|c|c|c|c|}
\hline \multirow{2}{*}{$\begin{array}{c}\text { Model } \\
\#\end{array}$} & \multirow{2}{*}{$\begin{array}{c}\text { Model } \\
\text { Description }\end{array}$} & \multicolumn{2}{|c|}{$\begin{array}{c}\text { Natural Frequencies } \\
\text { Primary System }(\mathrm{Hz})\end{array}$} & \multicolumn{2}{|c|}{$\begin{array}{c}\text { Natural Frequencies - } \\
\text { Secondary System (Hz) }\end{array}$} \\
\cline { 3 - 6 } & $\omega_{p 1}$ & $\omega_{p 2}$ & $\omega_{s 1}$ & $\omega_{s 2}$ \\
\hline $\mathrm{A}-1$ & $\omega_{p 1} \approx \omega_{s 1}$ & 4.6 & 11.25 & 4.5 & 9.9 \\
\hline $\mathrm{A}-2$ & $\omega_{p 1} \approx \omega_{s 2}$ & 1.78 & 4.36 & 4.4 & 9.76 \\
\hline $\mathrm{A}-3$ & $\begin{array}{c}\omega_{p 1} \approx \omega_{s 1} \\
\omega_{p 2} \approx \omega_{s 2}\end{array}$ & 4.59 & 11.25 & 4.63 & 11.25 \\
\hline $\mathrm{A}-4$ & $\begin{array}{c}\omega_{p 1} \approx \omega_{s 1} \\
\omega_{p 1} \approx \omega_{s 2}\end{array}$ & 3.99 & 10.58 & 3.81 & 4.21 \\
\hline $\mathrm{A}-5$ & $\begin{array}{c}\omega_{p 1} \approx \omega_{s 1} \\
\omega_{p 2} \approx \omega_{s 1}\end{array}$ & 3.81 & 4.21 & 3.99 & 10.58 \\
\hline
\end{tabular}




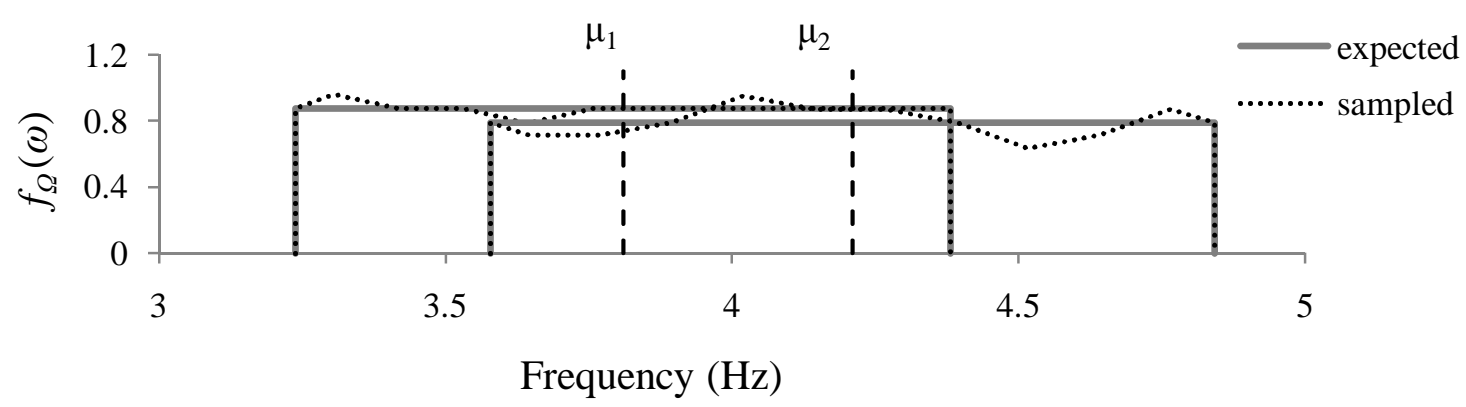

Figure 5.3: Probability density function of sampled values of frequencies of the secondary system in model A-4

After randomly generating the sample sets of modal properties, the secondary system design response values of each model are obtained by conducting multiple time history and response spectrum analyses as described in Steps 3 and 4. Figures $5.4-5.8$ compare the secondary system design response from the time history analyses and the SRMS value evaluated from multiple response spectrum analyses. The figures also plot the value of response corresponding to a non-exceedence probability of 0.84 over the responses from the multiple response spectrum analyses (Step 3). 


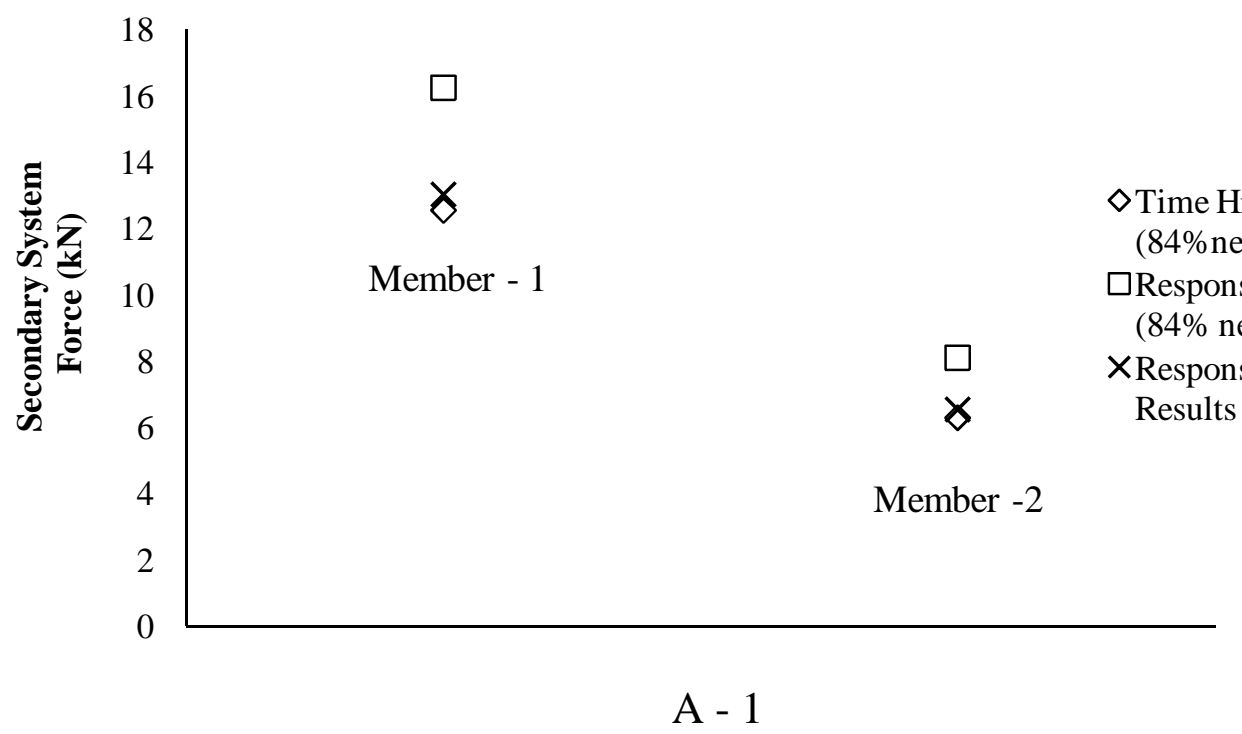

Figure 5.4: Secondary system design responses for model A-1 (Example - A)

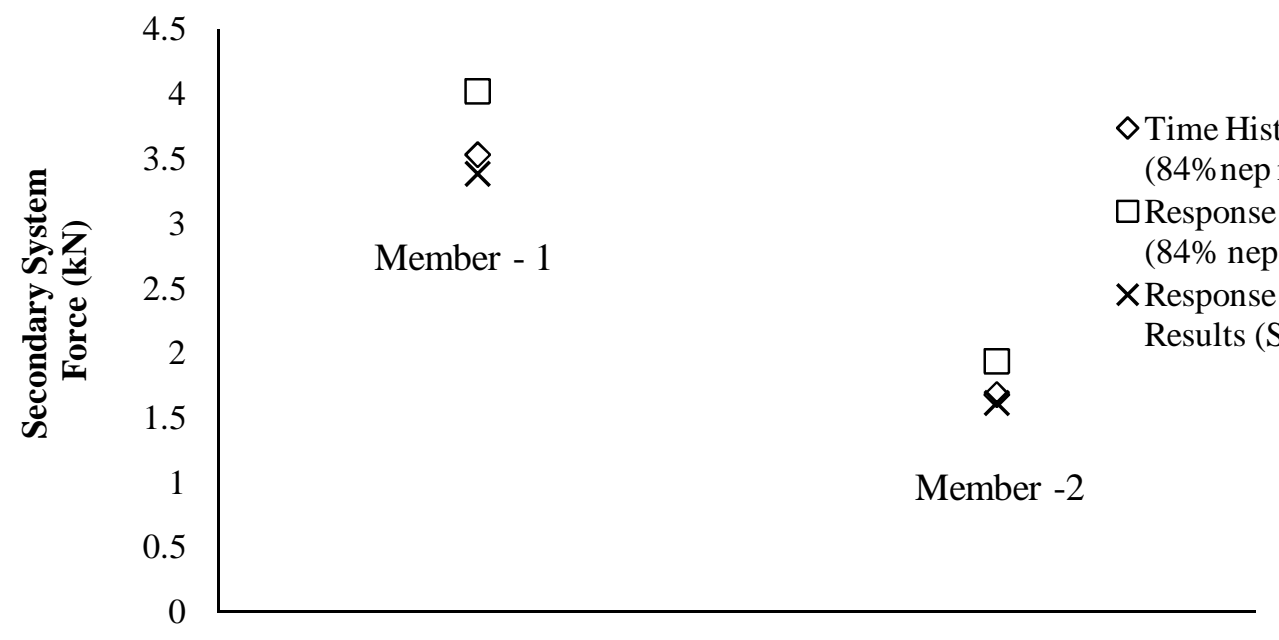

A - 2

Figure 5.5: Secondary system design responses for model A-2 (Example - A) 


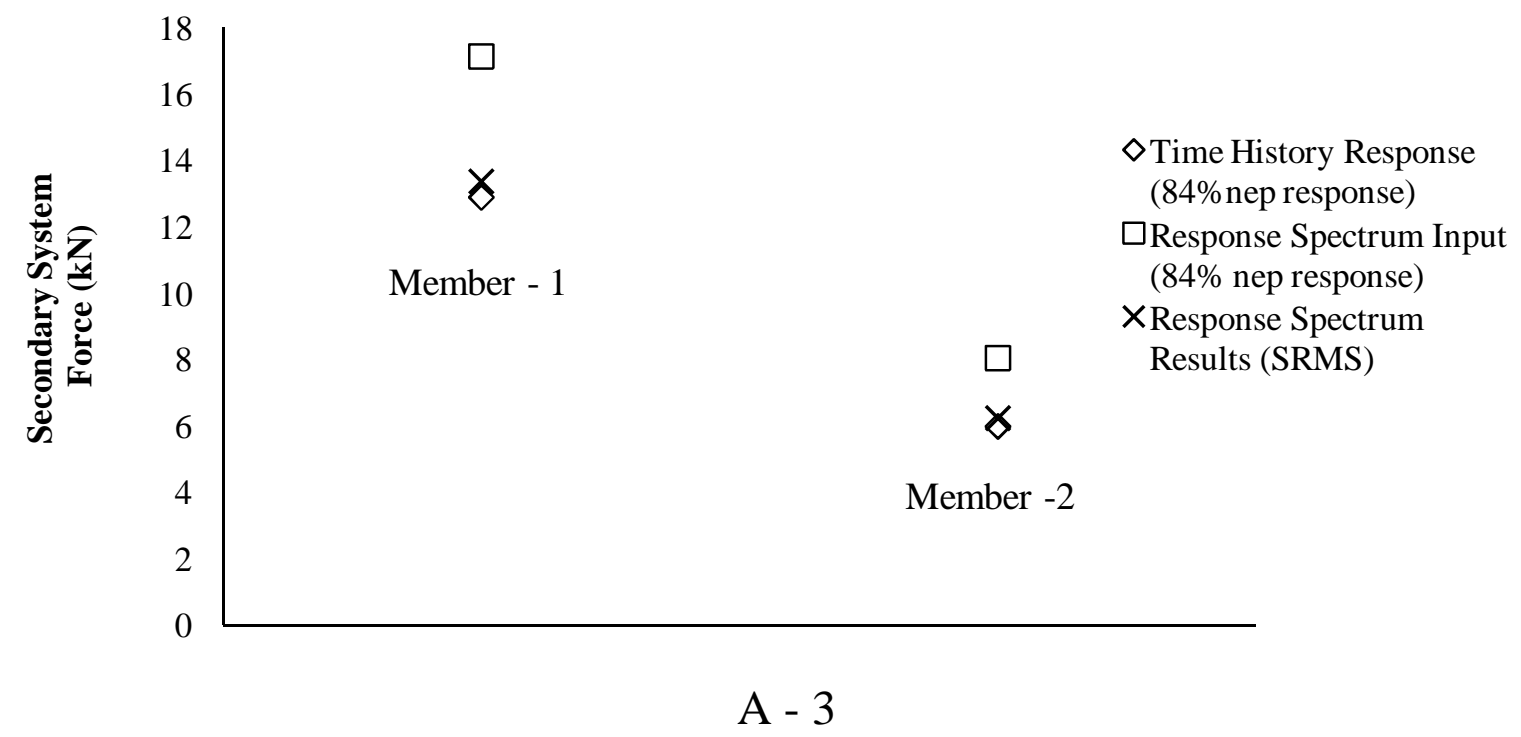

Figure 5.6: Secondary system design responses for model A-3 (Example - A)

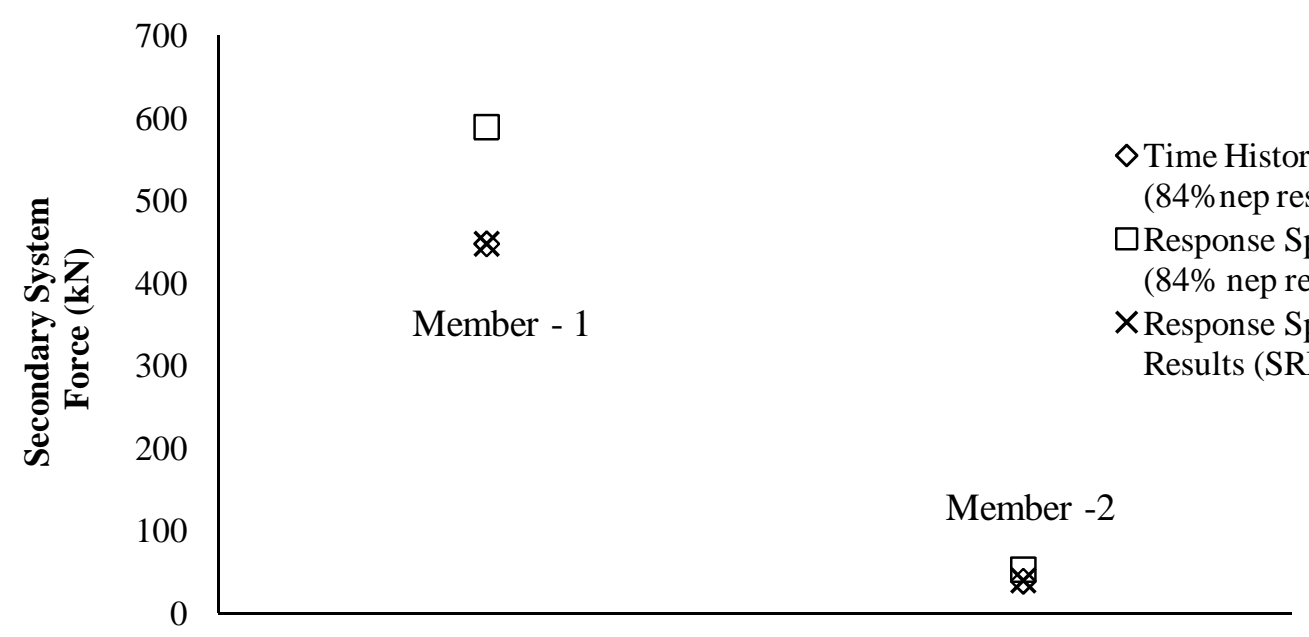

\section{A - 4}

Figure 5.7: Secondary system design responses for model A-4 (Example - A) 


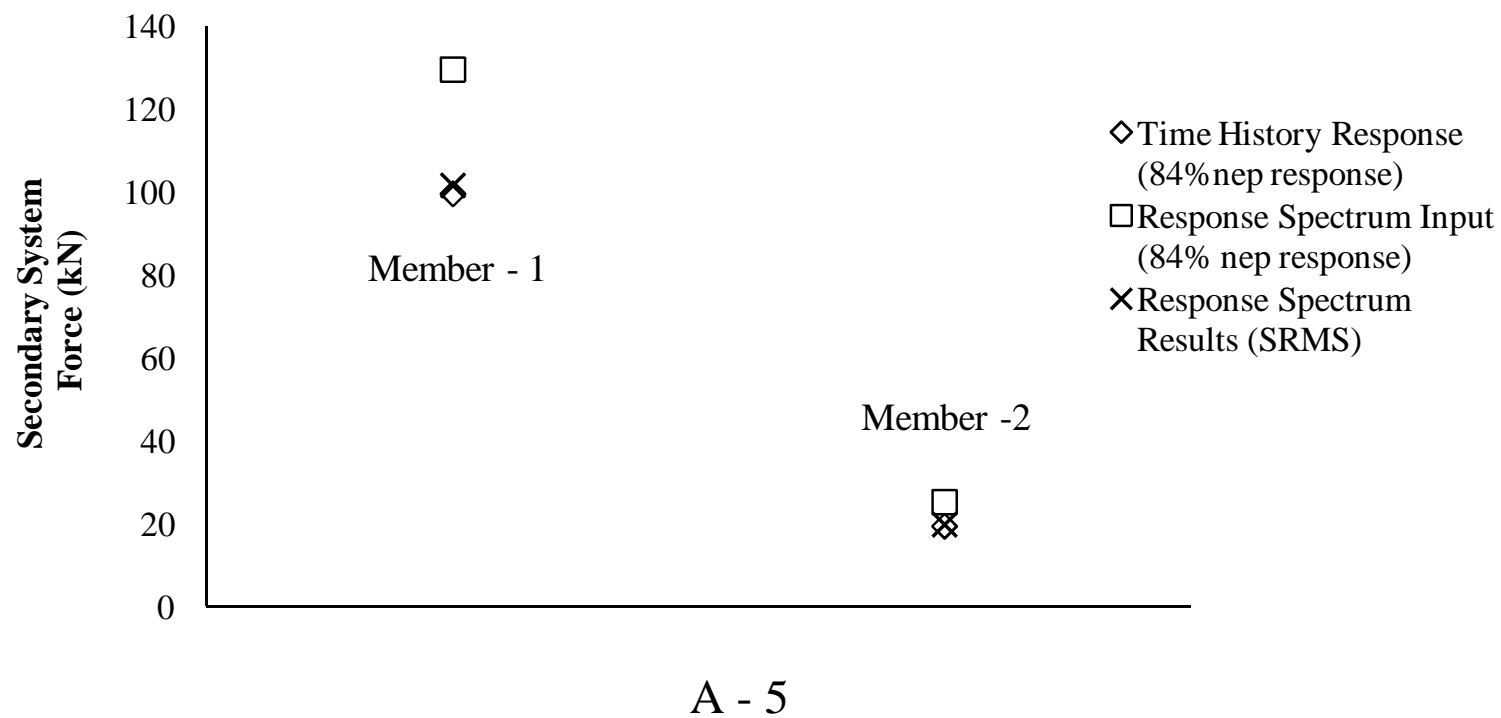

Figure 5.8: Secondary system design responses for model A-5 (Example - A)

Table 5.4 presents the error between the design responses from the SRMS method and the design response from multiple time history analyses. It can be seen from Figures 5.4 -5.8 that the design value of element forces for all the five models obtained from the SRMS method agree very well with the design value of forces obtained from the multiple time history analyses. However, the design response specified at a non-exceedence probability of 0.84 over multiple response spectrum analyses is excessively conservative with respect to the corresponding design response evaluated from time history analyses in all cases. 
Table 5.4: Comparison between the values of design member forces in the secondary system obtained from time history input and SRMS method (Example - A)

\begin{tabular}{|c|c|c|c|c|}
\hline \multirow{2}{*}{$\#$} & \multirow{2}{*}{ MODEL } & \multicolumn{2}{|c|}{$R_{\mathbf{0 . 8 4}}(\mathbf{k N})$} & \multirow{2}{*}{ Error (\%) } \\
\cline { 3 - 5 } & & $\begin{array}{c}\text { Time history input } \\
\text { (Equation 4.1) }\end{array}$ & $\begin{array}{c}\text { SRMS Method } \\
\text { (Equation 4.27) }\end{array}$ & \\
\hline \multirow{2}{*}{ A-1 } & 1 & 12.55 & 13.04 & $3.9 \%$ \\
\cline { 2 - 5 } & 2 & 6.29 & 6.54 & $4.0 \%$ \\
\hline \multirow{2}{*}{ A-2 } & 1 & 3.53 & 3.38 & $-4.3 \%$ \\
\cline { 2 - 5 } & 2 & 1.68 & 1.61 & $-4.1 \%$ \\
\hline \multirow{2}{*}{ A-3 } & 1 & 12.88 & 13.36 & $3.7 \%$ \\
\cline { 2 - 5 } & 2 & 6.00 & 6.23 & $3.8 \%$ \\
\hline \multirow{2}{*}{ A-4 } & 1 & 447.19 & 447.78 & $0.1 \%$ \\
\cline { 2 - 5 } & 2 & 38.65 & 39.14 & $1.3 \%$ \\
\hline \multirow{2}{*}{ A-5 } & 1 & 99.69 & 101.64 & $2.0 \%$ \\
\cline { 2 - 5 } & 2 & 19.45 & 19.87 & $2.2 \%$ \\
\hline
\end{tabular}

Example - B: Multiply-Connected 8-DOF primary - 3-DOF secondary coupled system

In Example - B, an 11-DOF coupled system model as shown in Figure 5.9 is considered to illustrate the applicability of the SRMS method to coupled systems in which the secondary system is connected at multiple locations to the primary system. The case of multiple modes of primary system being tuned with a secondary system mode is also considered in this example. The primary system consists of eight storeys with massless shear beam elements and lumped masses and is fixed at the ground. The $i^{\text {th }}$ storey masses and $i^{\text {th }}$ storey stiffness of the primary system are denoted by $m_{p i}$ and $k_{p i}$ respectively. The secondary 
system comprises of four shear beam elements (stiffness $\left.k_{s j}\right)$ and three lumped masses $\left(m_{s j}\right)$. The secondary system is connected to the primary system at two different loactions as shown. Two different variations of the model are considered as follows:

- Case B-1 considers a case where the fundamental mode of the primary system is tuned with the fundamental mode of the secondary system.

- Case B-2 examines a case where a secondary system mode can be tuned with either of the two closely-spaced modes of the primary system. Here, the fundamental mode of the secondary system can be tuned with either of the third or the fourth mode of the primary system.

The properties of models B-1 and B-2 are given in Figures 5.9 and 5.10 respectively. Table 5.5 lists the uncoupled frequencies and modal damping ratios of primary and secondary systems of models B-1 and B-2. 


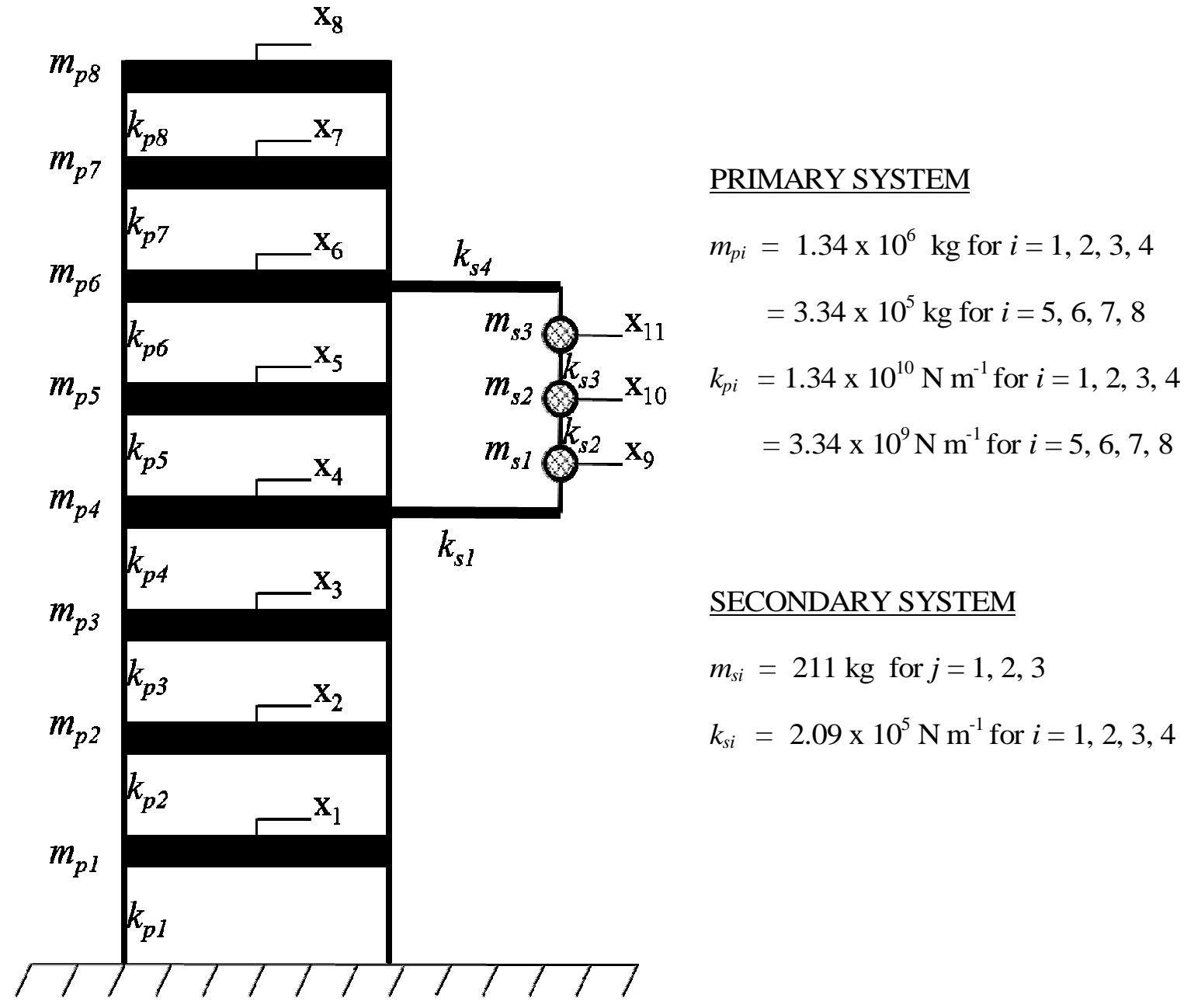

Figure 5.9: The 8 DOF primary - 3 DOF secondary system structure for model B-1 


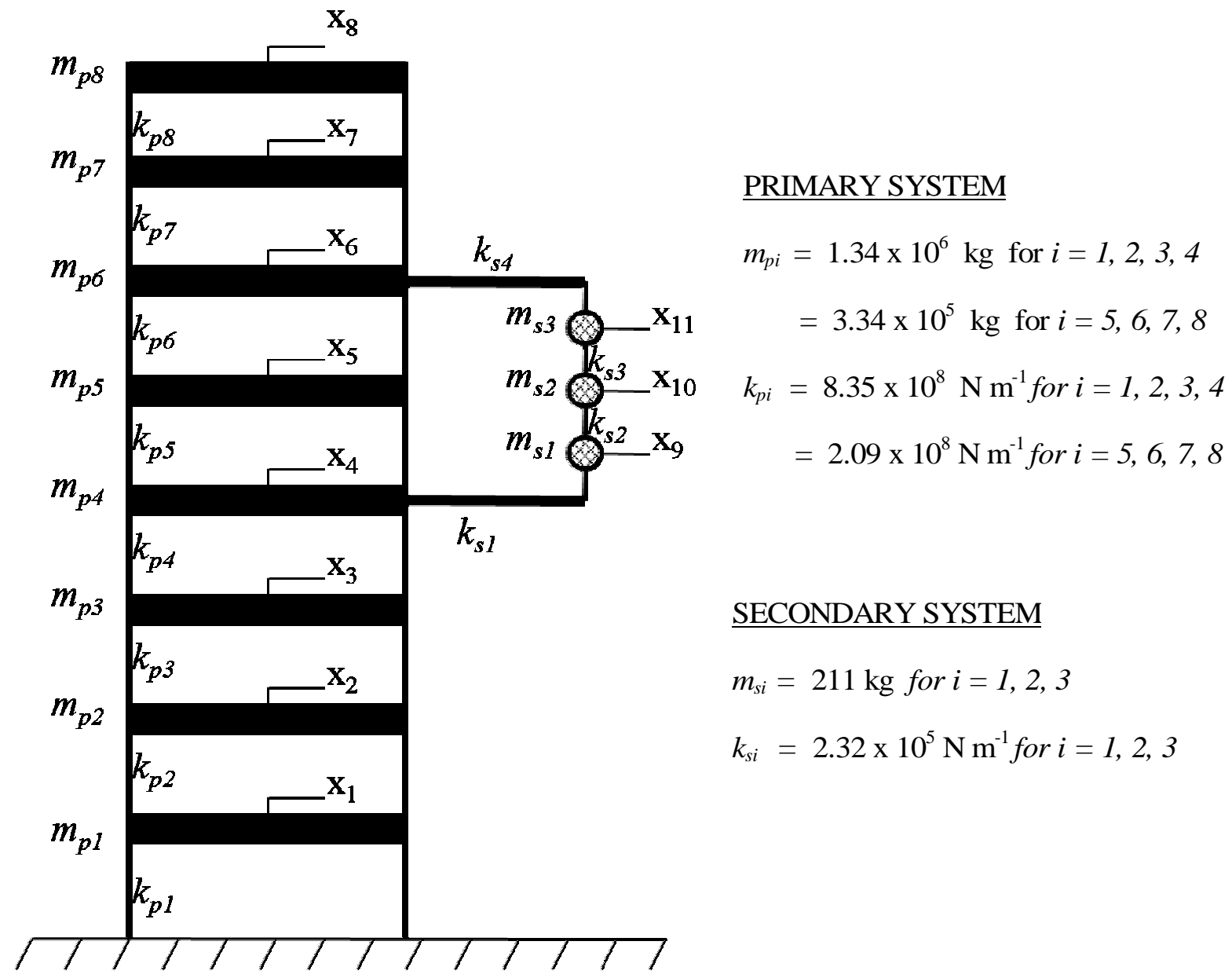

Figure 5.10: The 8 DOF primary -3 DOF secondary structure for model B-2 
Table 5.5: Modal properties of uncoupled primary and secondary systems in Example - B

\begin{tabular}{|c|c|c|c|c|c|c|}
\hline \multirow{2}{*}{$\begin{array}{c}\text { Model } \\
\quad \#\end{array}$} & \multirow{2}{*}{$\begin{array}{c}\text { Model } \\
\text { Description }\end{array}$} & \multicolumn{4}{|c|}{ UNCOUPLED FREQUENCIES (Hz) } & \multirow{2}{*}{$\begin{array}{c}\text { DAMPING } \\
\text { RATIO }\end{array}$} \\
\hline & & \multicolumn{2}{|c|}{ Primary System } & \multicolumn{2}{|c|}{ Secondary System } & \\
\hline \multirow{8}{*}{ B-1 } & \multirow{8}{*}{$\omega_{p 1} \approx \omega_{s l}$} & $\omega_{p 1}$ & 4.0 & $\omega_{s l}$ & 3.8 & \multirow{8}{*}{$\begin{array}{l}\xi_{p}=0.06 \\
\xi_{s}=0.02\end{array}$} \\
\hline & & $\omega_{p 2}$ & 7.3 & $\omega_{s 2}$ & 7.1 & \\
\hline & & $\omega_{p 3}$ & 14.8 & $\omega_{s 3}$ & 9.3 & \\
\hline & & $\omega_{p 4}$ & 17.5 & & & \\
\hline & & $\omega_{p 5}$ & 23.8 & & & \\
\hline & & $\omega_{p 6}$ & 25.4 & & & \\
\hline & & $\omega_{p 7}$ & 29.8 & & & \\
\hline & & $\omega_{p 8}$ & 30.2 & & & \\
\hline \multirow{8}{*}{ B-2 } & \multirow{8}{*}{$\begin{array}{l}\omega_{p 3} \approx \omega_{s 1} \\
\omega_{p 4} \approx \omega_{s 1}\end{array}$} & $\omega_{p 1}$ & 1.0 & $\omega_{s l}$ & 4.0 & \multirow{8}{*}{$\begin{array}{l}\xi_{p}=0.06 \\
\xi_{s}=0.02\end{array}$} \\
\hline & & $\omega_{p 2}$ & 1.8 & $\omega_{s 2}$ & 7.5 & \\
\hline & & $\omega_{p 3}$ & 3.7 & $\omega_{s 3}$ & 9.8 & \\
\hline & & $\omega_{p 4}$ & 4.4 & & & \\
\hline & & $\omega_{p 5}$ & 6.0 & & & \\
\hline & & $\omega_{p 6}$ & 6.4 & & & \\
\hline & & $\omega_{p 7}$ & 7.4 & & & \\
\hline & & $\omega_{p 8}$ & 7.6 & & & \\
\hline
\end{tabular}

It can be seen that in primary systems of both models, the frequency sets $\left(\omega_{p 3}, \omega_{p 4}\right)$ and $\left(\omega_{p 5}, \omega_{p 6}, \omega_{p 7}, \omega_{p 8}\right)$ are closely-spaced. The individual probability density functions of these frequencies overlap. Hence, the simulation of random sample sets for the uncoupled frequencies cannot be achieved by independent sampling. The sampling for each model is conducted based on the formulation developed in Chapter- 3. Figure 5.11 compares the 
probability density functions of sampled frequencies with the expected uniform distributions for primary system frequencies in Model B-2.

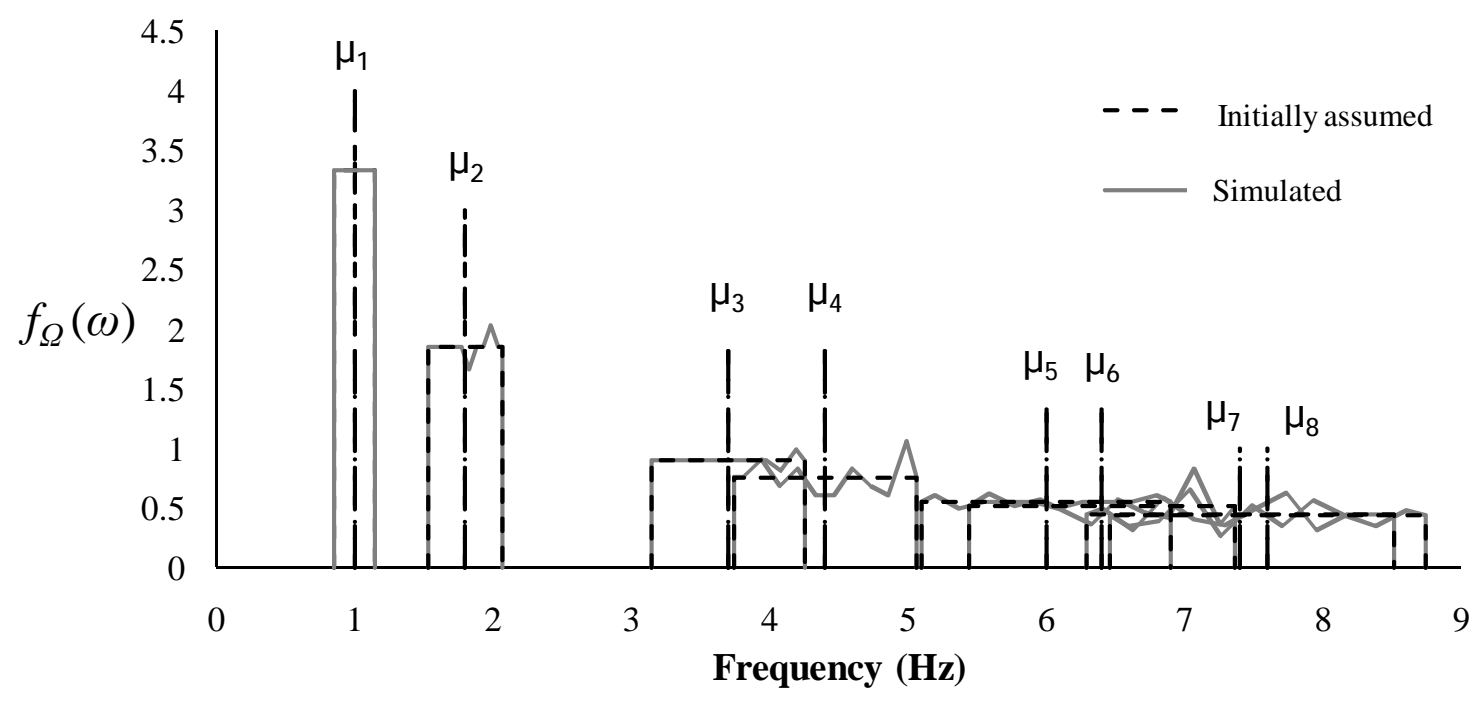

Figure 5.11: Probability density functions of sampled uncoupled frequencies of primary system in Model B-2

Following the similar procedure as in Example - A, the design values of forces in the secondary system are computed from SRMS method as well as from Monte Carlo simulations with time history input. The design secondary system response values $R_{0.84}$ from both methods are compared in Figures 5.12 for model B-1 and Figure 5.13 for B-2. The SRMS values of design responses are close to the design responses obtained from Monte Carlo simulation with time history input. Also, the design response value specified at $84 \%$ 
NEP over all responses from multiple response spectrum analyses yielded excessively conservative responses.

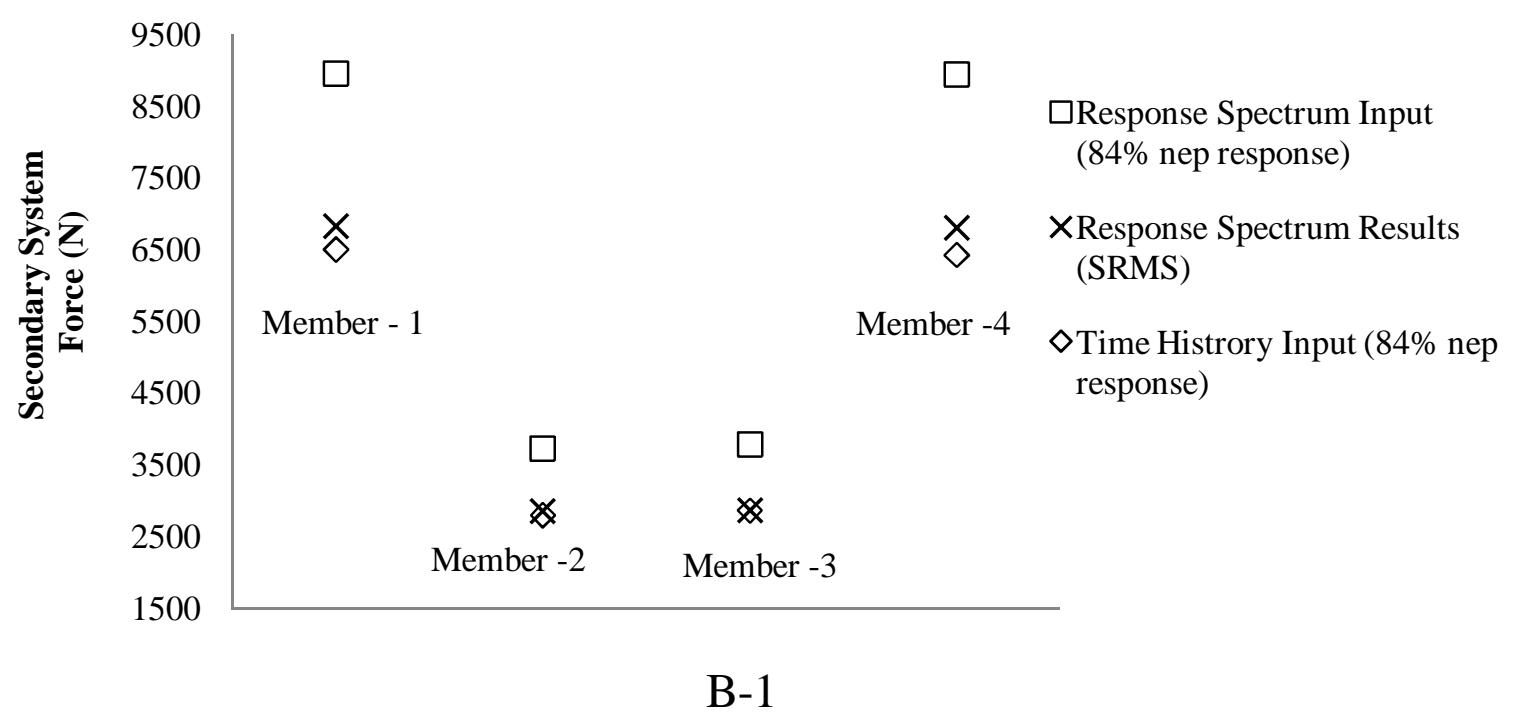

Figure 5.12: Secondary system design responses for model B-1 (Example - B) 


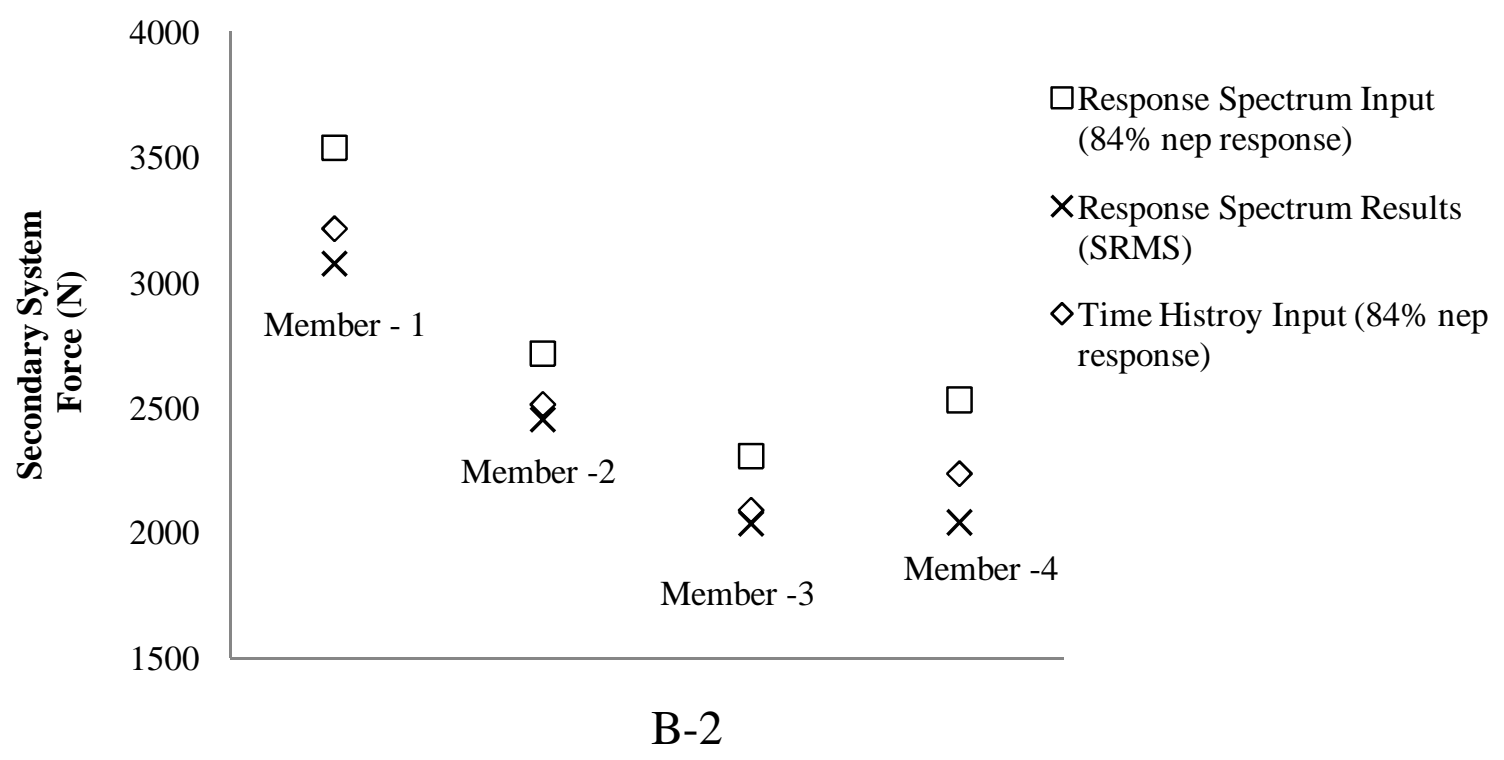

Figure 5.13: Secondary system design responses for model B-2 (Example - B) 


\section{CHAPTER - 6 \\ SUMMARY, CONCLUSIONS AND FUTURE WORK}

\subsection{Summary}

This study investigates the effect of uncertainties in modal properties of uncoupled MultiDegree-of-Freedom (MDOF) primary - MDOF secondary systems in the seismic analysis of non-classically damped coupled systems. Gupta and Choi (2005) proposed the Square-rootof-mean-of-squares (SRMS) approach to determine the design response corresponding to a non-exceedence probability of 0.84 using multiple response spectrum analyses and illustrated its application to SDOF primary - SDOF secondary systems. This thesis focuses on verification and application of the SRMS approach to MDOF primary - MDOF secondary systems.

As illustrated in Gupta and Choi (2005), it was found that the uncertainties in uncoupled frequencies have a significant effect on the secondary system response in tuned or nearly tuned MDOF coupled systems. On the other hand, uncertainties in modal properties have relatively negligible effect as compared to uncertainties in base excitations on the response of secondary systems in the case of detuned primary - secondary systems. Unlike Gupta and Choi (2005) which considers only SDOF primary - SDOF secondary systems, 
emphasis is placed on studying the behavior of tuned MDOF primary - MDOF secondary systems.

Implementing the SRMS method comprises of conducting multiple analyses of coupled system with randomly sampled sets of uncoupled natural frequencies of primary and secondary systems. Random samples of frequencies require knowledge of their probability density functions. Closed-form solutions for probability density functions of natural frequencies of simply-supported flexural beams considering variations in flexural rigidity were derived. It was seen that the density functions of the frequencies for lower order modes are relatively narrow banded and do not overlap in any meaningful way. However, there is a significant degree of overlap among the density functions for the frequencies of higher modes. The observations were similar for the frequencies of shear beam considering variations in shear rigidity.

A significant overlap in probability density functions of uncoupled frequencies can give incorrect set of frequencies in the sense that the frequencies do not remain as ordered pairs or ordered sets. The frequencies of any uncoupled primary or secondary system should all be in an ascending order. If the uncertainty in the structure is dominated by a single parameter, the natural frequencies can be independently simulated without any errors because the correlation between any two frequencies is unity. However, if uncertainties exist in multiple structural parameters, the correlations between the frequencies are not always unity. 
There are numerous methods to estimate correlations and higher order statistics for natural frequencies of a structure given the probabilistic characteristics of material and geometrical properties of the structure. It is, however, impractical to consider the various uncertainties in all the structural parameters to estimate the correlations between the frequencies of a real-life structure. Even if the correlations among the frequencies of a structure are known, the problem of disordered sampled sets of random natural frequencies is unavoidable unless the frequencies are perfectly correlated. This is usually not the case.

USNRC (1978) recommends a $\pm 15 \%$ uncertainty in the frequencies of the primary system in order to account the effect of uncertainties on secondary system response. It is reasonable to consider a uniform distribution of these frequencies within the prescribed frequency region of interest. Independent sampling of frequencies can result in disordered samples if the modes of a structure are closely-spaced. Moreover, rejecting the incorrect samples result in individual density functions that are significantly different from the distributions initially assumed for sampling of each natural frequency.

One way to overcome the above constraints is to consider them in an implicit manner within the sampling scheme. A formulation for a joint probability density function is derived using fundamental probability approaches. Using the joint density function, it was possible to sample sets of natural frequencies such that they were always ordered sets. Also, each frequency set passed the Kolmogorov-Smirnov test with confidence limits of 0.03 for uniform distribution between $\pm 15 \%$ of its mean value. 
The implementation of the SRMS approach to MDOF primary - MDOF secondary systems was illustrated for two kinds of configurations - singly- and multiply-connected coupled systems. Various degrees of tuning between the primary and secondary systems were considered. The possibility of a single secondary system mode to be tuned with either of the two closely-spaced primary system modes was also considered. 100 randomly sampled sets of uncoupled frequencies and modal damping ratios for primary and secondary systems were generated for each model. The uncertainty in base excitation was accounted by considering 75 real earthquake records normalized to the same value of peak ground acceleration (PGA). The input spectrum corresponding to a non-exceedence probability of 0.84 for multiple response spectrum analyses was generated from the earthquake time histories. The SRMS value of the design response of the secondary systems was compared with the design response obtained directly as the response value corresponding to a nonexceedence probability of 0.84 from multiple time history analyses performed by considering combinations of the 75 earthquakes and 100 modal property sets. The design response values calculated using SRMS method agreed well with the design responses calculated from multiple time history analyses.

\subsection{Conclusions}

The specific conclusions related to the various tasks outlined in the Section 1.4 of this thesis are: 
- The closed-form expressions of the probability density functions of the natural frequencies of a simply supported beam derived by considering variations in flexural rigidity indicate that the probability density functions of the higher order modes have significant overlap. Similar observations can be found for a simply-supported beam with variations in shear rigidity.

- If the uncertainty in a structure is dominated by a single parameter, the correlation coefficient between any two frequencies is unity indicating a linear relationship between any two frequencies in the structure. However, if the uncertainty exists in multiple parameters in a structure, the correlation coefficient between the frequencies is much less than unity.

- If the probability density functions of frequencies with closely-spaced modes have significant overlap, the independently sampled sets of natural frequencies may not be in correct order. Rejection of incorrect sample sets result in probability distribution functions of the frequencies that are significantly different from their assumed distributions.

- A formulation for joint probability density function for uncoupled natural frequencies is developed from fundamental probability approaches. Sampling the natural frequencies according to the formulation ensures that the random sample sets of natural frequencies are always in order. Also, the individual density functions of the 
sampled frequencies satisfy the Kolmogorov-Smirnov test with confidence limits of 0.03 for the assumed distributions.

- The application of the SRMS method is illustrated through numerical examples. The random sample sets of uncoupled natural frequencies of MDOF primary and secondary systems were generated from the formulation developed.

- The SRMS method is illustrated for the following numerical examples:

B Five different variations studying the effect of different tuning characteristics between the primary and secondary system modes of a singly-connected 2 DOF primary - 2 DOF secondary systems are considered.

B 2 different variations of an 8 DOF primary - 3 DOF secondary systems are considered. The 8 DOF primary systems have closely-spaced modes. One of the variations studies the effect of a single secondary system mode being tuned with multiple primary system modes.

The design response determined from the SRMS method using response spectrum analyses is in good agreement with the design response determined from multiple time history analyses for all the systems.

\subsection{Limitations and Recommendations for Future Work}

The formulation developed for the joint density function for sampling random sets of natural frequencies of a structure satisfactorily ensures that the sampled frequencies are ordered sets 
and maintain the individual density functions for each frequency. The formulation of the density function assumes the property of central symmetry for the distribution. Such an assumption leads to high correlations for modes with closely-spaced frequencies. If any two consecutive frequencies are more than $35.3 \%$ apart, the correlation is considered to be minimal, and the correlation coefficient between the frequencies increases along with increasing closeness between the means of the frequencies. In short, the joint density function implicitly assumes that if there is any change in the structural properties that caused a shift in a frequency, the same change is likely to cause a proportional shift in a frequency that is closely spaced to the former. While this assumption is reasonable and could hold well in most instances, it may not always be the case i.e. there could be high correlations between modes with frequencies far apart as well. However, generalization of such behavior is difficult. Future work can focus on better understanding of the correlations between uncoupled natural frequencies of a structure and their various statistics to be implemented into the sampling scheme.

The thesis assumes that the uncoupled frequencies and modal damping ratios are random variables of uniform distribution within $\pm 15 \%$ of their respective mean values. The effect of modeling the modal properties using other probability distributions on the response of secondary system and applicability of SRMS method could be studied.

Application of the proposed SRMS approach has not been illustrated for any real-life coupled systems. The applicability of this method may be studied for such systems. 


\section{REFERENCES}

A.Gupta, B.Choi, "Consideration of uncertainties in seismic analysis of coupled building piping systems," Nuclear Engrg. and Des., 235, 2071-2086, 2005.

A.K.Gupta and J.W.Jaw, "Seismic Response of non-classically damped systems", Nuclear Engineering and Design Vol 91, 153-159, January 1986.

A.K.Gupta, Response Spectrum Method in Seismic Analysis and Design of Structures, CRC Press, Inc., Boca Raton, FL, 1992.

T.Igusa and A Der Kiureghian, "Interaction in Primary-Secondary Systems," J. Pressure Vessel Technology, 114, 53-59, 1992.

A.Gupta and A.K.Gupta, "Missing mass effect in coupled analysis, I: complex modal properties," J. struct. Engrg., ASCE, 124(5), 490-495, 1998.

A.Gupta and A.K.Gupta, "Application of new developments in coupled seismic analysis of piping systems," Transactions of the 13th Internationsal conference of Structural Mechanics in Reactor Technology, Porto Alegre, Brazil, August, 1995.

A.Gupta and P.Aradhya, "Snubber Reduction Using CREST program." Current Issues Related to Nuclear Power Plant Structures, Equipment and Piping, Proceedings of the 6th International Symposium, Raliegh, North Carolina, December, 1996.

A.K.Gupta, T.Hassan and A.Gupta, "Correlation coefficients for modal response combination of non-classically damped systems," Nuclear Engrg. and Des., 165, 67-80, 1996. 
Y.K.Lin, Probabilistic Theory of Structural Dynamics, McGraw-Hill, 1967.

T.Igusa and A Der Kiureghian, "Response of Uncertain Systems to Stochastic Excitation," J. Engrg. Mech. Div., ASCE, 114, 812-832,1988.

H.Jensen and W.D.Iwan, "Response of systems with Uncertain Parameters to Stochastic Excitation," J. Engrg. Mech. Div., ASCE, 118, 1012-1025, 1992.

C.D. Huang, W.Q.Zhu, and T.T.Soong, "Nonlinear Stochastic Response and Reliability of Secondary Systems," J. Engrg. Mech. Div., ASCE, 120, 177-192, 1994.

D.Key, "The influence of damping and parameter uncertainty on seismic secondary response spectra : a random vibration approach," Nuclear Engrg. and Des., 166, 1-7, 1996.

L.K.Liu, C.L.Child and B.Nowotny, "Effect of parameter variation on floor spectra," Speciality Conference on Structural Design of Nuclear Power Plant Facilities, Chicago, 1973.

M.P. Singh, "Seismic Response of Structures with Random Parameters," Proceedings of 7th World Conference on Earthquake Engineering, Istanbul, Turkey, September 1980.

T.Igusa and A Der Kiureghian, "Stochastic Response of systems with Uncertain Properties," Transactions of the 8th International conference of Structural Mechanics in Reactor Technology, Paper M14/5, Brussels, Belgium, August, 1985.

Chen Y.Q., "Modification of floor response spectrum based on stochastic sensitivity analysis," Engineering Structures, Volume 15, Issue 1, Pages 40-46, January 1993. 
J.W. Reed, R.P.Kennedy, B.Lashkari, L.Manuel, "In-structure response for calculating equipment capacities in SMA and SPRA reviews," Current Issues Related to Nuclear Power Plant Structures,

Equipment and Piping, Proceedings of the 5th International Symposium, Lake Buena Vista, Florida, 1994.

U.S. Nuclear Regulatory Commission, Regulatory Guide, Revision 1.122 February, 1978.

American Society of Mechanical Engineers, " ASME boiler and pressure vessel code," ANSI/ASME BPV-III-I-A Section III, Rules of Construction of Nuclear Power Plant Componenets, Division 1, Appendix N, 2007

P. Aradhya, A.Gupta., "Effect of uncertainty in primary system properties on coupled secondary system seismic response", Proceedings of 13th Engineering Mechanics Conference, ASCE, Baltimore, MD, June 13-16, 1999

J.L.Humar, Dynamics of Structures, 2nd Ed., Swets \& Zeitlinger B.V., Lisse, 2002

F.Kozin and J.M. Klosner, "Review of probabilistic analysis of dynamic response of systems with random parameters," NASA Langley Research Center, 1989

Y.K.Lin and G.Q.Cai, Probabilistic Structural Dynamics: Advanced Theory and Applicationss, McGraw-Hill Professional, 2004.

M. Shinozuka and C.J. Astill, "Random eigenvalue problems in structural analysis,". AIAA J. 104 , pp. 456-462, 1972 
T.T.Soong and J.L.Bogandoff, "On the Natural Frequencies of a disordered Linear Chain of N Degrees of Freedom," International Journal of Mechanical Sciences, Vol.5, No.3, 237-265, 1963.

J.D.Collins and W.T.Thomson, "The eigenvalue problem for structural systems with statistical properties," AIAA J 7(4): 642-648, 1969.

T.K.Hasselman and G.C.Hart, "Modal analysis of random structural systems," J. Engrg. Mech. Div., ASCE, 98(EM3), 561-579, 1972.

G.C. Hart, "Eigenvalue uncertainties in stressed structures," J. Engrg. Mech. Div., ASCE, 99(EM3), 481-494, 1973.

D.Song, S.Chen, Z.Qiu,"Stochastic sensitivity anlaysis of eigenvalues and eigenvectors," Comput. Struct. 54(5), 891-896, 1995.

S.Adhikari and M.I.Friswell, "Random Matrix eigenvalue problems in structural dynamics," Int. J. Numer. Meth. Engng ; 69:562-591, 2007.

S. Adhikari, "Joint statistics of natural frequencies of stochastic dynamic systems," Comput. mech., 40:739-752, 2007.

M. Modares, R.L. Mullen and R.L. Muhanna, "Natural Frequencies of a Structure with Bounded Uncertainty," Journal of Engineering Mechanics, Vol. 132, No. 12, December 1, 2006.

W. Gao, "Natural frequency and mode shape analysis of structures with uncertainty," Mechanical systems and signal processing, 21, 24-39, 2007. 
J. Sim, Z.Qiu, X.Wang, "Modal analysis of structures with uncertain-but-bounded parameters via interval analysis," Journal of sound and vibration, 303, 29-45, 2007.

K. Sobczyk and J.Trebicki, "Maximum entropy principle in stochastic dynamics," Probabilistic Engineering Mechanics, Vol. 5, No. 3, 1990.

C.Soize, "Transient responses of dynamical systems with random uncertainties," Probabilistic Engineering Mechanics, Vol. 16, Issue 4, 363-372, 2001.

R.A.Livingston and J. Shuang, "Application of a maximum entropy method to estimate the probability density function of nonlinear or chaotic behavior in structural health monitoring data", Proceedings of the SPIE - The International Society for Optical Engineering, v 5765, n 1, p 749-57, 2005.

M.P.Mignolet and C.Soize, "Nonparametric stochastic modeling of linear systems with prescribed variance of several natural frequencies," Probabilistic Engineering Mechanics, 23, 267-278, 2008.

C.W. Hamilton and A.H.Hadijan, "Probabilistic frequency variations of structure-soil systems," Nuclear Engineering and Design, Vol 38, 303-322, 1976.

J.F.Clinton, S.C.Bradford, T.H.Heaton and J.Favela, "The observed wander of the natural frequencies in a structure", Bulletin of the Seismological Society of America, Vol. 96, No. 1, 237-257, February 2006.

R.Serfling, "Multivariate symmetry and asymmetry," Encyclopedia of Statistical Sciences, Second Edition (S. Kotz, N. Balakrishnan, C. B. Read and B. Vidakovic, eds.), pp. 53385345. Wiley, 2006 
G.Kimeldorf and A. Sampson, "Uniform representations of bivariate distributions," Communications in Statistics, v 4, n 7, 617-27, 1975. 


\section{APPENDICES}


APPENDIX - A

PROBABILITY DENSITY FUNCTIONS OF NATURAL

FREQUENCIES OF A SIMPLY SUPPORTED BEAM WITH SHEAR

DEFORMATIONS

Consider a simply-supported beam of length $L$ and mass per unit length, $m$. The beam has a uniform cross-section of area $A$ and the moment of inertia $I$. The Young's modulus and the shear modulus of the material of the beam are $E$ and $G$ respectively. The flexural rigidity of the beam is $E I$ and the shear rigidity is $G A$. The shape factor of the cross section is $k$ '.

The equation of motion for free transverse vibrations, $u(x, t)$ of the beam including the effect of shear deformation is given by

$$
m \frac{\partial^{2} u}{\partial t^{2}}-\left(\frac{E I \cdot m}{k^{\prime} \cdot G A}\right) \cdot \frac{\partial^{4} u}{\partial x^{2} t^{2}}+E I \frac{\partial^{4} u}{\partial x^{4}}=0
$$

The solution for the above equation is of the form:

$$
u(x, t)=f(x) \cdot g(t)
$$

Substituting Equation (A.2) in (A.1),

$$
\begin{gathered}
m f(x) \cdot \frac{\partial^{2} g(t)}{\partial t^{2}}-\left(\frac{E I \cdot m}{k^{\prime} \cdot G A}\right) \cdot \frac{\partial^{2} f(x)}{\partial x^{2}} \frac{\partial^{2} g(t)}{\partial t^{2}}+E I \cdot g(t) \frac{\partial^{4} f(x)}{\partial x^{4}}=0 \\
E I . g(t) \frac{\partial^{4} f(x)}{\partial x^{4}}=-\left[-\left(\frac{E I \cdot m}{k^{\prime} \cdot G A}\right) \cdot \frac{\partial^{2} f(x)}{\partial x^{2}}+m f(x)\right] \frac{\partial^{2} g(t)}{\partial t^{2}}
\end{gathered}
$$




$$
\frac{E I \cdot \frac{\partial^{4} f(x)}{\partial x^{4}}}{m\left[-\left(\frac{E I}{k^{\prime} \cdot G A}\right) \cdot \frac{\partial^{2} f(x)}{\partial x^{2}}+f(x)\right]}=-\frac{1}{g(t)} \cdot \frac{\partial^{2} g(t)}{\partial t^{2}}=\omega^{2}(\text { say })
$$

From Equation (A.5),

$$
\frac{\partial^{2} g(t)}{\partial t^{2}}+\omega^{2} \cdot g(t)=0
$$

The solution for $g(t)$ is of the form:

$$
g(t)=A \sin (\omega t+\psi)
$$

The solution for $f(x)$ from Equation (A.5)

$$
\begin{gathered}
\frac{E I \cdot \frac{\partial^{4} f(x)}{\partial x^{4}}}{m\left[-\left(\frac{E I}{k^{\prime} \cdot G A}\right) \cdot \frac{\partial^{2} f(x)}{\partial x^{2}}+f(x)\right]}=\omega^{2} \\
\frac{\partial^{4} f(x)}{\partial x^{4}}=\frac{m \omega^{2}}{E I}\left[-\left(\frac{E I}{k^{\prime} \cdot G A}\right) \cdot \frac{\partial^{2} f(x)}{\partial x^{2}}+f(x)\right]
\end{gathered}
$$

Let $\frac{m \omega^{2}}{E I}=\beta^{4}$. Then, the Equation (A.9) transforms to

$$
\frac{\partial^{4} f(x)}{\partial x^{4}}=\beta^{4}\left[-\left(\frac{E I}{k^{\prime} \cdot G A}\right) \cdot \frac{\partial^{2} f(x)}{\partial x^{2}}+f(x)\right]
$$

If $f(x)$ is of the form $f(x)=A e^{\alpha . x}$, Equation (A.10) gives

$$
\alpha^{4}=\beta^{4}\left[-\left(\frac{E I}{k^{\prime} \cdot G A}\right) \cdot \alpha^{2}+1\right]
$$


Equation (A.11) is a quadratic in $\alpha^{2}$ and can be solved in terms of $\beta$. The generalized solution for $f(x)$ is of the form:

$$
\begin{gathered}
f(x)=D_{1} \cosh \left(c_{1} \cdot x\right)+D_{2} \sinh \left(c_{1} \cdot x\right)+D_{3} \cosh \left(c_{2} \cdot x\right) \\
+D_{4} \sinh \left(c_{2} \cdot x\right)
\end{gathered}
$$

where $c_{1}$ and $c_{2}$ are constants.

Boundary conditions for a simply-supported beam are

$$
\left.\begin{array}{c}
f(x)=0 \\
\text { EI. } \frac{\partial^{2} f(x)}{\partial x^{2}}=0
\end{array}\right\} x=0 \text { and } x=L
$$

Applying the boundary conditions in Equation (A.13) to Equation (A.12), it can be found that the non-trivial solution (or eigen function) for $f(x)$ is of the form:

$$
f(x)=D \sin \left(\frac{n \pi}{L} \cdot x\right)
$$

From Equations (A.10) and (A.13),

$$
\begin{aligned}
& \left(\frac{n \pi}{L}\right)^{4}=\beta^{4}\left[-\left(\frac{E I}{k^{\prime} \cdot G A}\right) \cdot\left(\frac{n \pi}{L}\right)^{2}+1\right] \\
& \beta^{4}=\frac{\left(\frac{n \pi}{L}\right)^{4}}{\left[-\left(\frac{E I}{k^{\prime} \cdot G A}\right) \cdot\left(\frac{n \pi}{L}\right)^{2}+1\right]}=\frac{m \omega^{2}}{E I}
\end{aligned}
$$




$$
\begin{aligned}
& \omega^{2}=\frac{E I\left(\frac{n \pi}{L}\right)^{4}}{m\left[-\left(\frac{E I}{k^{\prime} \cdot G A}\right) \cdot\left(\frac{n \pi}{L}\right)^{2}+1\right]} \\
& \omega=\frac{n^{2} \pi^{2}}{\sqrt{m L^{4}}} \frac{\sqrt{E I} \sqrt{k^{\prime} G A}}{\sqrt{k^{\prime} G A+\left(\frac{n \pi}{L}\right)^{2} E I}}
\end{aligned}
$$

For a beam with predominant shear deformations, the simply supported beam is rigid in flexure i.e. $E I \rightarrow \infty$. So, the Equation (A.17) simplifies to

$$
\lim _{E I \rightarrow \infty} \omega_{n}=\frac{n^{2} \pi^{2}}{\sqrt{m L^{4}}} \sqrt{k^{\prime} G A}
$$

Equation (A.17) is similar to Equation (2.3) in Chapter - 2. Following steps identical to Equations (2.3) - (2.6), we can obtain the probability density function of the natural frequencies of the simply-supported shear beam given the distribution of shear rigidity, $G A$ $f_{G}(g)$.

$$
f_{\Omega_{n}}\left(\omega_{n}\right)=\frac{2 \omega_{n}}{B_{n}{ }^{2}} f_{G}\left(g=\frac{\omega_{n}{ }^{2}}{B_{n}{ }^{2}}\right) \text { where } B_{n}=\frac{n^{2} \pi^{2}}{\sqrt{m L^{4}}}
$$




\section{APPENDIX - B \\ DESIGN SPECTRA}

Monte Carlo simulation with multiple response spectrum analyses requires the earthquake input be characterized by a single input design spectrum. As discussed in chapter 4 , these spectra are generated corresponding to a non-exceedence probability of 0.84 for various damping ratios using the response spectrum of each of the 75 real time histories normalized to a unit value of PGA. The details of the earthquake records used are given in Table B.1. The design spectra are shown in Figure B.1

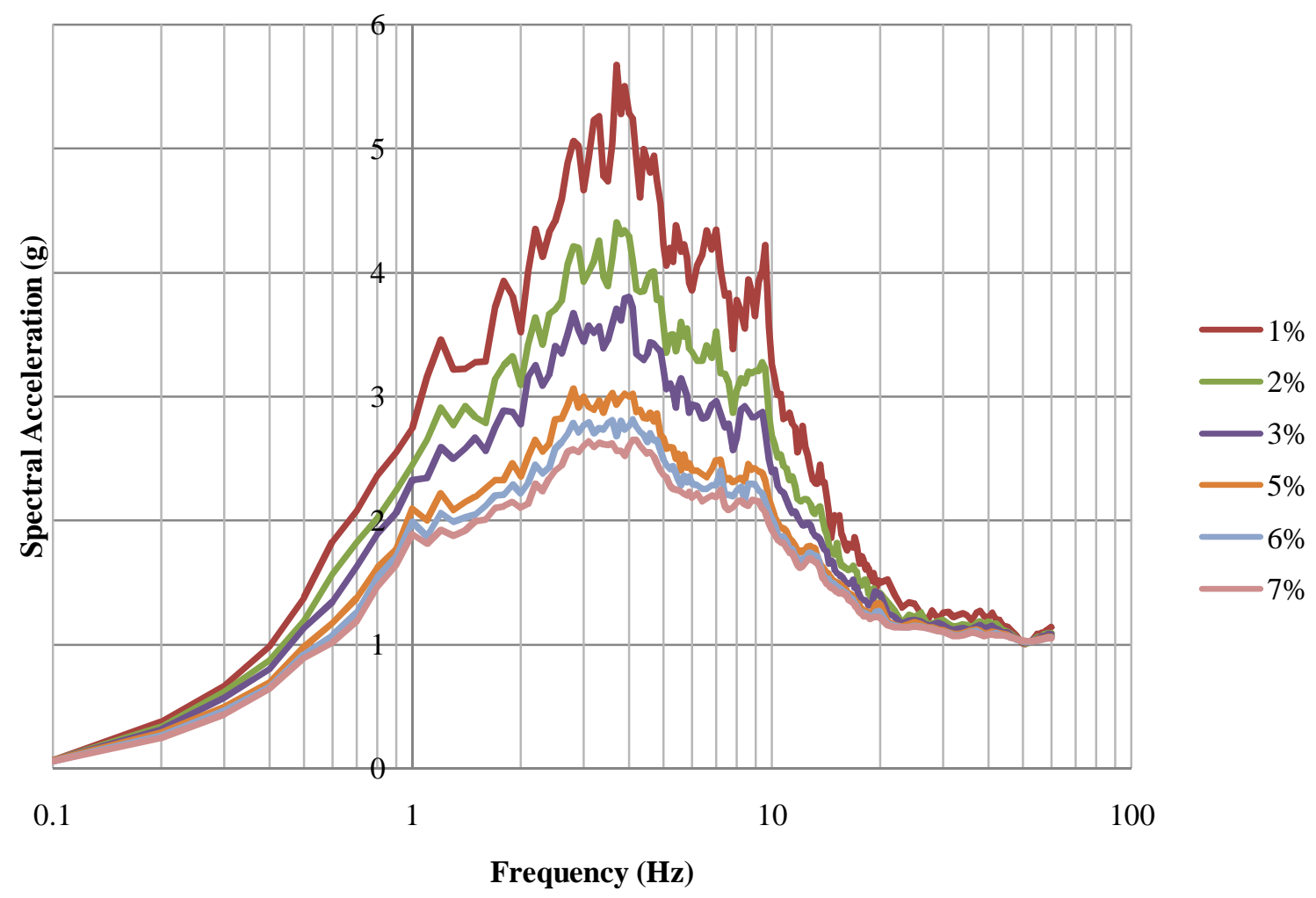

Figure B.1: Input spectrum corresponding to a non-exceedence probability of 0.84 for 75 real earthquake records 
Table B.1: List of real earthquake records

\begin{tabular}{|c|c|c|}
\hline \multirow{2}{*}{ Earthquake, site, date } & \multicolumn{2}{|c|}{ Component } \\
\hline & Record 1 & Record 2 \\
\hline Imperial Valley, Elcentro, 18, May 1940 & S00E & S90W \\
\hline Kern County, Pasadena, Caltech-Athenaeum, 21, July 1952 & S00E & S90W \\
\hline Kern County, Taft, Lincoln School Tunnel, 21, July 1952 & N21E & S69E \\
\hline Kern County, Santa Barbara, Court House, 21, July 1952 & N42E & S48E \\
\hline Kern County, Hollywood Storage, Basement, 21, July 1952 & S00W & N90E \\
\hline San Francisco, Golden Gate Park, 22, March 1957 & $\mathrm{~N} 10 \mathrm{E}$ & $\mathrm{S} 80 \mathrm{E}$ \\
\hline Long Beach, Vernon CMD Building, 10, March 1933 & S08W & N82W \\
\hline Lower California, ElCentro, Imperial Valley, 30, December 1934 & S00W & S90W \\
\hline Helena, Montana, Carrol Collage, 31, October 1935 & S00W & S90W \\
\hline Seattle, Distr. Engs. Office, 13, April 1949 & S02W & N88W \\
\hline Olympia, Hwy. Test Lab, 13, April 1949 & S04W & N86E \\
\hline Puget Sound, Olympia, Hwy. Test Lab, 29, April 1965 & S04W & N86E \\
\hline Parkfield, CA, Cholame, Shandon Array No. 2; 27, June 1966 & N65E & - \\
\hline Parkfield, Cholame, Shandon Array No. 5; 27, June 1966 & N05W & N85E \\
\hline Parkfield, Cholame, Shandon Array No. 8; 27, June 1966 & N50E & N40W \\
\hline Parkfield, Cholame, Shandon Array No. 12; 27, June 1966 & $\mathrm{~N} 50 \mathrm{E}$ & N40W \\
\hline Parkfield, Temblor, California No. 2; 27, June 1966 & N65W & S25W \\
\hline San Fernando, Pacoima Dam, 9, February 1971 & $\mathrm{~S} 16 \mathrm{E}$ & S74W \\
\hline San Fernando, 8244 Orion Blvd., $1^{\text {st }}$ Floor, 9, February 1971 & N00W & S90W \\
\hline San Fernando250 E. First St., Basement, 9, February 1971 & $\mathrm{~N} 36 \mathrm{E}$ & N54W \\
\hline San Fernando, 445 Figueroa St., Sub Basement, 9, February 1971 & N52W & S38W \\
\hline San Fernando, Hollywood, Storage, Basement, 9, February 1971 & S00W & N90E \\
\hline San Fernando, Caltec, Seismological Lab, 9, February 1971 & S00W & S90W \\
\hline San Fernando, Caltech-Athenaeum, 9, February 1971 & N00E & N90W \\
\hline San Fernando, Caltech Millikan Lib., Basement, 9, February 1971 & N00E & N90E \\
\hline San Fernando, Jet Propulsion Lab, Basement, 9, February 1971 & S82E & S08W \\
\hline San Fernando, Fire Station, Storage Room, 9, February 1971 & S60E & S30W \\
\hline San Fernando, 15250 Ventura Blvd., Basement, 9, February 1971 & N11E & N79W \\
\hline Landers, Lucerne Valley, Station, 28, June 1992 & N15W & N80W \\
\hline Northridge, Pardee Station, 17, January 1994 & S00E & N90E \\
\hline Northridge, Rinaldi, Receiving, Station, 17, January 1994 & $\mathrm{~N} 42 \mathrm{~W}$ & S48W \\
\hline Northridge, Sylmar, onverter, Station, 17, January 1994 & N52E & S38E \\
\hline Northridge, Sylmar, onverter, Station East, 17, January 1994 & $\mathrm{~N} 18 \mathrm{E}$ & N72W \\
\hline Northridge, Newhall-LA, County, Fire Station, 17, January 1994 & N90E & N00E \\
\hline Northridge, Arleta Fire, Station, 17, January 1994 & N90E & N00E \\
\hline Northridge, Tarzana-Cedar, Hill, Nuresery, 17, January 1994 & N90E & N00E \\
\hline Northridge, Sylmar-county, Hospital, 17, January 1994 & N90E & N00E \\
\hline
\end{tabular}


Table B.1 (Continued)

\begin{tabular}{|l|l|l|}
\hline \multicolumn{1}{|c|}{ Earthquake, site, date } & \multicolumn{2}{c|}{ Component } \\
\cline { 2 - 3 } & Record 1 & Record 2 \\
\hline Northridge, Santa Monica, City Hall, 17, January 1994 & N90E & N00E \\
\hline
\end{tabular}

